\title{
Situational Awareness Enhancement for Connected and Automated Vehicle Systems
}

Amin Tahmasbi Sarvestani

Follow this and additional works at: https://researchrepository.wvu.edu/etd

\section{Recommended Citation}

Tahmasbi Sarvestani, Amin, "Situational Awareness Enhancement for Connected and Automated Vehicle Systems" (2016). Graduate Theses, Dissertations, and Problem Reports. 6766.

https://researchrepository.wvu.edu/etd/6766

This Dissertation is protected by copyright and/or related rights. It has been brought to you by the The Research Repository @ WVU with permission from the rights-holder(s). You are free to use this Dissertation in any way that is permitted by the copyright and related rights legislation that applies to your use. For other uses you must obtain permission from the rights-holder(s) directly, unless additional rights are indicated by a Creative Commons license in the record and/ or on the work itself. This Dissertation has been accepted for inclusion in WVU Graduate Theses, Dissertations, and Problem Reports collection by an authorized administrator of The Research Repository @ WVU.

For more information, please contact researchrepository@mail.wvu.edu. 


\title{
Situational Awareness Enhancement for Connected and Automated Vehicle Systems
}

\author{
Amin Tahmasbi Sarvestani \\ Dissertation submitted to the \\ College of Engineering and Mineral Resources \\ at West Virginia University \\ in partial fulfillment of requirements for the degree of \\ Doctor of Philosophy in \\ Computer Science
}

Yaser P. Fallah, Ph.D., Chair

Parviz Famouri, Ph.D.

Elaine M. Eschen, Ph.D.

Vinod K. Kulathumani, Ph.D.

Jason N. Gross, Ph.D.

Department of Computer Science and Electrical Engineering

\author{
Morgantown, West Virginia \\ 2016
}

Keywords: Connected and Automated Vehicles, CAV, ITS, V2V, V2P, Situational A wareness

Copyright 2016 Amin Tahmasbi Sarvestani 


\section{ABSTRACT \\ Situational Awareness Enhancement for Connected and Automated
Vehicle Systems}

Amin Tahmasbi Sarvestani

Recent developments in the area of Connected and Automated Vehicles (CAVs) have boosted the interest in Intelligent Transportation Systems (ITSs). While ITS is intended to resolve and mitigate serious traffic issues such as passenger and pedestrian fatalities, accidents, and traffic congestion; these goals are only achievable by vehicles that are fully aware of their situation and surroundings in real-time. Therefore, connected and automated vehicle systems heavily rely on communication technologies to create a real-time map of their surrounding environment and extend their range of situational awareness. In this dissertation, we propose novel approaches to enhance situational awareness, its applications, and effective sharing of information among vehicles.

The communication technology for CAVs is known as vehicle-to-everything (V2x) communication, in which vehicle-to-vehicle (V2V) and vehicle-to-infrastructure (V2I) have been targeted for the first round of deployment based on dedicated short-range communication (DSRC) devices for vehicles and road-side transportation infrastructures. Wireless communication among these entities creates self-organizing networks, known as Vehicular Ad-hoc Networks (VANETs). Due to the mobile, rapidly changing, and intrinsically error-prone nature of VANETs, traditional network architectures are generally unsatisfactory to address VANETs fundamental performance requirements. Therefore, we first investigate imperfections of the vehicular communication channel and propose a new modeling scheme for large-scale and small-scale components of the communication channel in dense vehicular networks. Subsequently, we introduce an innovative method for a joint modeling of the situational awareness and networking components of CAVs in a single framework. Based on these two models, we propose a novel network-aware broadcast protocol for fast broadcasting of information over multiple hops to extend the range of situational awareness. Afterward, motivated by the most common and injury-prone pedestrian crash scenarios, we extend our work by proposing an end-to-end Vehicle-to-Pedestrian (V2P) framework to provide situational awareness and hazard detection for vulnerable road users. Finally, as humans are the most spontaneous and influential entity for transportation systems, we design a learning-based driver behavior model and integrate it into our situational awareness component. Consequently, higher accuracy of situational awareness and overall system performance are achieved by exchange of more useful information. 


\section{Dedication}

This dissertation is dedicated to my brilliant and outrageously loving and supportive wife,

Hoda Nikpour,

and to my always encouraging, ever faithful parents,

Shokat and Aliasghar Tahmasbi Sarvestani. 


\section{Acknowledgments}

Foremost, I would like to express my sincere gratitude to my advisor Dr. Yaser P. Fallah for the continuous support of my $\mathrm{Ph} . \mathrm{D}$. study and related research, for his patience, motivation, and immense knowledge. His guidance helped me in all the time of research and writing of this dissertation. I could not have imagined having a better advisor and mentor for my Ph.D. study.

Besides my advisor, I would like to thank the rest of my thesis committee: Prof. Parviz Famouri, Dr. Elaine M. Eschen, Dr. Vinod K. Kulathumani, and Dr. Jason N. Gross, for their encouragement, insightful comments, and hard questions which incented me to widen my research from various perspectives.

My sincere thanks also go to Dr. Mohammad Naserian and Dr. Hariharan Krishnan, for offering me the summer internship opportunities in their groups and leading me working on diverse exciting projects.

I thank my fellow labmates in Cyber-physical system group: Ehsan Moradi-Pari, S M Osman Gani, Neda Nasiriani, Hossein Nourkhiz Mahjoub, Hadi Kazemi, Chanukya Gadde, Mohammad Fanaei, and Mehdi Iranmanesh for the stimulating discussions, for the sleepless nights we were working together before deadlines, and for all the fun we have had in the last four and a half years. Also, I thank all of my friends at West Virginia University.

Last but not the least, I would like to thank my family: my wife, my parents, and my sister for supporting me spiritually throughout my Ph.D. study and my life in general. 


\section{TABLE OF CONTENTS}

Chapter

Page

DEDICATION

CHAPTER 2 SITUATIONAL AWARENESS BEYOND LINE-OF-SIGHT THROUGH VEHICULAR COMMUNICATION ...19

2.1 Composite ChANNel Modeling of VANETS Using LARGE RSSI MeASUREMENT .....................................................19

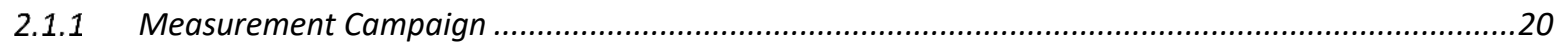

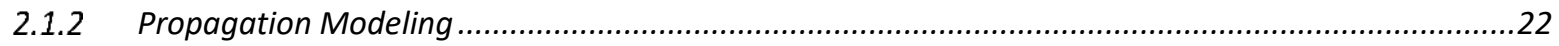

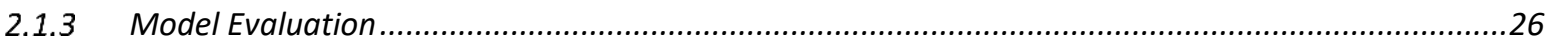

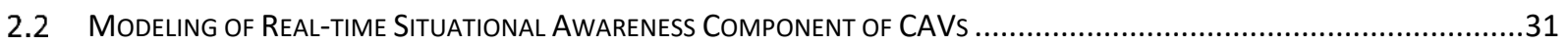

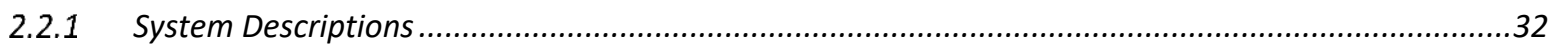

2.2.2 Model Description for the Fully Connected Network …...............................................................34

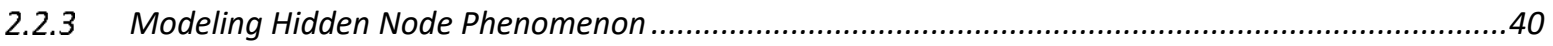

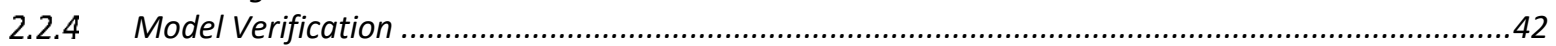

CHAPTER 3 MULTI-HOP SITUATIONAL AWARENESS THROUGH NETWORK-AWARE DOUBLE-LAYER DISTANCE-

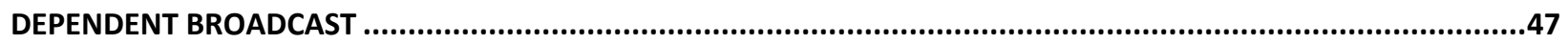

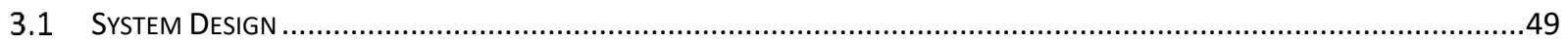

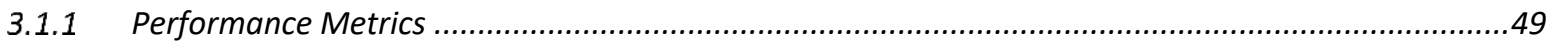

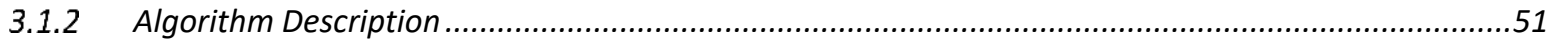

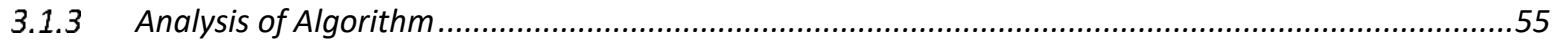

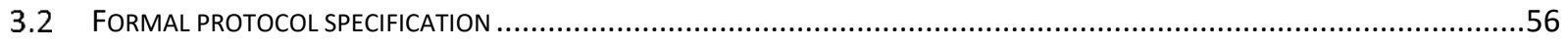

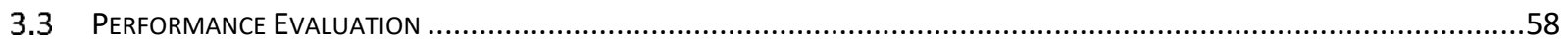

CHAPTER 4 SITUATIONAL AWARENESS AND HAZARD DETECTION FOR VULNERABLE ROAD USERS.................64

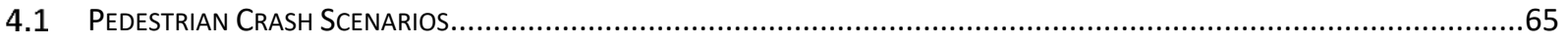

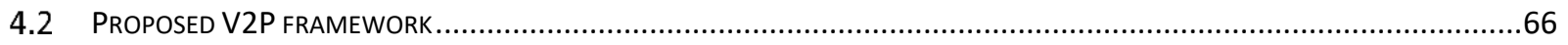

4.2.1 Target Classification and Collision Detection Algorithms ..........................................................69

4.2.2 Congestion Control and Power Consumption Control Mechanisms.................................................71

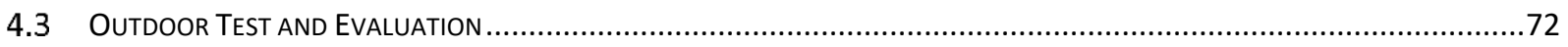

CHAPTER 5 SITUATIONAL AWARENESS ENHANCEMENT WITH LEARNING-BASED DRIVER BEHAVIOR MODELING 79 


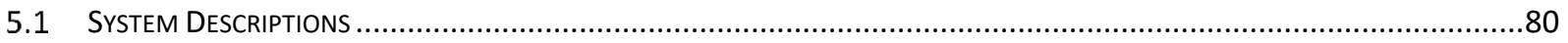

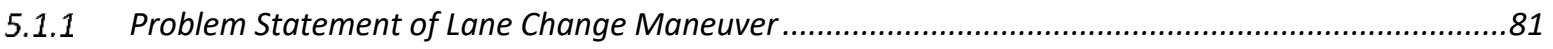

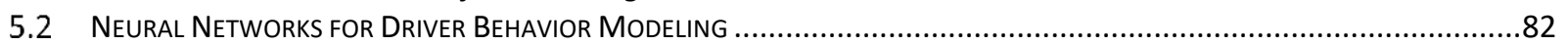

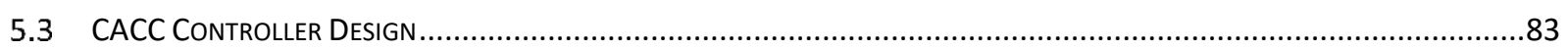

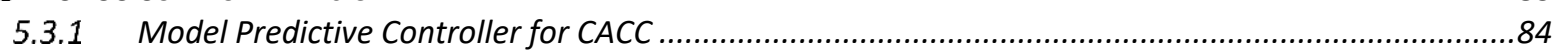

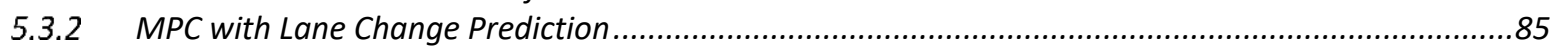

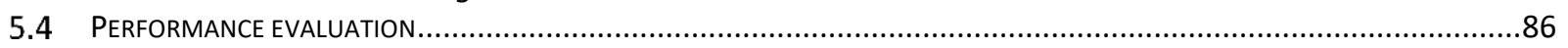

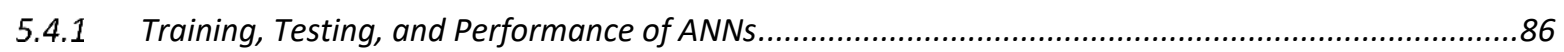

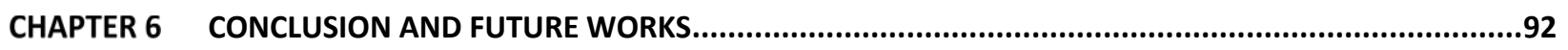

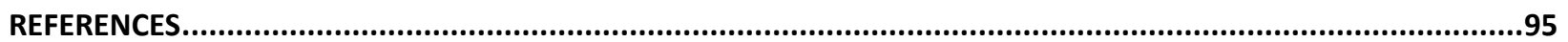




\section{LIST OF TABLES}

Table

Page

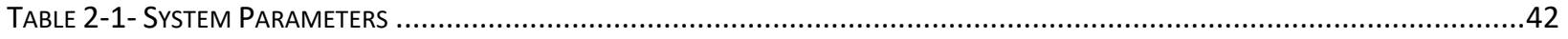

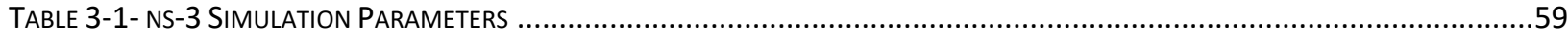

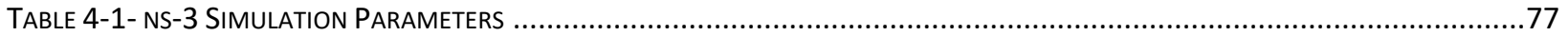




\section{LIST OF FIGURES}

Figure

Page

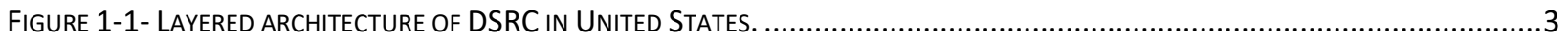

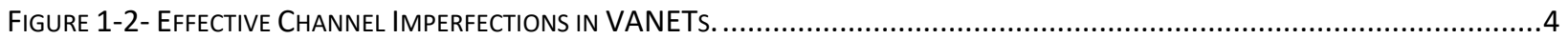

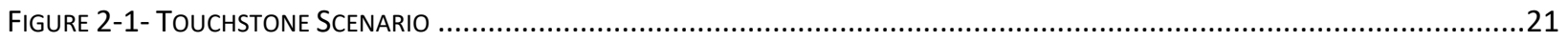

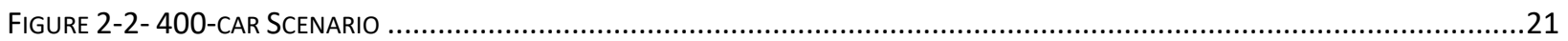

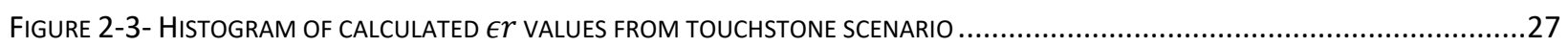

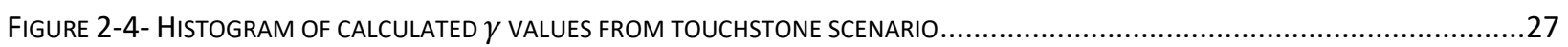

FIGURE 2-5-VALIDATION OF THE PROPOSED APPROACH USING ONE-METER BIN AVERAGED RSSI VALUES OF TOUCHSTONE SCENARIO .....28

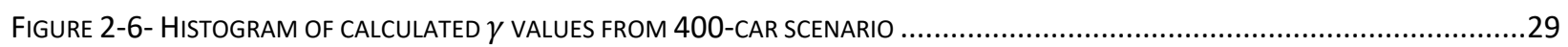

FIGURE 2-7- BOX PLOT OF EMPIRICAL DATA (BLUE) VERSUS REGENERATED POINTS FROM $\alpha-\mu$ DISTRIBUTION (RED) IN 400-CAR

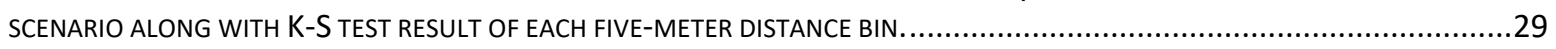

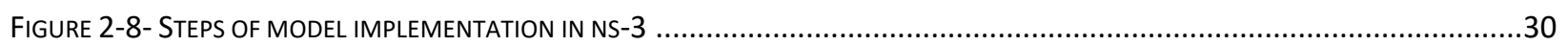

FIGURE 2-9- BOX PLOT OF EMPIRICAL DATA (BLUE) VERSUS NS-3 SIMULATION RESULTS (RED) IN 400-CAR SCENARIO...........................31

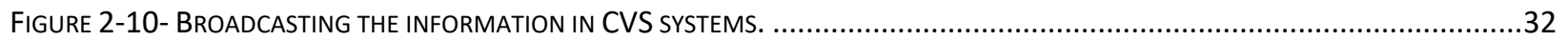

FIGURE 2-11- TWO DIFFERENT COMPONENTS OF REAL-TIME SITUATIONAL AWARENESS. .......................................................33

FigURE 2-12- PRoBABILISTIC TIMED-AUTOMATA MODEL FOR SENDER AT VEHICLE $j$ IN THE SATURATION CONDITION.............................35

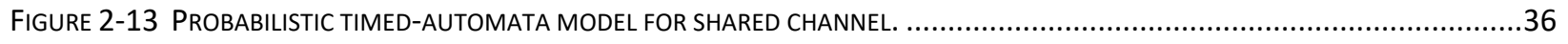

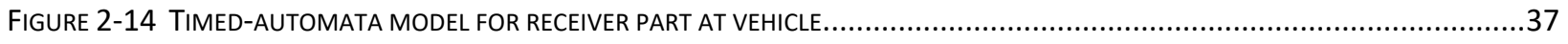

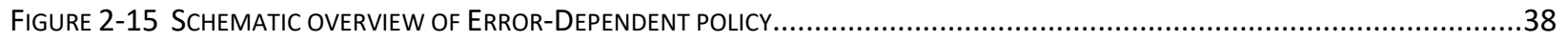

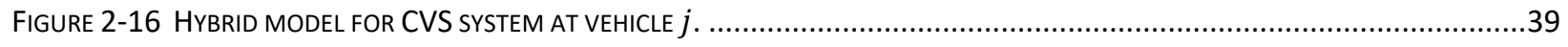

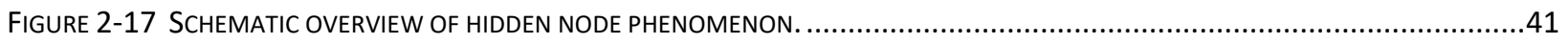

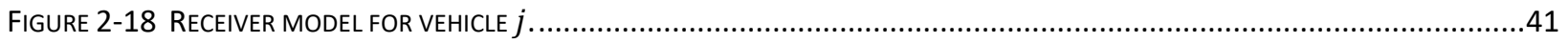

FIGURE 2-19 PROBABILITY OF SUCCESS VS. NUMBER OF VEHICLES - ALL IN RANGE SCENARIO .....................................................43

FIGURE 2-20 PROBABILITY OF SUCCESS VS. TRANSMISSION RATE - ALL IN RANGE SCENARIO. .....................................................44

FIGURE 2-21 PROBABILITY OF SUCCESS VS. TRANSMISSION RANGE - HIDDEN NODE SCENARIO.................................................45

FIGURE 2-22 PROBABILITY OF SUCCESS VS. TRANSMISSION RATE AND RANGE - HIDDEN NODE SCENARIO.......................................45

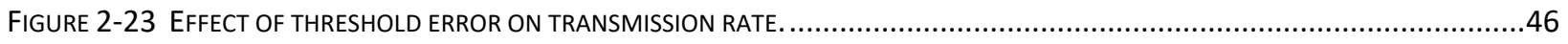

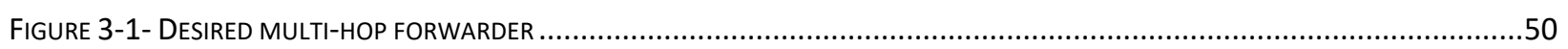

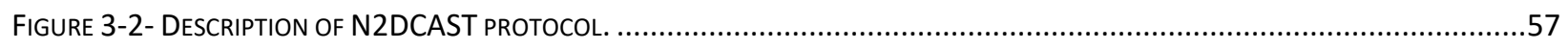

FIGURE 3-3- PER VERSUS DISTANCE FOR CONSTANT SPEED AND SUMO SCENARIOS WITH TWO DIFFERENT VEHICLE DENSITIES FOR FIX-

RANGE CHANNEL PROPAGATION MODEL. ALL SCENARIOS, EXCEPT THE ONE NOTED, HAVE MESSAGE SIZE CONTROL. ....................60

FIGURE 3-4- PER VERSUS DISTANCE FOR CONSTANT SPEED AND SUMO SCENARIOS WITH THREE-LOG CHANNEL PROPAGATION MODEL AND

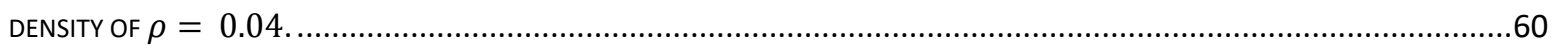

FIGURE 3-5- SUCCESS PROBABILITY OF REBROADCAST VERSUS DISTANCE FOR CONSTANT SPEED AND SUMO SCENARIOS, TWO VEHICLE DENSITIES, AND TWO PROPAGATION MODELS. THE CURVE OF ISPF (EQUATION (3.8)) VERSUS DISTANCE WITH PARAMETER $\lambda i=$ 3.8 AND UNIFORM PROBABILISTIC METHOD WITH PARAMETER $\delta=10$ ARE ALSO SHOWN..............................................61

FIGURE 3-6- AVERAGE NUMBER OF TDUS PER BEACON FOR EACH VEHICLE ALONG THE ROAD FOR DIFFERENT REBROADCAST METHODS. THE FORWARDER RATIO, WHICH IS AVERAGED OVER ALL VEHICLES, IS 0.9984, 0.9958, AND 1.012 FOR PROBABILISTIC, COUNTERBASED, AND N2DCAST METHODS, RESPECTIVELY.

FIGURE 3-7- STALENESS FOR EACH HOP VERSUS DISTANCE FOR CONSTANT SPEED AND SUMO SCENARIOS, TWO VEHICLE DENSITIES, AND FIX-RANGE PROPAGATION MODEL. AlL SCENARIOS, EXCEPT THE ONE NOTED, HAVE MESSAGE SIZE CONTROL............................62

FIGURE 3-8- STALENESS VERSUS DISTANCE FOR CONSTANT SPEED AND SUMO SCENARIOS WITH THREE-LOG PROPAGATION AND VEHICLE

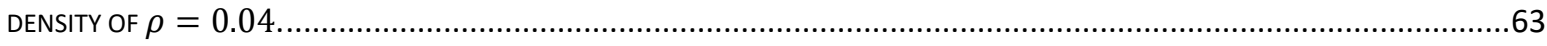

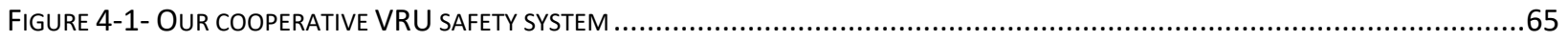

FIGURE 4-2- THE MOST COMMON AND INJURY-PRONE VEHICLE-PEDESTRIAN CRASH SCENARIOS .................................................66

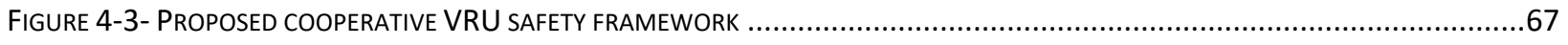

FIGURE 4-4- DIFFERENT SEVERITIES OF POSSIBLE CRASHES IN STRAIGHT AND CURVED PATHS ...................................................69 
FIGURE 4-5- PROOF-OF-CONCEPT TEST OF OUR COOPERATIVE VRU SAFETY FRAMEWORK..................................................73

FIGURE 4-6- RELATIVE PATH OF THE SMARTPHONE FROM THE VEHICLE PERSPECTIVE. ....................................................

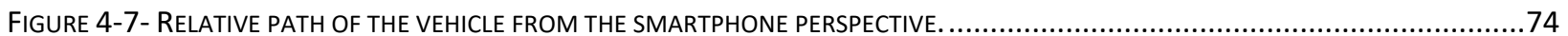

FIGURE 4-8- CORRESPONDING DISTANCE-TO-STOP OF EACH ZONE ALONG WITH THE LONGITUDINAL DISTANCE OF VEHICLE AND

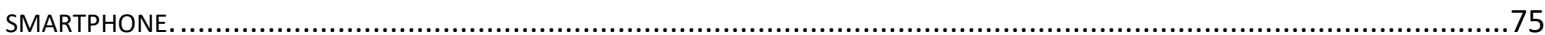

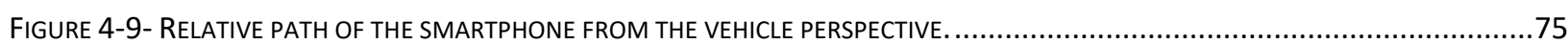

FIGURE 4-10- RELATIVE PATH OF THE VEHICLE FROM THE SMARTPHONE PERSPECTIVE. ........................................................76

FIGURE 4-11- CORRESPONDING DISTANCE-TO-STOP OF EACH ZONE ALONG WITH THE LONGITUDINAL DISTANCE OF VEHICLE AND SMARTPHONE.

FIGURE 4-12- CONGESTION CONTROL PERFORMANCE IN TERMS OF PER VERSUS DISTANCE....................................................77

FIGURE 4-13- AVERAGED CBP VERSUS TIME FOR WITH AND WITHOUT CONGESTION CONTROL SCENARIOS.....................................78

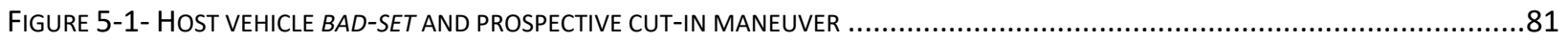

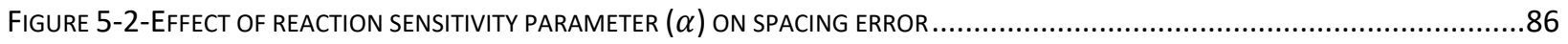

Figure 5-3- SMOOTHED, NoRMALIZED, AND INTEGRATED INPUT SIGNALS OF A SINGLE LANE CHANGE MANEUVER ............................87

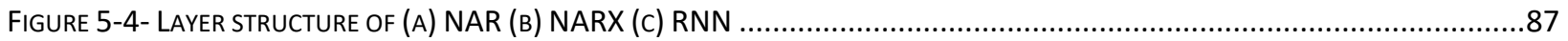

FIGURE 5-5- COMPARISON OF 90-PERCENTILE CONF. INTERVAL OF LATERAL POSITION PREDICTION OF THE KINEMATIC AND RNN MODELS

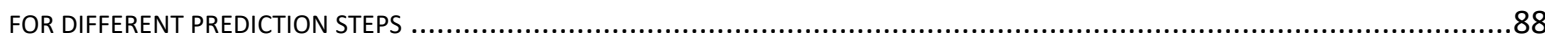

FIGURE 5-6- COMPARISON OF 90-PERCENTILE CONF. INTERVAL OF LONGITUDINAL POSITION PREDICTION OF THE KINEMATIC AND RNN

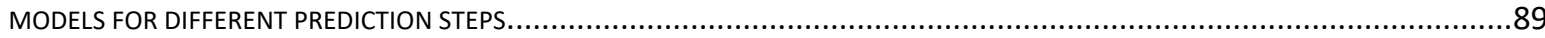

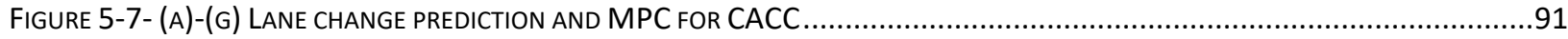




\section{Chapter 1 Introduction and Related Works}

Serious traffic problems such as accidents and traffic jams have been growing due to the increasing number of motorists in recent years. Therefore, new modifications and improvements are required for existing transportation systems. The Intelligent Transportation Systems (ITSs) are promising solutions to increase safety and efficiency which can integrate telecommunication, information, and cyber technologies into transportation systems. This integration enables the vehicles to be fully aware of their situation and surroundings in real-time. Therefore, these systems heavily rely on communication technologies, with low latency and high throughput, to create a real-time map of their surrounding environment and extend their range of situational awareness. Safety applications such as collision warnings and collision avoidance, which alert the driver and stop the vehicle in imminent accident scenarios, are the primary goals of ITS. Other applications such as road congestion information and electronic toll collection are also used for efficiency and traffic management purposes. Both of such ITS applications are built on top of connected and automated vehicle (CAV) systems linked by wireless communication technologies known as Vehicular Adhoc Networks (VANETs).

The wireless communication in VANETs is intrinsically error-prone, due to communication channel imperfections such as noise, path-loss, and interference. Moreover, high-speed vehicles rapidly change the VANET topologies which adds to the above issues. Therefore, traditional network architectures are generally unsatisfactory to address VANETs fundamental performance requirements such as throughput and latency. Consequently, unlike the traditional layered communication stack, in which interaction among layers is limited to delivering concealed information and happens only between neighboring layers, an information sharing scheme among layers is required in VANETs to effectively overcome aforementioned issues. This concept, which is introduced as a network-aware approach in VANETs, could attain higher throughput and lower latency by simultaneously tweaking and optimizing parameters of different layers. For example, the information acquired from physical (PHY) and medium access control (MAC) layers such as level of interference and number of dropped packets can strengthen network protocols to discover stable links. 
In this dissertation, we propose novel network-aware approaches to enhance real-time situational awareness, its applications, and effective sharing of information among vehicles. Vehicular communication provides real-time situational awareness beyond line-of-sight. Therefore, we first study the imperfections of the vehicular communication channel and propose a new modeling scheme for large-scale and small-scale components of the vehicular communication channel in dense vehicular networks. Subsequently, we introduce an innovative method for a joint modeling of the situational awareness and communication components of CAVs in a single framework to achieve real-time situational awareness beyond line-of-sight (LOS). Based on these two models, we propose a novel network-aware broadcast protocol for fast broadcasting of information over multiple hops to extend the range of situational awareness. Afterward, rooted on the most common and injury-prone pedestrian crash scenarios, we extend our work by proposing an end-to-end Vehicle-to-Pedestrian (V2P) framework to provide situational awareness and hazard detection for vulnerable road users. Finally, as humans are the most spontaneous and influential entity for transportation systems, we design a learning-based driver behavior model and integrate it into the estimation component of our situational awareness system. Consequently, higher accuracy of situational awareness and overall system performance are achieved by exchange of more useful information.

This chapter is divided into six sections. Section 1.1 reviews the layered architecture for VANETs. Section 1.2 discusses the existing works on situational awareness through wireless communication and VANETs. Literature review and introduction to network-aware multi-hop situational awareness are presented in section 1.3. Section 1.4 is dedicated to the network-aware situational awareness and hazard detection for vulnerable road users. Section 1.5 briefs the existing literature for driver behavior modeling in terms of lane change detection and vehicle trajectory prediction. Our contributions and organization of the dissertation are discussed in section 1.6.

\subsection{VANETs Layered Architecture}

Figure 1-1 shows the protocol stack of dedicated short range communication (DSRC), including names of protocols and standards defined for each layer [1]. Beneath these layers is the wireless channel, which is the communication medium for VANETs. The vehicle-to-vehicle (V2V) communication in the United States is founded on top of DSRC standards. IEEE 802.11p Wireless Access for Vehicular Environments (WAVE), which is a modification of the familiar IEEE 802.11 (Wi-Fi) standard, is used at the PHY and MAC layers. A group of standards defined by the IEEE 1609 Working Group is utilized in the middle of the DSRC stack: 1609.4 for Channel Switching, 1609.3 for Network Services (including the WAVE Short Message Protocol-WSMP), and 1609.2 for Security Services. Internet protocols for the Network and Transport layers, i.e., Internet Protocol version 6 (IPv6), User Datagram Protocol (UDP) and Transmission Control Protocol (TCP) are also supported by DSRC. The requirements of a given application define the choice between using WSMP or IPv6+UDP/TCP. Safety-related and single-hop messages typically utilize the bandwidth-efficient WSMP. 


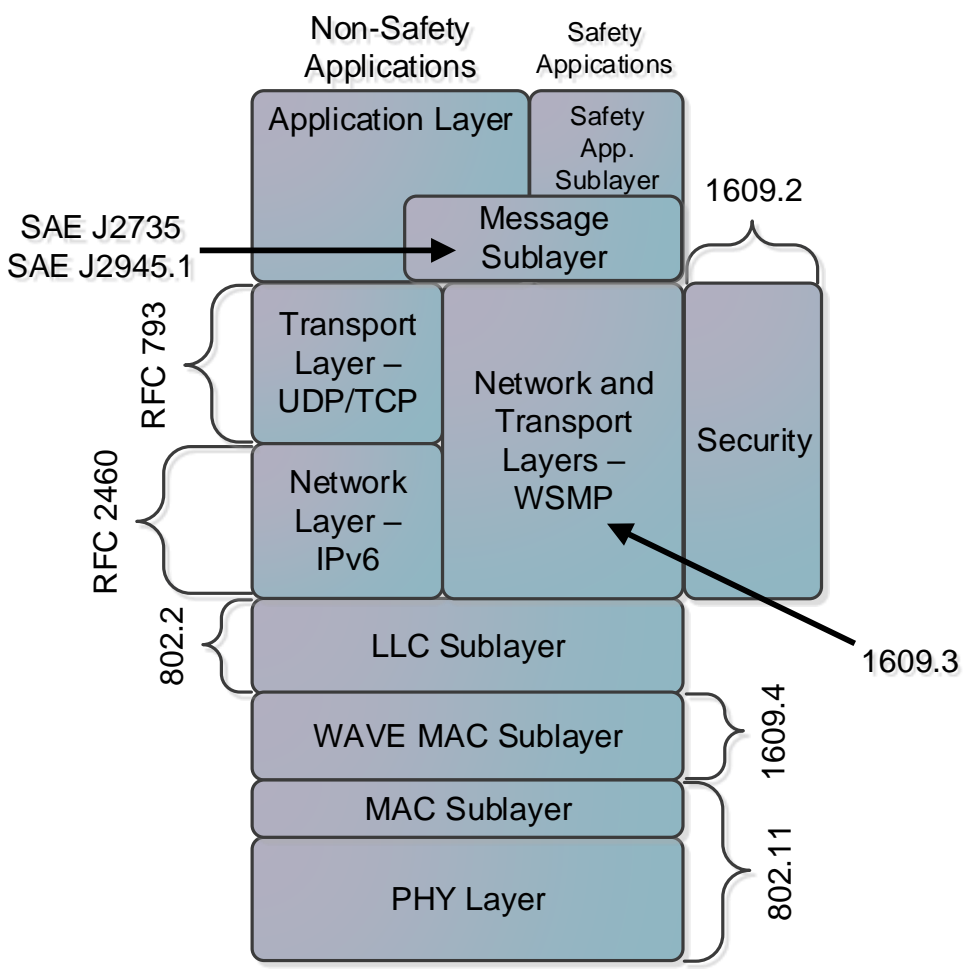

Figure 1-1- Layered architecture of DSRC in United States.

Application related standards are placed at the top of the stack. For example, the SAE J2735 Message Set Dictionary standard specifies a set of message formats that support vehicle-based applications. The most important format is the basic safety message (BSM), which carries critical vehicle state information in support of V2V safety applications. Using V2V applications, vehicles track the position and movement of each other to take action and prevent possible crashes by periodically exchanging BSMs.

\subsection{Situational Awareness beyond Line-Of-Sight through Vehicular Communication}

While VANETs can provide a beyond line-of-sight situational awareness, comprehension and characterization of their communication medium have a vital role in the architectural design of vehicular safety systems and optimization of their parameters. 


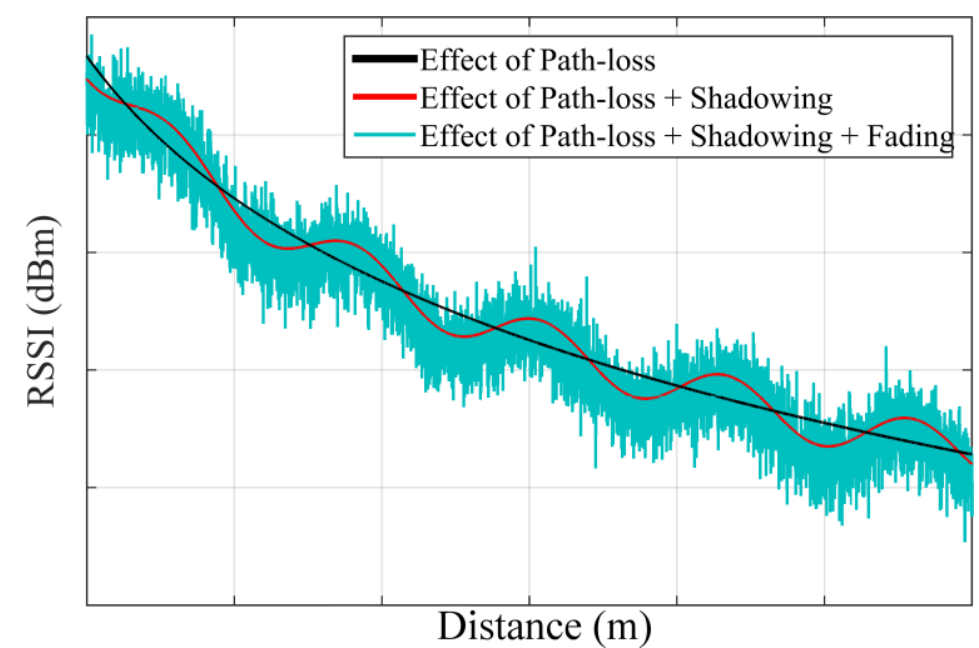

Figure 1-2- Effective Channel Imperfections in VANETs.

The main imperfections of vehicular channels in different vehicular scenarios are surveyed in [24] which can be categorized as (Figure 1-2)

- Path-loss is the large-scale deterministic propagation model which characterizes the amount of attenuation on the average signal power at the receiver, based on its distance from the transmitter.

- Shadowing or large-scale fading is a non-deterministic model which describes the variations in the received signal power over distances which are much greater than the signal wavelength. Shadowing mainly happens in non-line-of-sight (NLOS) scenarios due to obstruction and yields to strong attenuation and variation of signal strength. The dimensions of involved obstacles are considerably greater than the wavelength of the signal, such as buildings, trees, and hills.

- Small-scale fading is a stochastic representation of diffraction, reflection, and scattering effects on the transmitted signal. Their after-effect is multiple waves arriving from different directions to the antenna with various amplitudes and phases. It can be noted that diffraction, reflection, and scattering are consequences of transmitter or receiver movement.

Other imperfections such as power delay profile (PDP) and Doppler spread are also studied for vehicular channels which are out of the scope of this work [5].

Parameters of vehicular channel models are mainly derived from utilizing two main measurement methods: channel sounder devices and received signal strength indicator (RSSI). On one hand, although channel sounders are specifically designed to determine the frequency response of the channel, they can only be used in single transmitter-receiver scenarios; considering the fact that channel characterization of the situation of numerous transceivers is not feasible with channel sounders, as the experiment setup is complicated and very expensive. On the other hand, RSSI values can be extracted merely from the received packets with no extra cost or setup. Taking into 
account that single transmitter-receiver scenarios are not an appropriate representative of the reallife channels as each vehicle is not only a transceiver, but also an obstacle; therefore, RSSI-based methods may become more suitable for parameter identification of real-life channels with high number of vehicles, even though they are not primarily recorded to be used for channel modeling.

Different measurement campaigns have been established and numerous scenarios were investigated with respect to various surrounding environments of VANETs to identify the aforementioned parameters of vehicular channels based on both channel sounder devices and RSSI-based measurements.

Two different path-loss models are proposed for an inter-vehicle communication channel for three different environments: rural, highway, and urban [6]. Measurement framework was set up at 5.9 $\mathrm{GHz}$, using a channel sounder at the transmitter side which generated a multi-tone signal and one receiver. A two-ray model with a zero mean lognormal shadowing is derived as a rough path loss model for both rural and highway environments for distances larger than 32 meters. For urban scenario, a general log-distance model with a zero mean lognormal shadowing is recommended. The path-loss exponent of 1.61 is calculated based on their measured data set.

Path-loss, power delay profile, and delay-Doppler spectrum analysis in the highway, rural, urban and suburban environments are investigated using channel sounder [7-10]. Measurements were performed over the $240 \mathrm{MHz}$ band around $5.2 \mathrm{GHz}$ central frequency. Two GPS-equipped trucks were employed as transmitter and receiver. The most important characteristic of all of their measurement scenarios is the dominant line-of-sight (LOS) between transmitter and receiver. Logdistance model plus a zero mean lognormal shadowing is proposed for highway, urban, and suburban environments. The parameters are evaluated for the measured data using least mean square error curve fitting method. Due to the low density of scatterers, which leads to a dominant LOS plus a ground reflection, two-ray is considered to be the correct path-loss model in the rural environment. The validity of the models is claimed for distances greater than 10 and 20 meters for log-distance and two-ray, respectively.

In [11], fading statistics are analyzed in four environments: highway, motorway, urban and suburban. Two vehicles, one transmitter equipped with a $5.2 \mathrm{GHz}$ signal generator and one receiver equipped with a network analyzer were used for data collection. It is claimed that urban and suburban environments have the same behavior, in terms of small-scale fading, as motorway and highway scenarios, respectively. Rician, Rayleigh, Gaussian and Nakagami- $m$ distributions were examined and Rician channel model was found to be the best match in all scenarios based on the least mean absolute error method which was reasoned by dominant LOS in all measurements. Lognormal model was offered to be used for shadowing in all scenarios. It should be mentioned that flat fading assumption for vehicular channels is insupportable for DSRC-based networks [12, 13]. Therefore, due to frequency selective fading of DSRC channel, whereas RSSI values represent the effect of total channel attenuation through energy measurement, their use is best limited to path-loss, shadowing, and fading analysis [14].

Boban et al in [15-18] considered the intermediate vehicles between transmitter and receiver as dynamic obstacles and added them to static obstacles like buildings in the path-loss model calculations. The results indicate that path-loss of the vehicular channel, packet delivery ratio (PDR), latency, and jitter completely depend on the presence of LOS. Two vehicles, one 
transmitter, and one receiver were equipped with NEC LinkBird-MX which is a DSRC platform working based on IEEE 802.11p on $5.9 \mathrm{GHz}$ frequency. RSSI and PDR values were logged in different measurement scenarios. The results supported the two-ray as the best fitted path-loss model in the presence of LOS for highway, suburban, and urban areas.

Empirical RSSI values from a measurement campaign in freeway environment, divided into tenmeter bins, were used to find the best fitted fading distribution [14]. Nakagami- $m$ distribution is proposed as an appropriate fit based on cumulative distribution function (CDF) matching approach. Reported values of shape parameter $m$ for Nakagami- $m$ distribution lies between 1 to 1.8 for distances less than 100 meters, and 0.7 to 1 beyond it. Therefore, behavior of Nakagami- $m$ distribution is dominantly similar to Rician and Rayleigh for distances below and above 100 meters, respectively.

In another effort [19], path-loss and fading were analyzed for the suburban environment based on two RSSI data sets collected from two vehicles as a transmitter-receiver pair. A dual slope logdistance path-loss model plus a zero mean lognormal shadowing is suggested to model large-scale power attenuation. Different path-loss exponents and shadowing standard deviations for distances under and above 100 meters are calculated using regression methods. Small-scale fading is modeled using Nakagami- $m$ distribution. The first data set tends toward Rician and Rayleigh distributions for distances under and above 70 meters, respectively. The other data set has the same behavior pattern except that the change point is around 90 meters.

A similar approach is taken to derive the log-distance path-loss and zero mean log-normal shadowing parameters from RSSI measurements in highway and rural environments [20]. Due to the fewer number of obstacles, the critical distance for slope change in path-loss model is reported much closer to the first Fresnel zone. Moreover, shadowing standard deviations of rural and highway environments are also less than shadowing standard deviation of the suburban environment of [19].

RSSI-based measurements with a single transmitter-receiver pair of vehicles in motorway, rural and urban environments have been utilized to identify the best fading model for vehicular scenarios in [21]. Dual-slope log-distance path-loss with a zero mean log-normal shadowing and Nakagami$m$ fading models are derived as the best match for large- and small-scale received power variations, respectively.

Nakagami- $m$ distribution is also selected to model the small-scale fading of the vehicle-to-vehicle (V2V) channel based on the RSSI measurements in a highway and an open space area [22]. The results propose greater values of Nakagami- $m$ shape parameter $m$ for open space in comparison with highway.

A simulation based study of IEEE 802.11p also takes Nakagami- $m$ fading to model the vehicular networks channel [23]. A suburban scenario is simulated in OPNET with an updated physical layer model which has Nakagami- $m$ fading model included. They found the best value for shape parameter $m$, as a function of separation distance and relative velocity of transmitter and receiver.

In our work, we take a different approach with a large data set of RSSI values to derive path-loss parameters for the two-ray propagation model. Furthermore, we use $\alpha-\mu$ distribution, which is a 
more general fading model compared to Nakagami- $m$, to model the small-scale fading of the vehicular environment, while its goodness-of-fit is statistically proved.

Moreover, having an adequate channel model is necessary but not sufficient. Other components of the real-time situational awareness system should also be modeled. There are currently several different designs for real-time situational awareness component. The original design of [24, 25] relies on periodic sampling and communication of vehicle state; in [26] an error-dependent policy is used to reduce the load of real-time situational awareness on the underlying DSRC network. In [27] an error-dependent policy is mixed with a transmission power adaptation scheme to ensure the scalability issue in VANET is avoided as much as possible. Another notable solution [28] uses an adaptation of the sampling and communication rate to avoid congestion in the network. A study of the overall real-time situational awareness system reveals mutual coupling of the communication and estimation processes [29, 30], which prevents a straightforward solution for the scalability issue. Therefore, modeling the mutual effects of the two components of real-time situational awareness component is a critical step in designing a high-performance real-time situational awareness system [29, 30].

Our previous works in [29-31] have shown that the traditional stochastic models, though useful, do not provide enough insight for optimal designs. Mainly due to the fact that performance measures are presented in average forms in the above models, hiding detailed interaction between components. In this study, we offer a new approach to modeling real-time situational awareness components, based on hybrid systems concepts. We use hybrid systems to allow for a unified framework that includes all components without the excessive abstraction of important details. Our objective in presenting this modeling approach is to allow the study of autonomous crashavoidance systems or cooperative vehicle systems (CVS) without ignoring the communication and estimation processes. The new modeling approach provides the capability to jointly and directly examine the effects of the communication and estimation processes in one model, for any given vehicle trajectory and network scenario. This allows for a significantly better understanding of the system behavior when compared to the method of separately analyzing components and then combining the averaged results [29].

Instead of the stochastic Markov-chain model of [31], or the empirical models in [29, 32], we propose to model the system using techniques drawn from the hybrid automata theory. In [33], we presented a preliminary model based on probabilistic timed-automata for the networking/communication component of real-time situational awareness. The main reason for approaching probabilistic timed-automata is that the communication component can be considered as a discrete-event system (DES) [34] because the protocol behind DSRC (i.e., CSMA/CA) operates with integer quantities such as countdown timers. Moreover, many events that control the behavior of the system operate based on instantaneous events such as sending information to other vehicles and sensing the Channel-busy status. The CSMA/CA protocol functions based on some state transitions (e.g., between sending data, receiving data or sensing and waiting for an idle channel. The randomness in CSMA/CA is due to the random back-off procedure as well as random packet arrival patterns and channel sensing results. We employ timed-automata [34-36] due to its features and properties in modeling distributed computation, the presence of timing constraints, and intrinsic randomness in CSMA/CA. In [36], the effect of different computer clocks on the behavior of a distributed application and weakness of timed-automata in modeling these issues are investigated. 
Modeling networking/communication protocols using methods outside queuing theory has been attempted by several researchers in recent years [37-40]. In [37], a probabilistic timed-automata model for the IEEE 802.11 protocol is presented and studied for a two-station case. In this work, two senders contend to broadcast their information, and the authors use PRISM model checker to build and verify the model for different measures. In [38], authors employ stochastic Petri Net to model, analyze the performance, and study all aspects of the IEEE 802.11 medium access control scheme. In [39], the author models the IEEE 802.15.4 CSMA/CA protocol (ZigBee) using probabilistic timed-automata due to the behavior of this type of model in the presence of dense time, non-determinism, and probabilistic choice. PRISM is employed to simulate the model and to check its effectiveness. While these models are useful and have inspired our current work, they mainly consider the communication protocol. In the modeling of real-time situational awareness component, the communication protocol is tightly coupled with and interacts with the estimation process. Moreover, the CVS uses IEEE 802.11 in broadcast mode and in the presence of hidden nodes. In this mode, senders have no feedback to know whether their transmission has been successful or a collision has occurred at some destinations. In our work, we present a model that considers all the above issues using probabilistic timed-automata and hybrid automata.

\subsection{Multi-hop Situational Awareness through Network-aware Double-layer Distance-dependent Broadcast}

V2V communication is a promising technology to be used for creating single-hop and multi-hop situational awareness. Single-hop communication is mostly used in safety applications with strict latency requirements and provides real-time situational awareness; for example, collisionavoidance systems need real-time awareness of their surroundings. Multi-hop communication is more useful for applications that need to disseminate vehicle or road information to distances much farther than a single-hop transmission range and provides and extended range for situational awareness. Challenges and issues of multi-hop broadcast in VANETs are completely different from mobile ad-hoc networks (MANETs) for several reasons [41]. The most important ones are unidimensional movement patterns of the vehicle and rapid changes of topology due to high velocity. Therefore, we only concentrate on existing literature for VANETs.

Different categories of VANETs are introduced in the literature. Understanding these categories facilitates a comprehensive exploration of different issues that single-hop or multi-hop broadcast can address. As mentioned previously, VANETs are categorized into three major groups based on their applications: safety, efficiency, and comfort [42] and the focus of our work, is a multi-hop broadcast protocol for both safety and efficiency applications. VANETs can also be categorized according to their communication patterns: unicast routing, advanced information dissemination, geo-broadcast, information aggregation, and beaconing [43]. Advanced information dissemination uses single-hop broadcast. Geo-broadcast and information aggregation need multi-hop broadcast while they are suitable for efficiency and comfort applications. As considered in this work, beaconing which was originally proposed for single-hop broadcast in safety applications is also used for multi-hop broadcast purposes when it is combined with piggybacking of safety and efficiency data [43-45].

As mentioned in existing surveys on broadcasting in VANETs [46-48], all multi-hop broadcast algorithms have two general steps in common; broadcasting a message to all neighboring vehicles 
and choosing the next forwarder to disseminate the message over multiple hops. Concurrently, these algorithms should try to keep reachability high, while staleness of data and channel load are maintained to be low. Therefore, these two steps are used to categorize the existing works.

The first step, broadcasting of messages to neighbors, is mainly affected by network topology [43, 46]. There are three methods to initiate the broadcast, beaconless immediate transmission, request for relay first, or beaconing. Methods for the multi-hop broadcast of information with beacons are proposed for all topological categories of VANETs, in which the network topology is categorized to disconnected, sparsely connected, and fully connected [49]. However, each of these categories facing different problems and vehicles should switch between different schemes based on their current topology. In the disconnected and sparsely connected networks, the velocities are high so the time span in which the two vehicles can establish a short-range communication is small. Therefore, existing methods should always rely on store-carry-forward mechanisms [50], and since the channel rarely becomes congested in these networks, reduction of staleness is tied to resolve the reachability and intermittent connectivity problem [51-53]. On the other hand, in fully connected networks, which is the case of this work, the speed of the vehicles is relatively low which results in high vehicle density and congested network condition. In these situations, the main problem in front of staleness reduction is scalability and lightweight communication mechanisms to minimize channel load. Moreover, another topological classification for VANETs is based on their distinguished road layouts: Rural roads, highways, and dense city roads which are also known as urban areas [43]. The vehicular network in our case is a multi-lane highway with various traffic flows.

Selection of the next forwarding vehicle is the second step which divides existing methods into six groups [47], which are all distributed methods.

- Table-based: In these methods, each vehicle maintains a list of all neighbors based on their beacons.

- This list is included in transmitted beacon or transmitted separately, and the neighbor with the fewest common neighbors will be the next forwarder [54, 55]. However, sending the long lists along with the messages or separately proliferates packet size and overwhelms the channel as the density increases.

- Vehicles keep the velocity of their neighbors and categorize them into three groups, same road ahead, same road behind, and different road. Then, the farthest vehicle in each group is chosen to be the next forwarder [56]. Nonetheless, the farthest vehicle may not receive the message due to asymmetric channel conditions or it might already change its route.

- Cluster-based: vehicles create clusters in which a cluster head and two gateway vehicles rebroadcast messages [57]. The farthest vehicle is chosen as the next forwarder. However, due to rapid changing of topology, creation, and maintenance of clusters are not viable.

- Topology-based: The topology-based methods use topological information of the network which was discussed completely in the previous step (store-carry-forward mechanism). The next forwarders are the vehicles that stored and carried the information [49-53]. 
- Location-based: These methods are also known as geo-broadcast, in which information should be relayed to a specific area of the road $[58,59]$. Since the goal of these methods is different from common multi-hop broadcast methods, their next forwarder selection policy is different. The nearest vehicle will be the next forwarder in these methods.

- Distance-based: The distance-based methods are semantically different from the distancedependent forwarding which is used in our work. In distance-based methods, the relative distance is used to choose the next forwarding vehicle, which is the farthest vehicle from the source [41, 60-65]. There are two main drawbacks with existing distance-based methods. Independent beaconing is used in almost all of them, in which the farthest vehicle is advantaged on accessing the channel and scheduling its next transmission with a delay, inversely proportional to its distance from the source. Therefore, the transmission rate increases and intensifies the network congestion. Moreover, network conditions have never been taken into consideration in any of the existing works, while neglecting the consequences of these issues has a negative effect on channel load and packet reception.

- Probability-based: The next forwarding vehicle is selected based on a predefined probability function $[53,66,67]$.

- In [66], the highest probability is given to the vehicle with the highest density. Therefore, it has the highest chance to schedule its adaptive independent beacon sooner than others. Thus, other vehicles will not send the message because they already received the retransmission. However, this method is mostly suitable for sparsely connected topologies, as adaptive beaconing with high transmission rate and high density of vehicles can reinforce the congestion in the network.

- The location-based algorithm described in [67], uses the estimated probability of reception for probability function of the next forwarder. This method also uses adaptive beaconing in which each vehicle schedules its next transmission with a delay, inversely proportional to its reception probability. Since closer vehicles have higher reception chance, they will be selected for the next forwarder, which is only consistent with expectations of location-based methods.

- Weighted p-persistence probabilistic broadcasting is used in [53], in which the forwarding probability is calculated as $p_{i j}=D_{i j} / R$, where $D_{i j}$ is the Euclidian distance between vehicle $i$ and $j$, and $R$ is the single-hop communication range. This function does not include density; however, an increase in density will negatively affect the channel load since each vehicle with a distance value close to $R$ will have a high forwarding probability.

In the context of multi-hop broadcast in VANETs, weighted p-persistence probabilistic broadcast, is the only work that combines probability-based and distance-based broadcast methods in order to minimize the staleness. However, it relies on independent beaconing and is not suitable for dense vehicle scenarios, as increasing number of vehicles leads to channel congestion. Apart from that, there are only a few works in MANETs with a combination of both distance-based and probability-based schemes, even though their environment and goals are different, they mostly try to address reachability related problems and do not consider congested network scenarios [68, 69]. 
To the best of our knowledge and based on existing literature surveys, the multi-hop broadcast protocol explained in this work is the only method that utilizes a low amount of channel capacity, leveraging piggyback over single-hop beacons along with a message size control scheme. It also keeps the amount of staleness low by using a network-aware probabilistic distance-dependent approach.

\subsection{Situational Awareness and Hazard Detection for Vulnerable Road Users}

Despite the noticeable efforts of vehicle manufacturers, Vulnerable Road Users (VRUs) such as pedestrians, people with disabilities, cyclists, motorcyclists, public safety personnel, and road workers still account for a significant portion of accident fatalities [70]. Driver distraction and obstruction invisibility, or VRU unawareness of the severity of imminent danger are among the primary reasons behind these fatalities.

VRU safety considerations started around the 1970s by passive safety mechanisms such as head protecting deformable hoods, outside airbags, and helmets, which have become widespread in different parts of current vehicles [71]. Passive safety methods are primarily intended to reduce the severity of the injuries during or after vehicle to pedestrian crashes, as it is not always possible to avoid accidents. In such incidents, vehicles safety features should safeguard not only their passengers but also VRUs. Therefore, almost all of passive safety improvements are taken place in vehicle side. Furthermore, VRUs are practically less flexible to be provided with passive safety equipment.

Nowadays, active safety mechanisms, which are capable of predicting and avoiding vehicle to VRU crashes, have become an essential part of advanced driving assistance systems (ADAS) in premium vehicles. Active safety systems, consist of sensors to observe the surrounding environment of the vehicle, applications to detect probable hazardous scenarios, and actuators to appropriately respond to them. A vision-based active safety mechanism for pedestrian detection is described in [72], in which a rangefinder is utilized to detect pedestrians. However, standalone and non-cooperative active safety approaches may only reduce a fraction of accidents due to perception limitation and obstruction of sensors. Most of the sensors have the line-of-sight (LOS) detection capability up to 150 meters and angle of view of 30 degrees. Thus, occlusion, due to obstruction or severe weather condition, is their primary constraint. The most important sensorbased non-cooperative VRU safety systems have been categorized and surveyed in [73].

Based on the report published in 2014 by NHTSA regarding methodologies for Pedestrian Crash Avoidance/Mitigation System (PCAM), VRUs who had been obstructed and revealed in less than 2700 milliseconds are challenging and could not be detected timely [74]. Thus, non-cooperative active safety mechanisms are unreliable in scenarios in which VRUs appear in front of vehicle less than 2.7 seconds before impact. Consequently, radio communication technologies are adopted to overcome the occlusion dilemma, since they can cope with non-line-of-sight (NLoS) situations. Furthermore, vehicular communication technologies typically have an effective range of 300 meters, are omnidirectional, and are capable of two-way information sharing by nature. Hence, Communication-based cooperative safety methods provide a more extensive situational awareness in comparison with non-cooperative sensor-based methods. 
Different strategies have been proposed for cooperative VRU safety applications based on wireless communication technologies. The most important advantage of cooperative methods is the capability of vehicle and VRU to periodically update their counterpart about their accurate location information. Thus, the performance of cooperative safety application highly depends on the accuracy of global navigation satellite systems (GNSS) such as global positioning system (GPS), Glonass, or Galileo. A project was performed to evaluate the effect of GPS accuracy on VRUs safety systems. Smartphones are utilized as GPS sensors that provide VRUs' positioning data [75]. According to the results, although adequate longitudinal accuracy was attained, reliable lane-level localization of VRUs could not be obtained due to high deviations of lateral information.

Tag-based methods which use radio frequency identification (RFID) tags are described in [76, 77]. In these methods VRUs should carry RFID tags and vehicles should mount a transceiver device to detect the presence and predict the movements of the VRUs. However, the effective communication range of RFID tags is limited to 60 meters [76, 77]. Moreover, in spite of the fact that additional equipment required to be carried and advanced filtering techniques needed to detect the VRUs, tags are not capable of providing much information about the pedestrian status.

Undoubtedly, smartphones are the most prevalent portable communication devices which have become an inseparable part of people's everyday life. Furthermore, compared to any other communication equipment, smartphones have comprehensive human-machine interfaces (HMIs), powerful processors, and extensive multi-media functionalities. Therefore, cooperative VRU safety based on smartphones is an area of increasing interest. Cellular (UMTS/LTE), Wi-Fi Direct, and DSRC technologies have been the primary candidates to provide the connectivity between vehicles and VRUs in different prototypes.

Cellular technology was used to exchange GPS data amongst smartphone, navigation system of the vehicle, and a central server which is responsible for risk assessment and hazard notification based on provided information [78, 79]. A similar approach has been adopted in [80], in which it was assumed that vehicles and pedestrians had sufficiently precise position information. The same authors extended their work in [81] by comparing different combinations of cellular and ad hoc networks to improve the exchange of information between vehicles, smartphones, and central servers. They also proposed some enhancements to filter unthreatened pedestrians without any justifications. Pedestrians who are not moving toward the road are suggested to be filtered. Other information such as movement history or activity context (e.g., talking, texting, or surfing) is also recommended for more detailed filters. Furthermore, it has been demonstrated that the communication delay of cellular networks is in the order of seconds which is unbearable for cooperative pedestrian safety application.

Wi-Fi Direct is a generation of IEEE 802.11 standard which works at $2.4 / 5 \mathrm{GHz}$ frequency. It enables ad hoc communication of Wi-Fi devices without the necessity of an access point (AP). Wi-Fi Direct has a significant reduction of connection establishment delay, from approximately eight seconds to one second, in comparison with conventional AP-based Wi-Fi. In Wi-Fi Direct networks, group owners play the critical role of APs to announce the existence of the network and connect devices to each other. Consequently, as the network formation and group owner negotiation should be renewed if the group owner leaves the network, frequent network reformation might result in an unacceptable delay. Take that into consideration, vehicles and VRUs are not acceptable candidates for the group ownership due to their nonstop and fast dynamics. 
Nevertheless, this technology is not refused by the research community as Wi-Fi Direct capable smartphones have been already commercialized. Moreover, Wi-Fi Direct enabled infrastructures could be placed in intersections to resolve the group owner issue, even though less than ten percent of VRU crashes and one percent of fatalities happen in intersections [82]. General Motors has adopted Wi-Fi Direct to prototype a cooperative pedestrian safety system [83]. The same approach to use Wi-Fi Direct is adopted by authors of [84]. However, their assumption of DSRC and WiFi Direct similarity in not valid. Although, they are both part of IEEE 802.11 standard, they are significantly different in many aspects such as targeted mobility and deployment location [85].

DSRC is a wireless technology specifically designed to support inter-vehicle communications. The main series of standards to support DSRC-based Wireless Access in Vehicular Environment (WAVE) are as follows. The PHY and MAC layers use IEEE 802.11p standard. The IEEE 1609 family of standards are utilized for the middle of the DSRC stack. Finally, the SAE J2735 standard, which is a dictionary that defines a set of application layer message formats, is placed at the top of the stack. The most important of these message formats is the basic safety message (BSM), which is used in V2V safety applications and contains critical vehicle state information [1]. In the latest version of SAE J2735, the Personal Safety Message (PSM) is defined to specifically convey safety information for different types of VRUs. Furthermore, Optional data elements such as path history can also be included in PSMs if needed [86].

DSRC is particularly designed for vehicle safety purposes to have low latency and high interoperability. USDOT is going to mandate the use of DSRC-enabled units in all vehicles, as safety applications based on V2V communications are greatly capable of preventing traffic crashes [87]. Consequently, DSRC is considered as the most legitimate basis for cooperative VRU safety by standard legislators and technical committees [86]. The main barrier of DSRC-based VRU safety services was equipping the VRU smartphones with DSRC. Recently Qualcomm addressed this concern by announcing their capability to override and upgrade existing Wi-Fi firmware to operate in DSRC band without any additional hardware cost. Cooperative vehicle-to-pedestrian (V2P) safety systems based on the Qualcomm DSRC-enabled smartphones have been prototyped by Honda and Hyundai-Kia [88, 89]. The effect of pedestrian side DSRC devices on the channel condition for an intersection is studied by ns-2 simulator [90]. Pedestrian DSRC devices are simulated based on the assumption of being identical to vehicle side devices in all aspect except pedestrian speed, which is not a valid assumption for real world scenarios as smartphone limitations introduce additional constraints to DSRC devices.

\subsection{Situational Awareness Enhancement with Learning-based Driver Behavior Modeling}

The driver is the most important, influential, and informative entity in Intelligent Transportation Systems (ITS). Therefore, Advanced Driver Assistance Systems (ADAS) play a significant role in the realization of Intelligent Transportation Systems (ITS). A revolutionary age of modern driving in the sense of safety and comfort has been initiated by the advent of ADAS equipped vehicles. The first generation of ADAS was designed by virtue of local sensors such as radars and cameras. Local sensors provide a mediocre level of safety due to their limited sensing range and data processing complexity. Moreover, they have significantly underperformed in the presence of occluding obstacles. Vehicle-to-Vehicle (V2V) and Vehicle to Infrastructure (V2I) 
communications have removed this barrier with their omnidirectional and Non-Line-Of-Sight (NLOS) connectivity properties. Consequently, quality of ADAS is improved substantially by V2x communication which has been attained using Dedicated Short Range Communication (DSRC) as a promising technology.

Among ADAS applications, Adaptive Cruise Control (ACC) is one of the most demanding, which improves retention of the driver. In comparison with ACC, Cooperative Adaptive Cruise Control (CACC), which leverages the V2x communication, is more powerful to simultaneously preclude collision and maximize traffic throughput. CACC application should be completely robust against all vehicle maneuvers such as cut-in and cut-out into and from the CACC platoons. Considering that detection and appropriate reaction to unexpected vehicle maneuvers are among the most challenging tasks even in the normal driving situations without CACC imposed constraints. Therefore, while theoretical and technical aspects of CACC have been investigated by researchers, reaction to unexpected vehicle maneuvers, such as cutting into a stable CACC platoon, is still needed for a complete CACC design.

Among unexpected vehicle maneuvers, a unsignaled lane change is one of the most dangerous scenarios that significantly affects safety and platooning performance of CACC application. We specifically concentrate on cut-in maneuvers due to their imminent threat, as a vehicle in a stable CACC platoon has to hard brake when another vehicle performs a lane change into the platoon just in front of it. This hard brake maneuver can lead to a crash or platoon deformation. However, anticipating the cut-in maneuver of neighboring vehicles and predicting the near future behavior can avoid such hard brakes and their consequences. Moreover, a comprehensive CACC should be able to keep the platoon formation against entering or leaving vehicles. Therefore, the performance of CACC in these critical driving scenarios is extremely reliant on the accuracy of driver behavior modeling and lane change prediction becomes an inseparable part of the platooning system design.

Between lane change scenarios, cut-out maneuvers are generally safe for CACC and platooning which can be compensated without any risk. The vehicle behind the leaving vehicle only needs to catch up with its new leader to fill the cut-out gap. The same behavior will then propagate through the rest of the platoon. In contrast, cut-in maneuvers require a more precise tracking and observation as they not only cause platoon deformation but also increase the risk of accidents. Moreover, in safety threatening situations, such as hard braking, safety actuators like automatic emergency braking (AEB) may interrupt other assistance applications like CACC. Thus, it is welldesired to predict cut-in intention of the driver beforehand to prevent threatening situations. Therefore, in our study, we design a learning-based driver behavior model in terms of lane change prediction.

Two main approaches have been employed toward driver behavior modeling in the literature, namely probabilistic methods and classification-based techniques. The main idea behind the first approach is the development of a probabilistic causal framework which tries to relate different essential driving maneuvers in an uncertain way. Dynamic Bayesian Networks (DBNs) and Hidden Markov Models (HMMs) are two prominent probabilistic modeling approaches. Alternatively, classification methods are used to differentiate between distinguishable driver behaviors. For instance, Support Vector Machine (SVM) is a powerful tool which provides a valid discrimination of conceptually different driving movements. 
On the other hand, model generation methods can be categorized based on their accessible sources of information. Cameras and on board detection devices such as radars and LiDARs are assumed as the only information providers in a considerable number of proposed methods in the literature. However, the more recent works are mainly focused on the Vehicle-to-Vehicle (V2V) communication, which is obtainable using DSRC devices, as a supplementary source of information. This technology enables a more precise decision making with imperceptible additional cost.

Authors in [91] developed a hierarchical classifier for observed scenes of the host vehicle. These scenes were then assigned to nodes of the hierarchy in the model to specific a pattern from the top nodes to the leaves. Prediction of surrounding driver maneuvers at a highway entrance, in which a lane change is mandatory within 3 seconds, was the main target of their classifier.

SVM-based methods are proposed to classify lateral actions of drivers based on detection of preparatory behaviors, vehicle dynamics, and the environmental data prior to and during the maneuvers such as lane change [92]. The maneuver is divided into three different stages including inferred environmental intent which is the reason of the maneuver, a lateral intent which is the driver preparation, and lateral action. A separate classification is used for each stage.

An Object-Oriented Bayesian Network (OOBN) is utilized to recognize typical highway driving maneuvers [93]. This approach models different driving maneuvers as vehicle-lane and vehiclevehicle relations on four hierarchical levels which can tolerate uncertainties in both the model and the measurements. The lane marking crossing likelihood and a potential lane change maneuver are represented with the vehicle-lane-marking relations and vehicle-lane relations, respectively. Finally, all possible maneuvers for a pair of vehicles are determined using vehicle-vehicle relations. The effectiveness of this framework is evaluated in lane change maneuvers.

A finite set of driving behaviors are classified and future trajectories of the vehicle are predicted based on currently understood situational context using a filter that was designed utilizing a DBN based model [94]. The situational context was translated to the awareness of vehicle interactions with other traffic participants.

Hidden Markov model (HMM) technique has been widely utilized to associate the observable time series of the vehicle to the unobserved driver intentions sequence during his maneuvers. Some pioneer works in driver behavior modeling, [95, 96], proposed a decomposition of driver behaviors into small scale and large scale categories. The time sequence of unobserved large scale driver actions are assumed to have Markovian property and HMM is suggested as an acceptable method to model this sequence. This modeling approach accuracy was validated by its results of the lane change maneuver prediction. Sensory collected information was used as the observation set in the designed HMM predictor. Moreover, [95] proposed a Kalman filter estimation approach for the small-scale changes of behaviors during each large-scale state.

In another work, a system built on HMM is trained to recognize the driver intention prior to a maneuver using the inter-vehicle and environment perception [97]. The HMM acts as a classifier which discriminates between different maneuvers based on their likelihoods. The proposed method is evaluated for the lane change detection, where the model is trained using the steering angle and yaw rate data. 
Experimental data gathered from cameras and inter-vehicle signals are used for training of an HMM and an asymmetric Coupled HMM (CHHM) to model seven different driver actions [98]. The results found the performance of CHMM, which also can model the interactions of processes, more promising where the HMM could not represent the driver-environment interactions.

Utilizing an HMM, which jointly models a sequence of driver gaze and vehicle dynamics lane changes are detected and categorized into safe or risky maneuvers [99]. The HMM is trained based on the subjective level of risks for a couple of scenarios. The subjective scores and the highest correlation between cumulative HMM log-likelihood ratios, which is defined as the ratio of the safe state likelihood to the risky state likelihood, are calculated as the performance criteria.

An extended version of SVM, namely Relevance Vector Machine (RVM), was employed in [100] to distinguish between lane change and lane keeping maneuvers. A bulky vector of features was constructed by sensory data fused from ACC, side warning assist radars, lane departure warning camera, and head-tracking cameras to characterize driver behavior.

Seven discrete HMMs for seven predefined distinct driving maneuvers was designed to model high level driving actions based on measured values of GPS, velocity, and acceleration [101]. Discretization was performed by quantizing these continuous signals. The number of states for each HMM was separately optimized in the training phase. Test sequence was passed through all seven HMMs and the highest probability was used to select the best maneuver among all candidates.

Using the data from $\mathrm{V} 2 \mathrm{~V}$ communication, two HMMs were utilized to discriminate different types of driver lane change intent, namely dangerous and normal [102,103]. A trajectory prediction stage and an MPC controller were mounted on top of the lane change prediction algorithm to manage reformation of a new CACC string after cut-in.

Feed-forward artificial neural networks are used to predict the trajectory of the vehicle based on its past [104]. The goal was to study the possibility of accurate movement prediction of a lane changing vehicle with an autonomous driving vehicle. They found that using feed forward neural networks and local sensors, the neural network is unable to predict the short-term or long-term trajectory of the vehicle with sufficient accuracy.

A controller for a CACC string which takes into account both $\mathrm{V} 2 \mathrm{~V}$ and non- $\mathrm{V} 2 \mathrm{~V}$ equipped vehicles was designed in [105]. This controller tries to handle cut-in and cut-out scenarios with a smooth reaction to the new condition of the host vehicle lane. The cruise system of the host vehicle works in the ACC mode if its new preceding vehicle is not equipped with a DSRC equipment. Otherwise, the CACC mode is selected to regulate the relative distance and speed. No prediction is performed to detect the cut-in or cut-out scenarios in advance.

\subsection{Contributions}

The key contribution of this dissertation is design and modeling of approaches to enhance situational awareness and create effective sharing of information among vehicles.

In summary, the contributions of this thesis can be listed as: 
1. Situational awareness beyond the line-of-sight with a new modeling scheme for the vehicular communication channel, and a new approach for modeling estimation and communication processes of CAVs in a single framework.

2. Multi-hop situational awareness through a new broadcast protocol for fast broadcasting of traffic information.

3. Situational awareness for vulnerable road users with an end-to-end Vehicle-to-Pedestrian framework.

4. Learning-based driver behavior modeling for situational awareness enhancement.

The above contributions are discussed in the following sections as described below.

Chapter 2 investigate how vehicular communication can provide real-time situational awareness for CAVs beyond line-of-sight. Since modeling of the wireless channel, PHY, and MAC is essential for design and performance evaluation of numerous protocols in vehicular networks, in the first section, we study and provide results for large-scale and small-scale modeling of communication channels in dense vehicular networks. We propose an approach to remove the effect of fading on the deterministic part of the large-scale model and verify its accuracy using a single transmitter-receiver scenario. Two-ray model is then utilized for path-loss characterization and its parameters are derived from the empirical data based on a new proposed method. Afterward, we use $\alpha-\mu$ distribution to model the fading behavior of vehicular networks for the first time and validate its precision by Kolmogorov-Smirnov test for goodness-of-fit. A large Received Signal Strength Indicator (RSSI) data set from a measurement campaign is used to evaluate our claims. Moreover, the whole model is implemented in ns-3 to show the effectiveness of the proposed model in the presence of upper layer network protocols.

Subsequently, a new approach is introduced for modeling the estimation and networking processes of CAVs in a single framework. CAVs relies on real-time situational awareness, which is acquired through cooperation with neighboring vehicles in a wireless network, to predict and respond to hazards. The subsystem that provides situational awareness consists of an estimation process and a communication process. The estimation process is the source of vehicle data that is broadcast through the underlying vehicular network. The performance of vehicle tracking is significantly affected by the performance of the communication network because any issues in sending or receiving information could result in erroneous position estimates and, thus, crashes. It is, therefore, essential to produce models that allow a clear view into the dynamics of these two components. We employ probabilistic timed automata to model the networking component; hybrid automata is then used to combine and model both networking and estimation components in a single framework. We extend the presented hybrid automata to model the broadcast network of NCAS in the presence of heavy hidden node interference. We verify our model's accuracy by comparing it to proven ns-3 simulation models. Model checking for the purpose of studying some features of NCAS is also presented [106].

In Chapter 3, we propose a new broadcast protocol for fast broadcasting of aggregated traffic information over multiple hops. Note that dissemination of traffic information over multiple hops in vehicular networks requires scalability measures that prevent network congestion and avoid 
wasting the network capacity with unnecessary information forwarding. This issue is even more important if such traffic information coexists with critical safety information. In particular, a viable solution for traffic information dissemination is piggybacking compressed information on periodic BSMs. Leveraging this technique, we propose a network-aware, double-layer, distance-dependent protocol for fast broadcasting of aggregated traffic information over multiple hops. The first distance-dependent layer uses a message size control scheme and a probabilistic approach to ensure that forwarding within each hop is as fast as possible. The second layer progressively decreases the forwarding rate of subsequent hops as the distance from the source increases. Jointly, the two layers considerably reduce the overall latency of information while also improving the scalability and robustness of the system. We evaluate the performance of the algorithm and compare it to the existing methods, using ns-3 simulations. The results confirm that significant improvement in performance is possible without complicating the forwarding algorithm [107].

In Chapter 4, based on the most common and injury-prone pedestrian crash scenarios, we extend our work by proposing an end-to-end Vehicle-to-Pedestrian (V2P) framework to provide situational awareness and hazard detection for vulnerable road users. While the development of Vehicle-to-Vehicle (V2V) safety applications based on DSRC has been extensively undergoing standardization for more than a decade, such applications are acutely missing for Vulnerable Road Users (VRUs). Nonexistence of collaborative systems between VRUs and vehicles was the main reason for this lack of attention. Recent developments in Wi-Fi Direct and DSRC-enabled smartphones are changing this perspective. Leveraging the existing V2V platforms, we propose a new framework using a DSRC-enabled smartphone to extend safety benefits to VRUs. The interoperability of applications between vehicles and portable DSRC-enabled devices is achieved through the SAE J2735 Personal Safety Message (PSM). However, considering the fact that VRU movement dynamics, response times, and crash scenarios are fundamentally different from vehicles, a specific framework should be designed for VRU safety applications to study their performance. In this article, we first propose an end-to-end Vehicle-to-Pedestrian (V2P) framework to provide situational awareness and hazard detection based on the most common and injury-prone crash scenarios. The details of our VRU safety module, including target classification and collision detection algorithms, are explained next. Furthermore, we propose and evaluate a mitigating solution for congestion and power consumption issues in such systems. Finally, the whole system is implemented and analyzed for realistic crash scenarios.

In Chapter 5, since V2V communication has a great potential to improve reaction accuracy of driver assistance systems under severe traffic conditions and driver behavior is the most influential source of information for transportation systems. Cooperative Adaptive Cruise Control (CACC) is one of the most powerful mechanisms to preclude collision and maximize traffic throughput in parallel. CACC systems must be designed to be completely robust against all special maneuvers such as cut-in and cut-out into and from vehicle platoons. These special maneuvers behave as system disturbances in the system level framework. To this end, we design a learning-based driver behavior model in terms of cut-in intention detection for CACC systems and integrate it into the estimation component of our situational awareness system. The result of this stage is then passed to a model predictive controller to fine-tune inter-vehicle distances of affected vehicles in the CACC platoon.

Finally, Chapter 6 concludes the dissertation and discusses the remaining work and the possible future directions for this research. 


\section{Chapter 2 Situational Awareness beyond Line-Of-Sight through Vehicular Communication}

Many vehicles have been already equipped with cameras, radars, LIDARs, and ultrasound sensors to detect obstacles or vehicles around them and provide real-time situational awareness. The range of these sensors is tens of meters and is limited to the nearest obstruction. In others words, they can only detect obstacles in the vehicles line-of-sight (LOS). However, in order to resolve and mitigate serious traffic accidents and fatalities, autonomous vehicles and crash avoidance systems need a real-time map of their surrounding beyond their line-of-sight, as many accidents are happening because the driver is blinded and his view is occluded by obstacles. Therefore, wireless communication is needed to add a new layer of certainty and provides situational awareness beyond line-of-sight. Cooperative crash avoidance is an example of a system heavily relying on the real-time situational awareness that is gathered from nearby vehicles over a VANET. The objective of this chapter is to provide a modeling approach for vehicular communication channel and situational awareness component which are essential for design and performance evaluation of numerous protocols in vehicular networks. Section 2.1 describes a new modeling scheme for large-scale and small-scale components of the communication channel in dense vehicular networks. An innovative scheme for a joint modeling of the situational awareness and networking components of CAVs in a single framework is explained in Section 2.2.

\subsection{Composite Channel Modeling of VANETs Using Large RSSI Measurement}

Comprehension and characterization of communication medium have a vital role in the architectural design of communication systems and optimization of their parameters. Moreover, the accurate behavior of physical layer and upper layer applications heavily rely on the fidelity of the model of the communication channel. Therefore, modeling of communication channels has received a great deal of attention in the literature. In particular, modeling of wireless channels is more challenging due to their complicated and unfathomable nature. The complexity of wireless channel modeling essentially comes from diversity and ambiguity of influential factors that affect 
signal propagation in the wireless media. Hence, these factors should be rigorously determined with respect to natural characteristics of the wireless environment. For instance, parameters of an indoor environment model are different from an outdoor one as their movement patterns and surroundings are completely different.

Vehicular ad hoc networks (VANETs), which have been vastly targeted by the research community, are among the most emerging outdoor technologies. Channel modeling of VANETs is also challenging as they are exposed to several complex channel imperfections simultaneously, such as path-loss, shadowing, and small-scale fading [108-111]. Thus, an accurate channel model of vehicular networks in various environments such as highways, urban, and suburban should be determined. For instance, a precise channel model can be used for the validation and analysis of physical layer (PHY) [112], or upper layer protocols and applications through a reliable simulation environment [106, 107, 113].

Movement patterns of transmitters and receivers, their almost equal antenna heights (in contrast with cellular networks with a base-station tower and low receiver antennas), and ad hoc nature of vehicular networks make their propagation modeling fundamentally different from cellular networks. Moreover, due to their fast-changing network topology and environment, the existing models of mobile ad hoc networks (MANETs) cannot be utilized for them [41]. Thus, dedicated channel models are needed to describe vehicular networks behavior at a channel level.

In this section, using a large data set of RSSI values collected from a realistic vehicular measurement campaign, we first propose a new approach to derive path-loss parameters for the two-ray propagation model which takes into account the effect of fading mean on average received signal power. Subsequently, we adopt $\alpha-\mu$ distribution to model the small-scale fading of the vehicular environment and statistically prove its goodness-of-fit with the Kolmogorov-Smirnov test (K-S test). To the best of our knowledge, neither modeling of fading with $\alpha-\mu$ distribution nor statistically proving the goodness-of-fit of any fading model have ever been done in dense vehicular environments. Afterward, we take our model evaluation one step further by testing its results under presence of upper layer protocols in ns-3 which is a realistic network simulator.

The rest of this section is organized as follows. Subsection 2.1.1 describes the measurement campaign configuration. A detailed description of our propagation modeling is presented in Subsection 2.1.2. The model evaluation and ns-3 simulation results are presented in Section 2.1.3.

\subsubsection{Measurement Campaign}

We use the data set collected by the Crash Avoidance Metrics Partnership (CAMP) Vehicle Safety Communications 3 (VSC-3) Consortium, in partnership with the United States Department of Transportation (USDOT). This dataset was collected as part of the V2V safety communications scalability activity in a project to study the Interoperability Issues of Vehicle-to-Vehicle Based Safety Systems (V2V-Interoperability project). Although channel modeling was not the main purpose of the project, RSSI values were collected as metadata. The Atheros DSRC radios based on IEEE $802.11 \mathrm{p}$ standard on $5.9 \mathrm{GHz}$ frequency with $10 \mathrm{MHz}$ band were used for the measurements [114]. 
The field environment is a straight, flat 1400 meters 6-lane highway with 400 equidistant-separated radios. Each radio transmits a fixed-length packet every $100 \mathrm{~ms}$. A constant transmission power is predetermined in all transceiver devices. We use the received signal strength indicator (RSSI), which is recorded for a group of transceivers, to model the communication channel. We use two data sets of field scenarios to validate our proposed model.

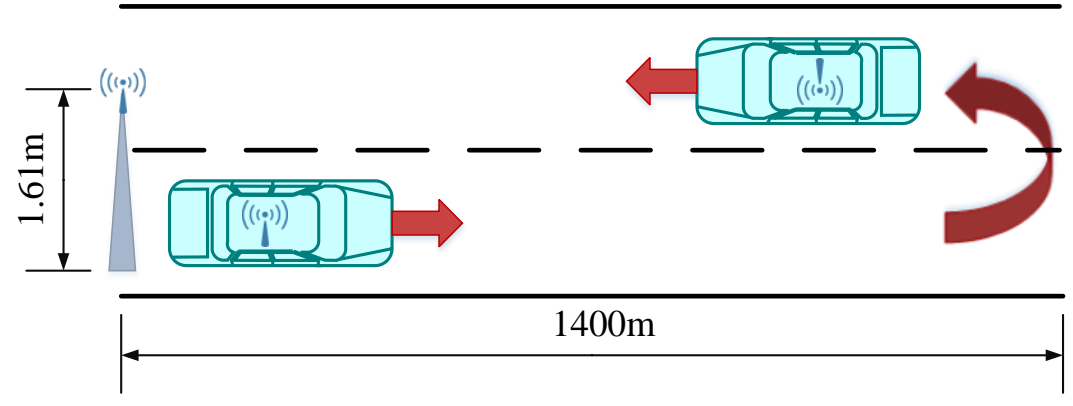

Figure 2-1- Touchstone Scenario

The first scenario is the touchstone scenario by which we derive the environment-related parameters of the two-ray path-loss model and verify our path-loss exponent evaluation method (Figure 2-1). The touchstone is a single transmitter-receiver scenario in which the transmitter is mounted on a vehicle at the height of 1.61 meters. The receiver is mounted on a stand with the same height at the beginning of the road where the vehicle starts. The transmitting vehicle drives away from the stationary receiving vehicle on a straight path with the constant speed of $22 \mathrm{~km} / \mathrm{h}$ and turns around toward it at distance of 1400 meters. The packet transmission rate and the transmission power are set to $10 \mathrm{~Hz}$ and $18 \mathrm{dBm}$, respectively.

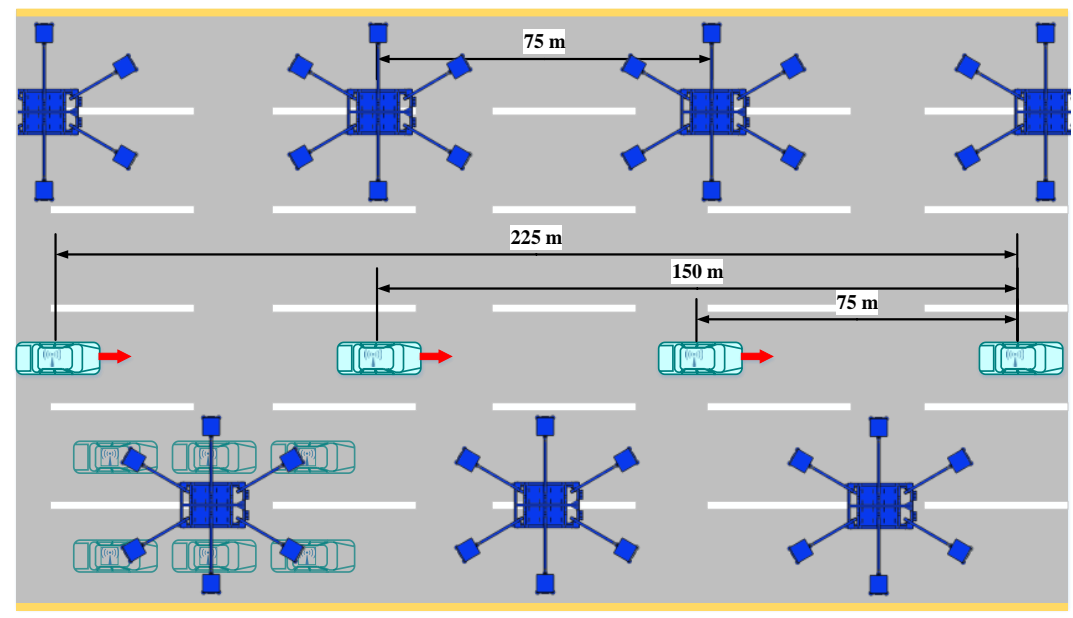

Figure 2-2- 400-car Scenario

The second scenario is the 400-car scenario in which four moving vehicles follow each other and keep 75 meters distance in the middle lane. Each vehicle is equipped with two transceivers on the rooftop at the height of 1.61 meters. The rest of transceivers are placed stationary along the road 
at the same height on top of 65 carts. 6 transceivers are mounted on each cart to play the role of actual vehicles as shown in Figure 2-2. The carts are equidistantly separated with 75-meter distance on four side lanes. The packet transmission rate and power of 400 -car scenario are $10 \mathrm{~Hz}$ and 20 $\mathrm{dBm}$, respectively. Multiple trials of the 400-car scenario with different configurations exist which are used to verify the proposed path-loss and fading models.

\subsubsection{Propagation Modeling}

Communication channel determines how the transmitted signal is changing as it propagates. Although it is impossible to exactly identify the behavior of channel, propagation models have tried to predict the received signal strength to a great extent. As known from the propagation model of a signal in a wireless communication channel, the received power from a transmitter is affected by three well-known factors, path-loss, shadowing, and fading. Log representation of received signal strength at an arbitrary distance $d$ can be written as:

$$
P_{r}(d)=P_{t}-L_{L S}(d)+g_{P, d B}(d B)+X_{\sigma}
$$

where $P_{t}$ is the transmission power in $\mathrm{dB}, L_{L S}(d)$ is the deterministic path-loss at distance $d, g_{P, d B}$ is the small-scale fading, and $X_{\sigma}$ is the zero-mean shadowing with standard deviation of $\sigma$. As carts do not represent the actual obstruction and dimension of 6 vehicles, the environment is sparse and free from any large objects to cause shadowing, except for the four vehicles. Therefore, an accurate shadowing model cannot be derived from our experiments for real highway scenarios.

Considering the setup of touchstone and 400-car scenarios with straight, flat road and vehicles with antennas of the same height, there always exist a dominant direct line-of-sight (LOS) ray and a ground-reflected ray between each transmitter-receiver pair. Therefore, two-ray ground reflection model is the best candidate to model path-loss in vehicular networks $[6,115]$. The following section briefly describes the two-ray model and our proposed approach to finding its parameters.

\subsubsection{Two-ray path-loss model}

Path-loss is a deterministic model that determines the amount of loss in the average received signal strength at a specific distance of the transmitter. As mentioned, two-ray ground reflection is widely used for vehicular networks which can be found as

$$
L_{L S}\left(d ; \gamma, \epsilon_{r}\right)=10 \times \gamma \times \log _{10}\left(4 \pi \frac{d}{\lambda}\left|1+\Gamma_{\perp} e^{i \varphi}\right|^{-1}\right)
$$

where $\alpha$ is the path-loss exponent, which is introduced as an empirical environment-dependent adaptation of non-ideal channel conditions and assumed to be 2 in ideal channel conditions [115, 116]. $\lambda=\frac{c}{f}$ is the signal wavelength corresponding to the transmitted signal with central frequency of $f$ that is propagating in the environment with the speed of $c$. In the above equation, the reflection coefficient $\Gamma_{\perp}$ can be found as

$$
\Gamma_{\perp}=\frac{\sin \theta-\sqrt{\epsilon_{r}-\cos ^{2} \theta}}{\sin \theta+\sqrt{\epsilon_{r}-\cos ^{2} \theta}}
$$


where $\epsilon_{r}$ is a fixed, unit-less constant dependent on the reflection medium. $\sin \theta=\frac{h_{t}+h_{r}}{d_{r e f}}$ and $\cos \theta=\frac{d}{d_{r e f}}, d_{r e f}=\sqrt{d^{2}+\left(h_{t}+h_{r}\right)^{2}}$ are dependent on $h_{t}$ and $h_{r}$ which are the heights of the transmitter and receiver antennas, respectively. Furthermore, the phase difference of the two interfering rays, $\varphi$, can be found as

$$
\varphi=2 \pi \times \frac{d_{l o s}-d_{r e f}}{\lambda}
$$

where $d_{l o s}=\sqrt{d^{2}+\left(h_{t}-h_{r}\right)^{2}}$.

Therefore, the path-loss model defined in Equation (2.2) has two unknown environment-dependent parameters of $\gamma$ and $\epsilon_{r}$ that should be found based on the empirical data.

Nonlinear regression methods are used previously to derive path-loss parameters from empirical data in single transmitter-receiver scenarios [19, 115]. Although, it is possible to use such methods for simple scenarios such as the touchstone, they are not reliable in more complicated scenarios due to the significant effect of small-scale fading mean. Small-scale fading cannot generally be assumed as zero-mean, so the mean affects the empirical average received signal strength. Therefore, we propose the following approach to determine the values of $\gamma$ and $\epsilon_{r}$.

The empirical average RSSI (in $\mathrm{dBm}$ ), denoted as $\left\langle P_{r}(d)\right\rangle$, for each distance $d$ (in meters) is assumed to be equal to expected value of received signal strength

$$
\left\langle P_{r}(d)\right\rangle=E\left[P_{r}(d)\right]=P_{t}-L_{L S}(d)+E\left[g_{P, d B}(d B)\right]
$$

As fading characteristics do not change in close distances, for each distance $d^{\prime}$, which is relatively close to $d$

$$
\left\langle P_{r}\left(d^{\prime}\right)\right\rangle-\left\langle P_{r}(d)\right\rangle=L_{L S}(d)-L_{L S}\left(d^{\prime}\right)=10 \times \gamma \times \log _{10}\left(\frac{d}{d^{\prime}} \times\left|\frac{1+\Gamma_{\perp, d^{\prime}} e^{i \varphi_{d^{\prime}}}}{1+\Gamma_{\perp, d} e^{i \varphi_{d}}}\right|\right)
$$

\subsection{Determining $\epsilon_{r}$}

The value of $\epsilon_{r}$ is determined prior to $\gamma$ as it only depends on the natural properties of reflecting surface. Thus, the touchstone scenario is used to this end as it has less complexity and exactly the same environmental properties. Similar to other single transmitter-receiver scenarios, channel condition is assumed to be ideal and path-loss follows a two-ray model with exponent value of $\gamma=2$, as the environment of this scenario is an open area without any obstacles and a dominant LOS component always exists. Theoretically, using Equation (2.6) for any arbitrary pair of distinct distances $d$ and $d^{\prime}$ should yield to an identical value of $\epsilon_{r}$. However, the obtained value may vary for each pair of distances due to imperfection of empirical data. Therefore, statistical mode of the distribution of obtained $\epsilon_{r}$ values is chosen as the best approximation of the actual value. 


\subsection{Determining $\gamma$}

A similar method is used to determine the value of $\gamma$ which can be derived using Equation (2.6) based on the figured value of $\epsilon_{r}$ from the preceding subsection. The touchstone and 400-car scenarios are conducted in the same field, in which the value of $\epsilon_{r}$ is fixed. Hence, the corresponding $\gamma$ value of each scenario can be determined using previously calculated $\epsilon_{r}$. Similar to $\epsilon_{r}$ calculation approach, the obtained value may vary for any arbitrary pair of distinct distances $d$ and $d^{\prime}$ due to imperfection of empirical data. Therefore, statistical mode of the distribution of obtained values of $\gamma$ is selected as the most accurate approximation of the actual value. The derived values for both scenarios are reported in the results section. Utilizing brute-force regression method in touchstone scenario and achieving the same value of $\gamma$ and $\epsilon_{r}$ approves the validity of our approach. Moreover, our approach is valid in more complicated scenarios such as 400-car, whereas regression method is not reliable as it neglects the effect of fading mean $\left(E\left[g_{P, d B}(d B)\right]\right)$.

\subsubsection{2 $\boldsymbol{\alpha}$ - $\boldsymbol{\mu}$ fading model}

The generalized gamma or Stacy distribution was originally derived in 1962 [117]. The probability density function (PDF) of generalized gamma covers some of the most widely used distributions such as Rayleigh, gamma, one-sided Gaussian, Nakagami-m, exponential, and Weibull as special cases. The $\alpha-\mu$ distribution in wireless communication context is a rewritten form of generalized gamma distribution with change of variables, which was derived by Yacoub [118, 119], based on dedicated characteristics of the wireless medium, such as field non-linearity. The PDF of $\alpha-\mu$ distribution is calculated as

$$
f_{x}(x ; \alpha, \mu, \Omega)=\frac{\alpha \mu^{\mu} x^{\alpha \mu-1}}{\Omega^{\alpha \mu} \Gamma(\mu)} \exp \left(-\frac{\mu x^{\alpha}}{\Omega^{\alpha}}\right), x \geq 0, \alpha>0, \mu>0,
$$

Where

$$
\begin{gathered}
\mu=\frac{E^{2}\left[x^{\alpha}\right]}{E\left[x^{2 \alpha}\right]-E^{2}\left[x^{\alpha}\right]^{\prime}} \\
\Omega=\sqrt[\alpha]{E\left[x^{\alpha}\right]} .
\end{gathered}
$$

The $\alpha-\mu$ distribution is one of the most general distributions which can model both fast fading and shadowing effects concurrently [120].

As aforementioned, Nakagami- $m$ distribution is the most common fading model in the vehicular networks [14, 19, 21-23, 121]. However, the main obstacles and scatterers in vehicular networks, especially in highway scenarios [20], are other vehicles. Thus, homogeneity of scatterers is not a valid assumption as the field scatterers are correlated and not distributed in the field in a complete uniformly random manner. The field non-linearity happens due to spatial correlation of scattering surfaces in contrast with assumption of their uniform distribution. The $\alpha-\mu$ distribution derivation paradigm is mainly proposed to overcome the field non-linearity [118]. While it seems reasonable to leverage the field non-linearity property in vehicular environments, which is also statistically verified in this work, the $\alpha-\mu$ distribution has never been used in VANETs beforehand. 
Parameter estimation of $\alpha-\mu$ distribution has been studied extensively in the literature [122-124]. The two vastly adopted approaches are maximum likelihood and moment generating function estimators $[125,126]$. As the RSSI values in our scenarios are recorded in $\mathrm{dBm}$ and quantized to the nearest integer value, a parameter estimator of log distribution is more desirable to eliminate the numerical truncation error due to log to linear conversion. A set of parameter estimators for $\log \alpha-\mu$ distribution, $f_{l}(l)$ where $l=A \ln x$ and $A=\frac{20}{\ln 10}$, based on $\log$ moments and least square error are proposed in $[120,123]$. We are employing these equations to obtain distribution parameters of fading model for our empirical data. It can be noted that the results of $\log$ representation of moment generating functions are more accurate compared to regular moments [120].

The second and third central moments of the $\log \alpha-\mu$ distribution are used to derive the estimators

$$
\begin{aligned}
& \mathcal{M}_{2}=E\left[(l-\bar{l})^{2}\right]=\left(\frac{A}{\alpha}\right)^{2} \psi^{\prime}(\mu), \\
& \mathcal{M}_{3}=E\left[(l-\bar{l})^{3}\right]=\left(\frac{A}{\alpha}\right)^{3} \psi^{\prime \prime}(\mu),
\end{aligned}
$$

where $\psi(x)=\frac{\partial \ln \Gamma(x)}{\partial x}, \psi^{\prime}(x)$, and $\psi^{\prime \prime}(x)$ are the psi function and its first and second order derivatives, respectively [127]. The $\widehat{\tau}$ estimator is defined as [123, 124]

$$
\widehat{\tau}=\frac{\widehat{\mathcal{M}}_{2}^{\frac{3}{2}}}{\widehat{\mathcal{M}}_{3}} \triangleq \frac{\left(\psi^{\prime}(\mu)\right)^{\frac{3}{2}}}{\psi^{\prime \prime}(\mu)}
$$

where $\widehat{\mathcal{M}}_{2}$ and $\widehat{\mathcal{M}}_{3}$ are empirical second and third central moments of the log $\alpha-\mu$ distribution, respectively. The value of $\mu$ can be estimated as

$$
\hat{\mu}=\left\{\begin{array}{l}
\widehat{\tau}^{2}+\frac{1}{2}, \quad \hat{\tau} \leq-2.85 \\
-0.0773 \widehat{\tau}^{4}-0.6046 \widehat{\tau}^{3}-0.7949 \widehat{\tau}^{2} \\
-2.4675 \widehat{\tau}-0.9208,-2.85<\widehat{\tau} \leq-0.6 \\
-132.8995 \widehat{\tau}^{3}-232.0659 \widehat{\tau}^{2} \\
-137.6303 \widehat{\tau}-27.3616,-0.6<\widehat{\tau}<-0.5
\end{array}\right.
$$

Accordingly, $\alpha$ and $\Omega$, can be estimated as

$$
\begin{gathered}
\hat{\alpha}=A \sqrt{\frac{\psi^{\prime}(\hat{\mu})}{\widehat{\mathcal{M}}_{2}}} \\
\widehat{\Omega}=\sqrt[\widehat{\alpha}]{E\left[\exp \left(\frac{\hat{\alpha} \hat{l}}{A}\right)\right] .}
\end{gathered}
$$




\subsubsection{Model Evaluation}

Calculated parameter values for suggested model based on the empirical data and its statistical evaluation are presented in this section. Moreover, the ns-3 simulation results are shown to evaluate the performance of the model with the presence of upper layer protocols.

It can be noted that the DSRC radio carrier-sensitivity has been tested by the radio vendor, and it turns out that the received packets with RSSI values less than $-94 \mathrm{dBm}$ cannot be correctly detected and are lost in most cases. Therefore, the number of received data points in the empirical observations for higher distances is too low, and the data itself is not representative since it has been pruned by the DSRC receivers to only contain correctly received frames. In other words, we do not have access to a representative set of channel realizations for higher distances as the received packets with RSSI values below the carrier-sensitivity threshold had been truncated from the data points. Hence, to avoid the accuracy of the model being affected, despite the existence of partial data points for the whole field test range, the model derivation is performed for distances up to 800 meters and 400 meters for touchstone and 400 -car scenarios, respectively.

\subsubsection{Touchstone scenario}

As mentioned, the value of $\epsilon_{r}$ is determined from the touchstone scenario. Furthermore, the validity of our proposed approach is verified by comparing its results with regression based methods as a benchmark. Based on the ideal channel condition assumption in single transmitterreceiver scenarios, path-loss follows a two-ray model with exponent value of $\gamma=2$. The statistical mode of the distribution of obtained $\epsilon_{r}$ values for all distance pairs is shown in Figure 2-3 with respect to bin size of 0.01 . Therefore, the value of $\epsilon_{r}=1.01$ is chosen for our measurement campaign environment.

The same method is used to verify the value of $\gamma=2$ based on the figured value of $\epsilon_{r}=1.01$. The statistical mode of the distribution of obtained values of $\gamma$ is shown in Figure 2-4. The value of $\gamma=2$ is consistent with our assumption.

Moreover, brute-force method with least mean square error criteria is used to simultaneously calculate the values of $\gamma$ and $\epsilon_{r}$ in touchstone scenario to verify the validity of our approach. The values of $\epsilon_{r}=1.01$ and $\gamma=2.01$ are computed for regression method. Average RSSI data points of touchstone scenario and two-ray path-loss models with parameters derived from two approaches are illustrated in Figure 2-5. Note that the RSSI values are averaged over one-meter bins. 


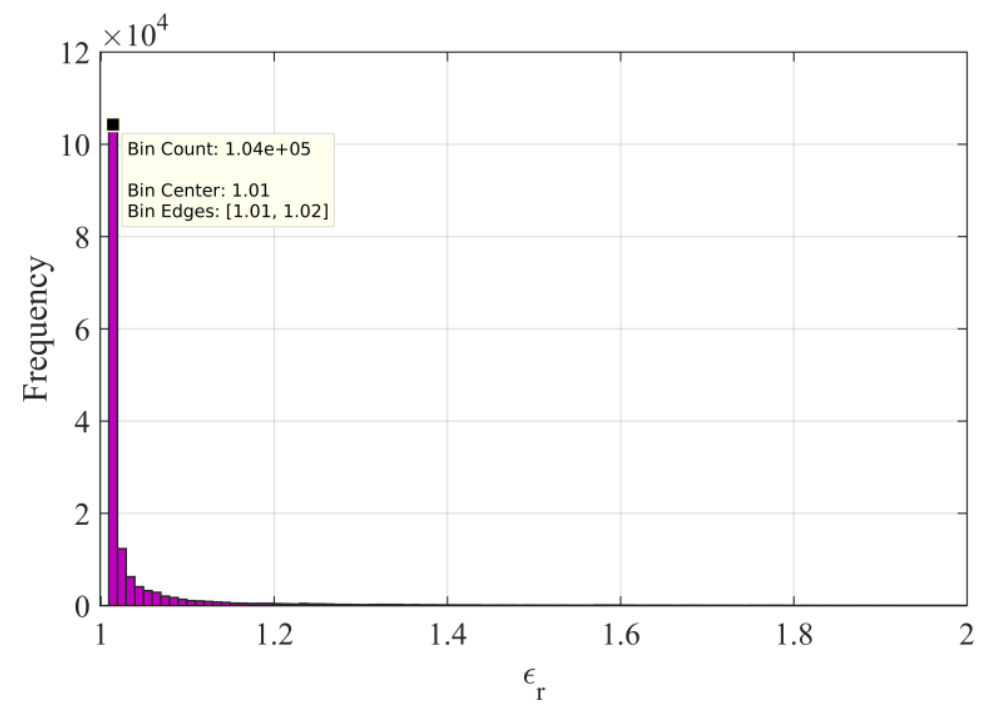

Figure 2-3- Histogram of calculated $\epsilon_{r}$ values from touchstone scenario

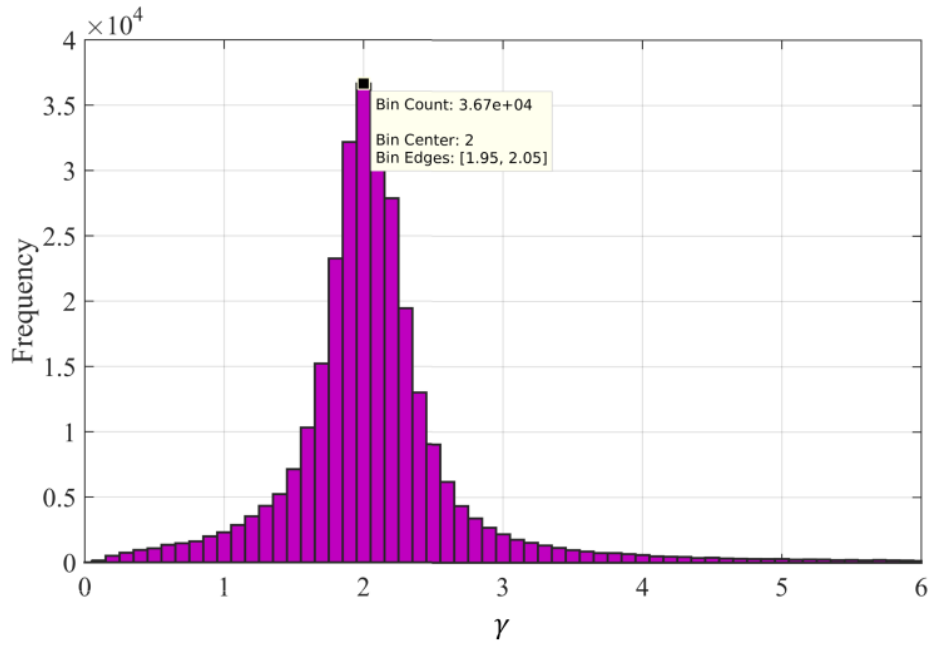

Figure 2-4- Histogram of calculated $\gamma$ values from touchstone scenario 


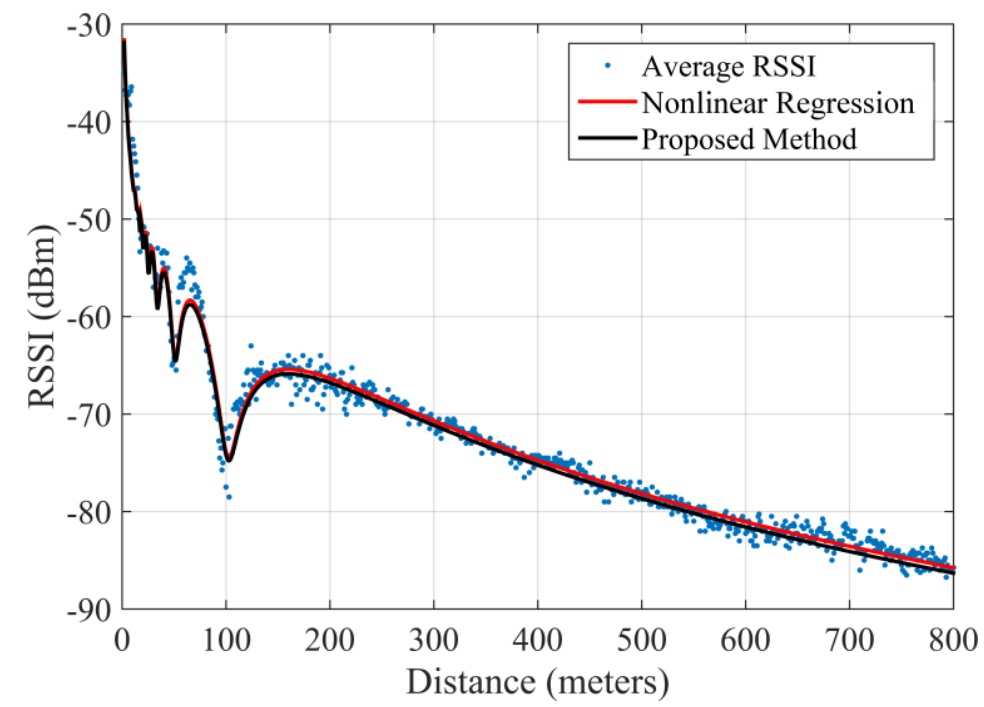

Figure 2-5- Validation of the proposed approach using one-meter bin averaged RSSI values of touchstone scenario

\subsubsection{400-car scenario}

\subsection{Path-loss model evaluation}

Using the aforementioned method, the value of $\gamma$ is calculated for 400-car scenario based on the value of $\epsilon_{r}=1.01$. Figure 2-6 shows the histogram of the distribution of derived values of $\alpha$ along with the most frequent bin, which is the chosen $\gamma=2.1$.

\subsection{Fading model evaluation}

The Path-loss model found in Step 1, with the values of $\epsilon_{r}=1.01$ and $\gamma=2.1$, is subtracted from the original RSSI data points to find the nondeterministic part of data. The aforementioned logmoment estimators are employed to derive the $\alpha$ - $\mu$ fading model parameters. In order to avoid overfitting of the model, the range of 400 meters is divided into 5-meter distance bins, for which a representative set of empirical data is available, and the best set of model parameters are found for each bin. 


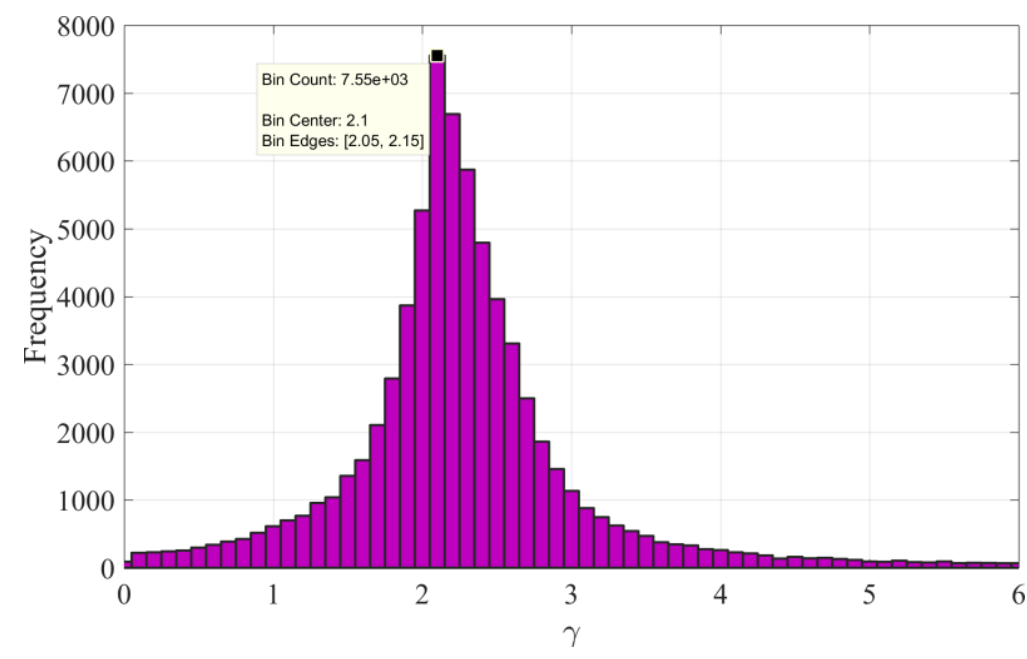

Figure 2-6- Histogram of calculated $\gamma$ values from 400-car scenario

In order to demonstrate the accuracy of the proposed model and evaluate its goodness-of-fit, the same number of data points for each 5-meter distance bin are regenerated using an $\alpha-\mu$ distribution with estimated parameters of that bin.

The RSSI values of each 5-meter bin are represented as a box plot in Figure 2-7. The field box plots are shown in blue and the regenerated points based on the derived model are depicted in red. The central black mark of each box is the median, the 25 th, and 75 th percentiles are the edges of the box, and the whiskers extend to the most extreme non-outlier data points corresponds to approximately 99.3 percent coverage if the data points are assumed to be normally distributed.

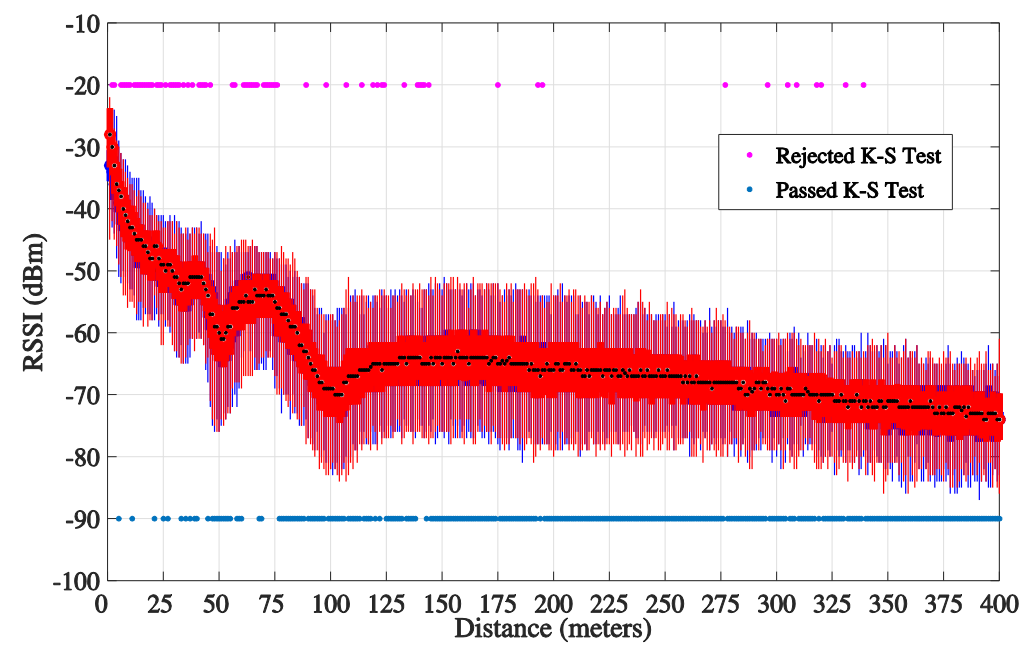

Figure 2-7- Box plot of empirical data (blue) versus regenerated points from $\alpha-\mu$ distribution (red) in 400-car scenario along with K-S test result of each five-meter distance bin. 
Kolmogorov-Smirnov test (K-S test) is a hypothesis testing approach for goodness-of-fit evaluation of a theoretic distribution with an empirical data set [128]. K-S test is widely employed in the literature due to its two important characteristics [19, 120]. First, K-S test statistic is independent of the actual CDF of the empirical data. Second, binning size does not affect the validity of the $\mathrm{K}-\mathrm{S}$ test results. The empirical $\mathrm{CDF}, F_{E}(x)$, is defined as

$$
F_{E}(x)=\frac{1}{n} \sum_{i=1}^{n} I\left(x_{i} \leq x\right)
$$

where $I\left(x_{i} \leq x\right)$ stands for the indicator function which equals to one when the $i^{t h}$ empirical data sample $\left(x_{i}\right)$ is less than or equal to $x$ and zero otherwise, and $n$ is the size of whole empirical data set. In other words, $F_{E}(x)$ works as a normalized counter of empirical data set elements based on the $\mathrm{CDF}$ definition. The main concept behind the K-S test is the similarity judgment of empirical $\mathrm{CDF}$ and hypothesized CDF based on the maximum value of their absolute distance or vertical discrepancy. The maximum vertical discrepancy between these two CDFs is known as the K-S test statistic and denoted as $D$ :

$$
D=\max _{x}\left|F(x)-F_{E}(x)\right|
$$

where $F(x)$ is the hypothesized $\mathrm{CDF}$ for the empirical data.

K-S test results for the 400-car scenario RSSI values versus the $\alpha-\mu$ distribution are also illustrated in Figure 2-7. The empirical data set is divided into five-meter bins and the parameters of $\alpha-\mu$ distribution are derived for each bin, separately. The number of data points for each regenerated bin is equal to its corresponding bin in empirical data to perform a valid K-S test. Bin-wise pass and fail results of performed K-S test with the test significance level of $\alpha=0.01$ are depicted in Figure 2-7 in navy blue and purple, respectively. The statistical assessment strongly supports our assumption about applicability of $\alpha-\mu$ fading model on dense vehicular networks.

We have implemented both large and small-scale propagation models in ns-3, which is a realistic network simulator, to demonstrate the effectiveness of our proposed model.

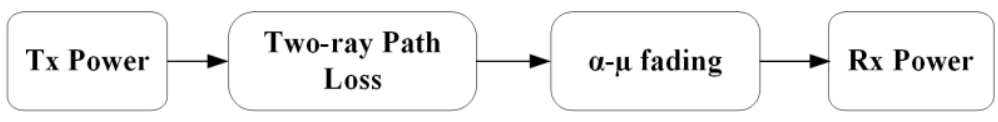

Figure 2-8 shows the transmission-reception procedure in ns-3.

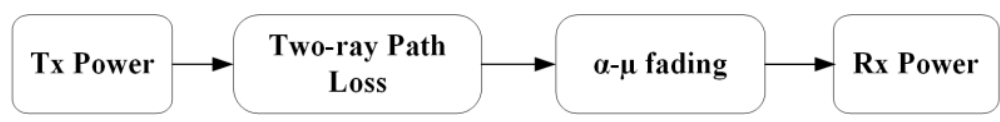

Figure 2-8- Steps of model implementation in ns-3

The box plot comparison of simulation results versus field data is shown in Figure 2-9. 


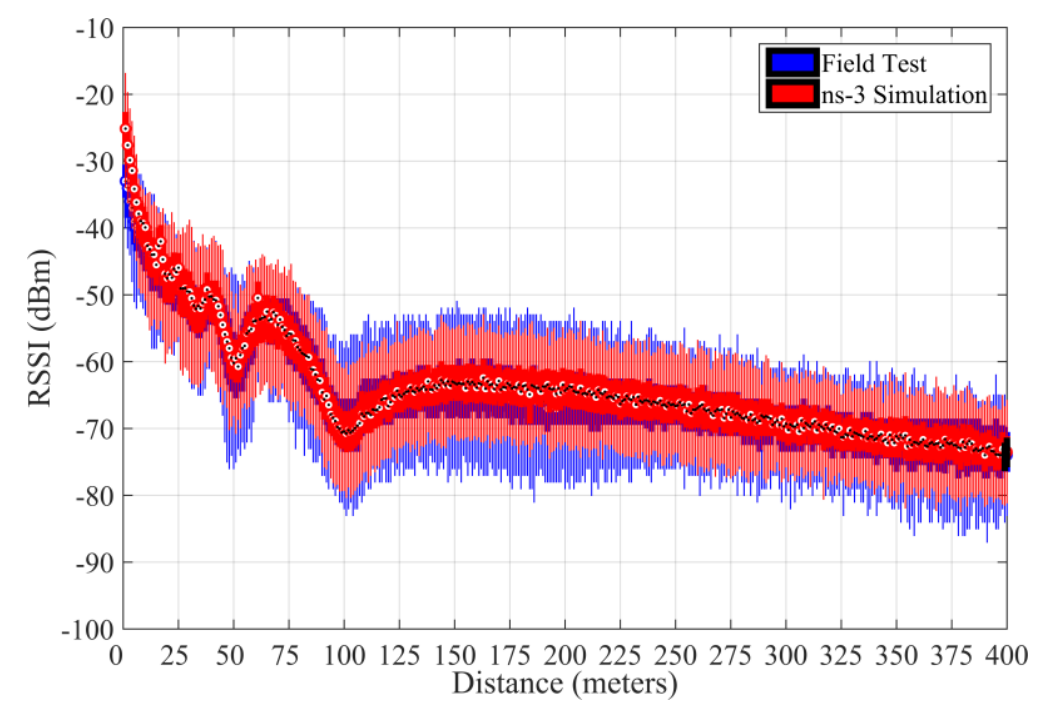

Figure 2-9- Box plot of empirical data (blue) versus ns-3 simulation results (red) in 400-car scenario.

\subsection{Modeling of Real-time Situational Awareness Component of CAVs}

Automated crash avoidance is one of the most important parts of any autonomous driving system. The networked crash avoidance system (NCAS), which is also called cooperative crash avoidance system, is a flavor of automated crash avoidance that relies on cooperation and coordination among vehicles that are within a certain range of each other. Crash avoidance is based on real-time situational awareness information that is gathered from nearby vehicles over a wireless local area network. Autonomous driving or crash avoidance systems comprising of many subsystems. The coordination, integration, and control of these subsystems, as well as the communication among vehicles, and their complex movement dynamics introduce a lot of challenges in the study and modeling of the system. In order to achieve autonomous driving, several functionalities must be integrated, including real-time mapping or situational awareness service, which generates a realtime map of the surrounding of a vehicle, position estimation, and control.

Each real-time situational awareness component is composed of two subcomponents: communication and estimation. Communication is based on dedicated short-range communication (DSRC) technology, one of the enabling technologies of vehicular ad-hoc networks (VANETs) [114]. DSRC uses CSMA/CA (Carrier Sense Multiple Access with collision Avoidance) to share the wireless channel among neighboring vehicles (up to distances of few hundred meters). The CSMA/CA communication protocol specifies how vehicles should coordinate access to the shared wireless channel within the transmission range of each other. There are many issues with using a shared channel in vehicular broadcast networks; the scalability issue is a well-known problem and refers to the fact that in crowded networks the total capacity of the network tends to zero, failing real-time situational awareness altogether [27]. An important reason for such failure is the hidden nodes phenomenon, which happens when two nodes outside the transmission range of each other 
transmit simultaneously to nodes in between them. These VANET issues will adversely affect the estimation process if the network is not properly managed. In this subsection, we present a hybrid model that considers all the above issues using probabilistic timed-automata and hybrid automata.

Our choice of hybrid automata method is also inspired by recent literature on modeling cyberphysical systems [129-131]. It is generally recommended that Hybrid automata (or hybrid systems) modeling seems to be rational because the physical processes could be defined in terms of differential equations, while communication and computation could be represented in terms of timed-automata, state machines, data flows, discrete events, etc. [132].

In our modeling effort, we employ the estimation process that has been introduced and verified in $[26,27,30]$. This method is currently under test by the automotive industry to replace the original design of periodic transmission of state information [25]. The unified modeling of the components of communication and estimation facilitates a more precise and straightforward study of the characteristics and properties of the system and provides an opportunity for separate and mutual performance analysis of each component through model checking. To model the communication component, we consider the DSRC broadcast network in two scenarios; first we consider the case of all vehicles in being transmission range of each other (no hidden node effect) to derive the basic model; we then extend the model to cover the hidden node case, which is the prevalent situation in crash avoidance system (CAS) and CVS networks.

Subsection 2.2.1 provides a brief overview of the system. In Subsection 2.2.2, the model for each component is separately discussed, and a hybrid model is presented to combine both estimation and communication. In Subsection 2.2.3, the hidden node phenomenon and its model are investigated. The validation of the proposed models in comparison with ns-3 results is presented in Subsection 2.2.4.

\subsubsection{System Descriptions}

NCAS and CVS systems are designed based on broadcasting information through a shared channel and providing mechanisms for each vehicle to estimate and track the state (e.g., position and speed) of other vehicles. Vehicles broadcast their state information in a neighborhood around them, as shown in Figure 2-10. For this purpose, each vehicle is equipped with DSRC radio, GPS, and onboard sensors. The real-time situational awareness encompasses all subsystems that are used for tracking of neighboring vehicle positions. The main two components of real-time situational awareness that need to be modeled are the networking/communication process and the estimation process.

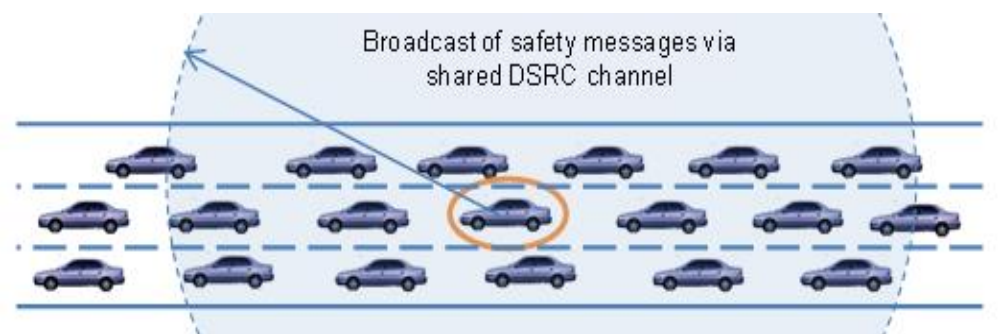

Figure 2-10- Broadcasting the information in CVS systems. 
The networking/communication process is responsible for disseminating state information of vehicles, and the estimation process samples the information in the sender vehicle (message generation) and recovers communicated states at the destination vehicles (state tracking). Figure 2-11 shows these components and their tasks.

It is clear that performances of these two components are related to each other. The performance of the networking process highly depends on the load of information presented to the network by the estimation process of each vehicle (because the performance of CSMA/CA protocol degrades if the amount of load increases). The performance of the estimation process is affected by the dynamical order of its process and also the performance of the communication process because the estimation process recovers the state information based on the successfully communicated state information at the receiver.

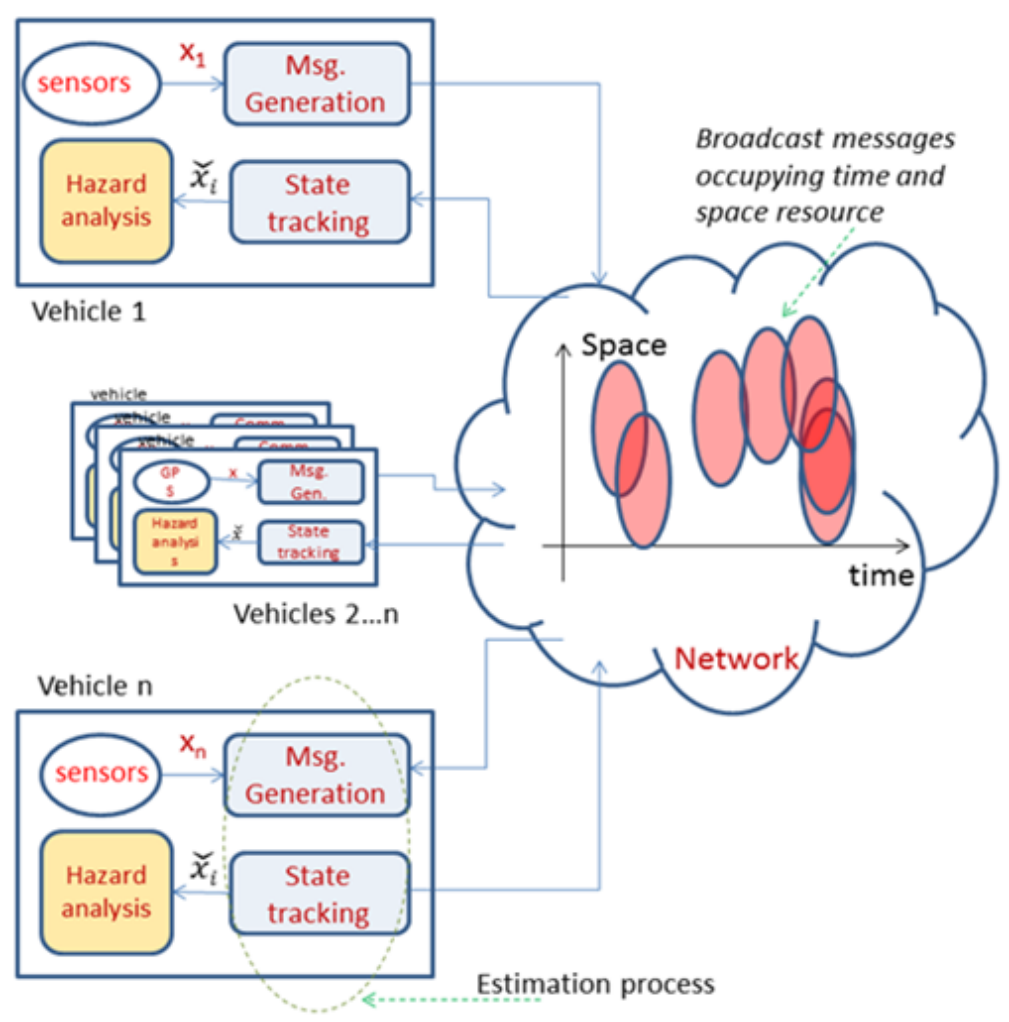

Figure 2-11- Two different components of real-time situational awareness.

Two different scenarios can be considered when modeling the networking component: all vehicles in each other's transmission range, and vehicles interacting only with neighboring vehicles up to a certain distance. When all vehicles are in range, the system follows a rule that situational awareness is required for all vehicles, and each vehicle is assumed to receive state information from all other vehicles (a fully connected network). This is only a hypothetical scenario and is used to create a basic model for the communication component. In the second scenario, the situational awareness is assumed to be required for all neighboring vehicles up to a certain distance; therefore the range of communication and the operation of CSMA/CA will be adjusted 
accordingly. The second scenario is the realistic case of a CAS or CVS and covers the hidden node phenomenon.

The estimation process of real-time situational awareness could use many different policies; in this work we consider the promising error-dependent policy of [26], which is currently under consideration by industry for adoption in the probabilistic form presented in [27].

\subsubsection{Model Description for the Fully Connected Network}

In this section, we consider a fully connected network and develop a probabilistic timed-automata (PTA) model for each sender, receiver, and channel. A hybrid automaton is then presented, that consists of estimation process as a continuous-time process and a networking /communication process model as a discrete-time process for the condition of all vehicles being within range (fully connected network).

\subsubsection{Communication component model}

The networking/communication component of real-time situational awareness in CVS system is based on the IEEE 802.11 standard in broadcast mode. Since the communication process is a discrete procedure, we model the communication process as a sequence of discrete processes and events. Furthermore, the communication process is random; therefore, probabilistic timedautomata is used to model the communication part. The probabilistic timed-automata model is characterized by a six-tuple as follows:

$$
P T A=\left\{S, T, I_{0}, \xi, \Sigma(s), p r o b\right\}
$$

Where,

- $\quad S$ is a finite set of states (location) of the PTA models. The communication model at the sender, receiver, and channel has different states to model the transmission and reception of the information by these states. In each sender, the model starts from wait until free and ends after the model leaves the transmit state. The models simulate the actual communication process, based on the discrete jumps (transitions) between these states.

- $\quad T$ is a set of clocks. Transitions between states occur based on the clocks assigned to them. When the model enters a certain state, its clock is fired and counts from zero to the final clock value.

- $I_{0} \in S$ is the initial state (location). In each sender, the model starts from wait until free and ends after the model leaves the Transmit state in each try of broadcasting the data. The channel model starts from free, and the receiver model starts from the waiting state.

- $\quad \xi$ is a finite set of all events. Its members handle all the possible transitions and they are the guards between all the states.

- $\quad \Sigma(s)$ is a set of feasible events at each state $s$. 
- $\quad$ rob is a set of transition probabilities. In probabilistic timed-automata different transitions between different states take place base on their assigned transition probabilities. The probabilities of transition between states are 1 if it is not mentioned on the guards.

According to the CSMA/CA protocol, transmission procedures freeze when the channel is sensed to be busy, and transmission is allowed to resume when the channel is determined to be idle again. After a busy period, according to CSMA/CA, each sender should sense whether the channel is idle for an AIFS period, then it should follow the procedure from the point at which it was stopped. A random backoff waiting time is used to avoid collisions as much as possible.

Figure 2-12 shows the proposed probabilistic timed-automata model for the sender of vehicle $j$. The initial location is indicated by a double circle. If the channel is sensed to be free (idle during the AIFS time period), the model jumps to the backoff state and randomly selects a backoff number from a uniform distribution (the contention window size is $C W=16$, so the probabilities are $1 / C W)$. After passing each slot time, the backoff counter counts down until it reaches zero. The events free and busy, which are generated by the channel, are urgent events for the Sender. Note that the Model moves toward transmission of the information by jumping to the vulnerable state. The vulnerable state and its clock timer are defined to model the time taken for sender to assess the channel and deliver its state to the MAC layer and switch from receive mode to transmission mode. Before switching to the vulnerable state, the sender was listening to the channel in order to determine whether the channel becomes busy, in which the procedure will be stopped. When the clock of vulnerable state reaches its final value, the model generates $\operatorname{Send} \hat{x}_{j}(k)$ messages for channel and fires the transmission timer in the transmit state.

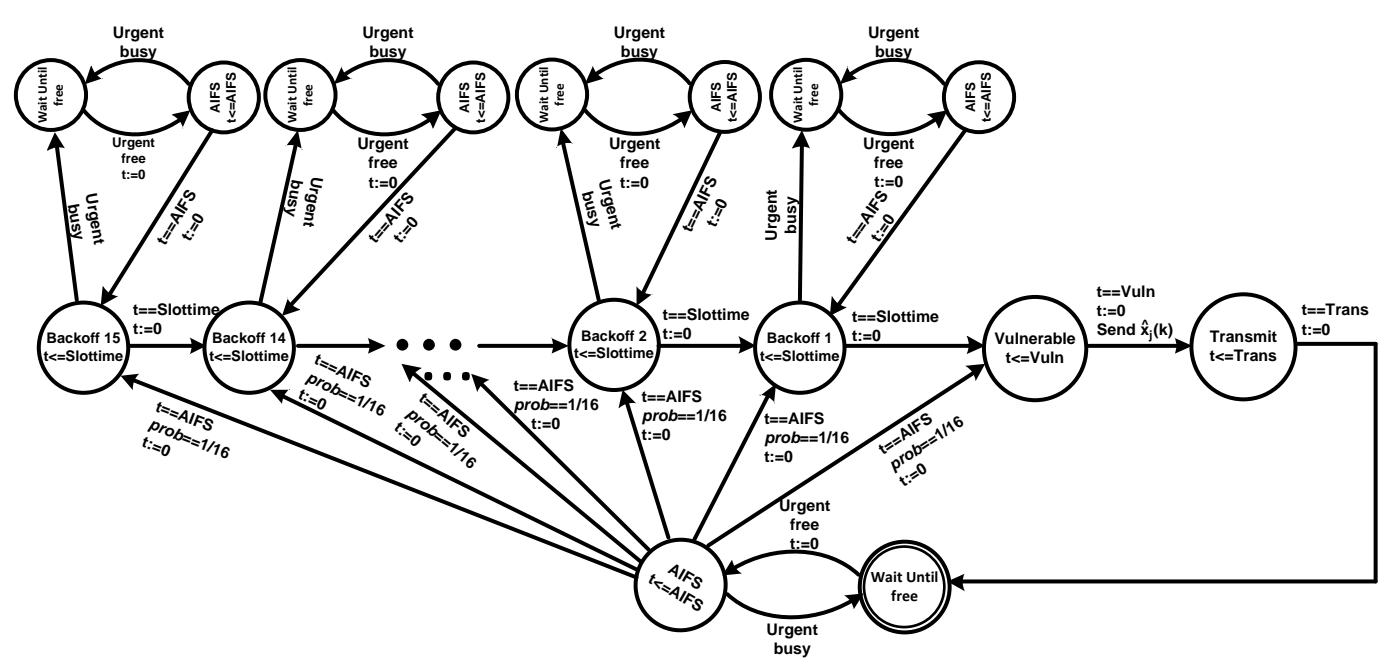

Figure 2-12- Probabilistic timed-automata model for sender at vehicle $j$ in the saturation condition

Trans value is chosen to model the time taken to transmit the information through the channel in the real word. Following a transmit state, the sender restarts the process. This assumes there is always information to transmit i.e., the node and network are in saturation mode. We make this assumption here to develop the basic model, but can relax it when the estimation process is also modeled. The hybrid model is described without the saturation assumption, because the sender 
should send the state information when it is necessary based on the real-time situational awareness estimation process policy.

Figure 2-13 shows the timed-automata model for the channel. We consider two states for the channel, busy and free. Note that the event Send $\hat{x}_{i}(k)$, generated by the transmitter of each vehicle, is an urgent event for channel. When a transmission happens (one sender leaves the vulnerable state), the channel jumps to busy state and delivers its state to all vehicles by busy massages. As soon as the sender finishes the transmission, the channel returns to free state. Therefore, in the case of all vehicles in range, they always have the same sense about the channel state (because they receive the same free or busy messages from the channel), but when there is a limited range of the transmission vehicles may have different sense about the channel state at different locations and this will yield to hidden node collision. If other transmissions happen while the first transmission has not yet completed and is still underway (this phenomenon could take place because of the vulnerable sate) the channel should wait in busy state until the newest transmission finishes. We model this notion with a self-transition on the top of busy state, which resets the busy clock.

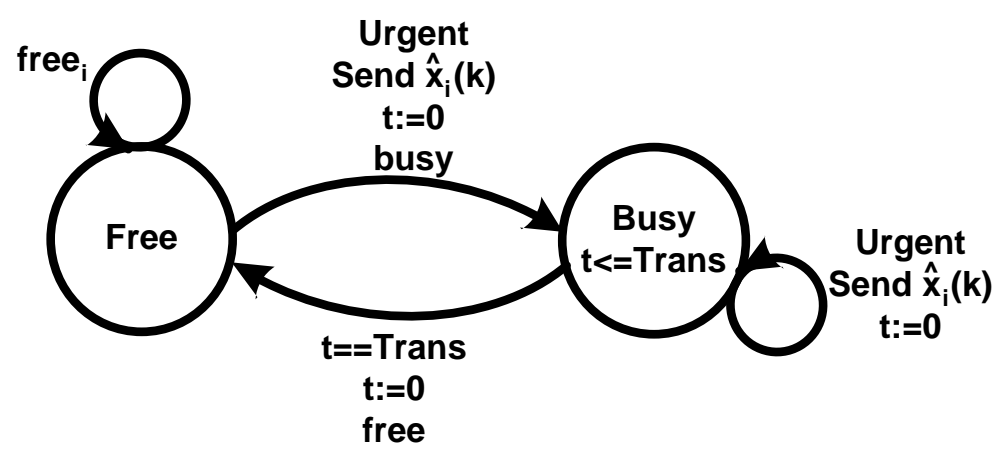

Figure 2-13 Probabilistic timed-automata model for shared channel.

Figure 2-14 shows the proposed timed-automata model of the receiver. We consider three states for the receiver to model information packet reception. When a transmission happens and the channel switches to the busy state, the receiver switches from waiting (while there are no transmissions on the channel) to the reception state. Note that the events $\operatorname{Send}_{i}(k), \operatorname{Sends}_{i}$ and free, generated by the transmitter of each vehicle and the channel model, are urgent events for the receiver too. It is also possible for multiple transmissions to occur at the same time $\left(\operatorname{Sends}_{i}\right)$. Therefore, we consider a transition for this condition to model these cases as collisions. If other transmissions occur while the first one is in progress, the receiver jumps to the collision state. After all of these events, the receiver model will go back to the waiting state when the channel sends a free message. The double circle indicates initial state of the receiver. 


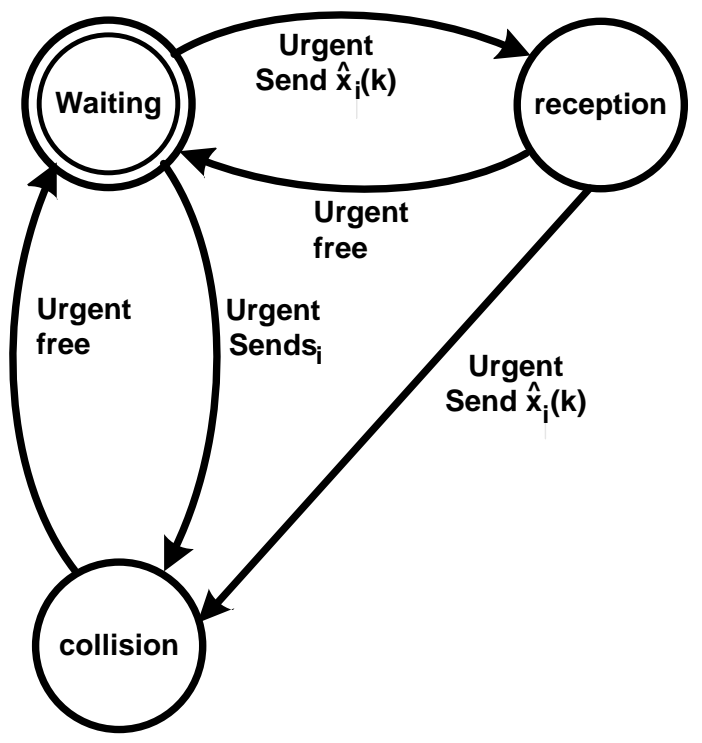

Figure 2-14 Timed-automata model for receiver part at vehicle.

One of the measures to verify a timed-automata model is reachability analysis, which indicates that the model will terminate in finite time in each independent broadcast of the information.

Problem 1 (Reachability): Given a transition system $T=\left(S, \Sigma, S_{0}, S_{f}\right)$, any state $S_{f} \in S_{f}$ is reachable from a state $s_{0} \in S_{0}$ (initial state) by a sequence of transitions.

Proof of state reachability: It was shown that each timed-automaton is a transition system. If we apply the following reachability algorithm presented in [133]:

\section{Initialization}

$$
\begin{aligned}
& \text { reach }_{0}=S_{0} \\
& \text { reach }_{-1}=\varnothing \\
& i=0
\end{aligned}
$$

while reach $_{i} \neq$ reach $_{i-1}$ do

begin

$$
\begin{aligned}
\text { reach }_{i+1}=\text { reach }_{i} \cup\left\{s^{\prime} \in S: \exists s \in \text { reach }_{i},\right. \\
\\
\left.\sigma \in \Sigma(s) \text { with }\left(s, \sigma, s^{\prime}\right)\right\}
\end{aligned}
$$

$$
i=i+1
$$

end

It is trivial to show that the proposed timed-automata model will terminate this algorithm after six steps, and reach $_{5}=S$. Therefore, the timed-automata is finite.

It can be concluded from this model that there are two reasons for collision when all vehicles are considered to be within range. First, if multiple transmissions occur at the same time (they have 
the same backoff number). Second, if two or more transmissions occur in sequence with vuln time duration (the difference between their backoff numbers is exactly equal to the vuln time slots). With very small vuln time slot, this type of collision can be ignored.

\subsubsection{Modeling the Estimation Process}

There are different policies to decide for communicating the information. It was shown that in the problem of tracking a dynamical system over a network if message generation and communication have a correlation with estimation error, the same performance as the periodic sampling and communication method can be reached using a lower rate of data [26, 27]. The reduced rate of transmission, without sacrificing performance, is a key advantage of the "error-dependent policy". In error dependent policy, each vehicle communicates its current state information (position and speed) only if the difference between its next position and what is perceived as its next position at the remote vehicle (remote estimator data) is greater than an error threshold. The next position of each vehicle is driven by a Kalman filter based on its current position (from GPS data), speed, and heading [134]. It was proved that a first-order kinematic model is rational for estimating a vehicle's updated position based on position and speed data already available to remote vehicles.

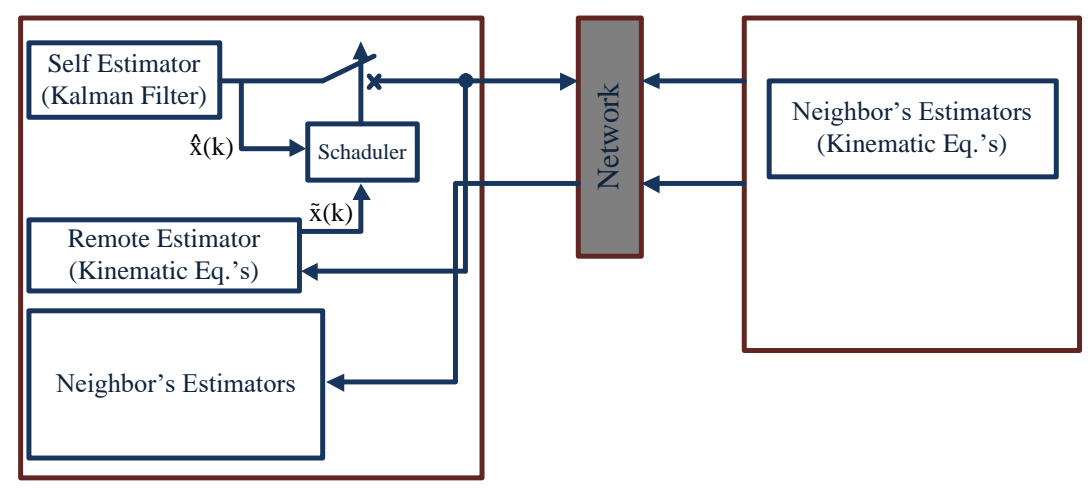

Figure 2-15 Schematic overview of Error-Dependent policy.

Figure 2-15 shows a schematic view of the estimation process. The remote estimator replicates the process running in other vehicles. It stores the last state information presented to the network to decide when the next transmission should occur. Self-estimation determines the next position based on a defined Kalman filter and generates a reference state vector. The remote estimator uses a first-order kinematic equation to estimate the next position of the vehicle based on state information broadcast in the previous transmission:

$$
\tilde{x}(k)=\tilde{x}(k-1)+\tilde{v}(k-1) \times \Delta T
$$

Symbols with $\left({ }^{\wedge}\right)$ on top refer to the Self Estimator's approximations and symbols with $(\sim)$ on top refer to the perceived or emulated Remote Estimator's approximations. The Scheduler decides whether the difference between the predefined reference position $(\hat{x}(k))$ and estimated position has reached the threshold error or not. It calculates the estimation error based on the next position measured by the Self Estimator and Remote Estimator, then compares its Euclidean norm with the estimation error threshold as soon as it receives it. If the error calculated by the scheduler, $e(k)=$ 
$\|\hat{x}(k)-\tilde{x}(k)\|_{2}$, is more than the estimation error threshold, $e_{t h}$, the current position and speed of the vehicle $(\hat{x}(k), \hat{v}(k))$ will be broadcast through the network.

\subsubsection{Hybrid Model}

The hybrid automata concept is a well-known method for mixed discrete-continuous state system modeling. The hybrid model proposed here to model the real-time situational awareness system is as follows:

$$
H=(Q, X, \text { Init }, \Sigma, f, \text { Dom }, E, G)
$$

Where $Q$ is a finite set of discrete states, $q_{1}$ represents the estimation process and $q_{2}$ represents the PTA model, $X$ is a set of continuous states. Any pair of $(q, x) \in Q \times X$ is referred as the state of $H . \Sigma$ is a set of discrete input symbols and events. Init $\subseteq Q \times X$ is the set of initial values of the system $\left(\hat{X}_{j}, \tilde{X}(0)\right) . f(\ldots): Q \times X \rightarrow p(X)$ represents the dynamic of the system. Dom: $Q \rightarrow$ $p(X) \times \Sigma$ is the working domain of each state and defines combinations of states, events and constraints (here error threshold) for which dynamical equations are allowed; here $p(x)$ is the power set of all subsets of $X . E \subseteq Q \times Q$ is the set of edges, and $G: E \times \Sigma \rightarrow p(X)$ is the set of transition guard conditions that enable the transition between discrete states.

Figure 2-16 shows the proposed hybrid model for CVS system for vehicle $j$. The estimation process $\left(q_{1}\right)$, estimates the next position of vehicle according to Equation (2.20). Scheduler determines the error of the estimations based on the information of Self Estimator and Remote Estimator and compares it with the Threshold error, and decides whether the transmission is necessary or not. The reference vector, $\hat{X}_{j}$, contains the real position and speed $(\hat{x}(k), \hat{v}(k))$ of each vehicle at different time steps (information of Self Estimator). If the estimation error is more than the Threshold error, the system will switch to the probabilistic timed-automata model and starts the communication process (probabilistic timed-automata model) from wait until free state. After that the system leaves the Transmit state of the probabilistic timed-automata, the system will update the Remote Estimator information (position and speed) with the current position of vehicle.

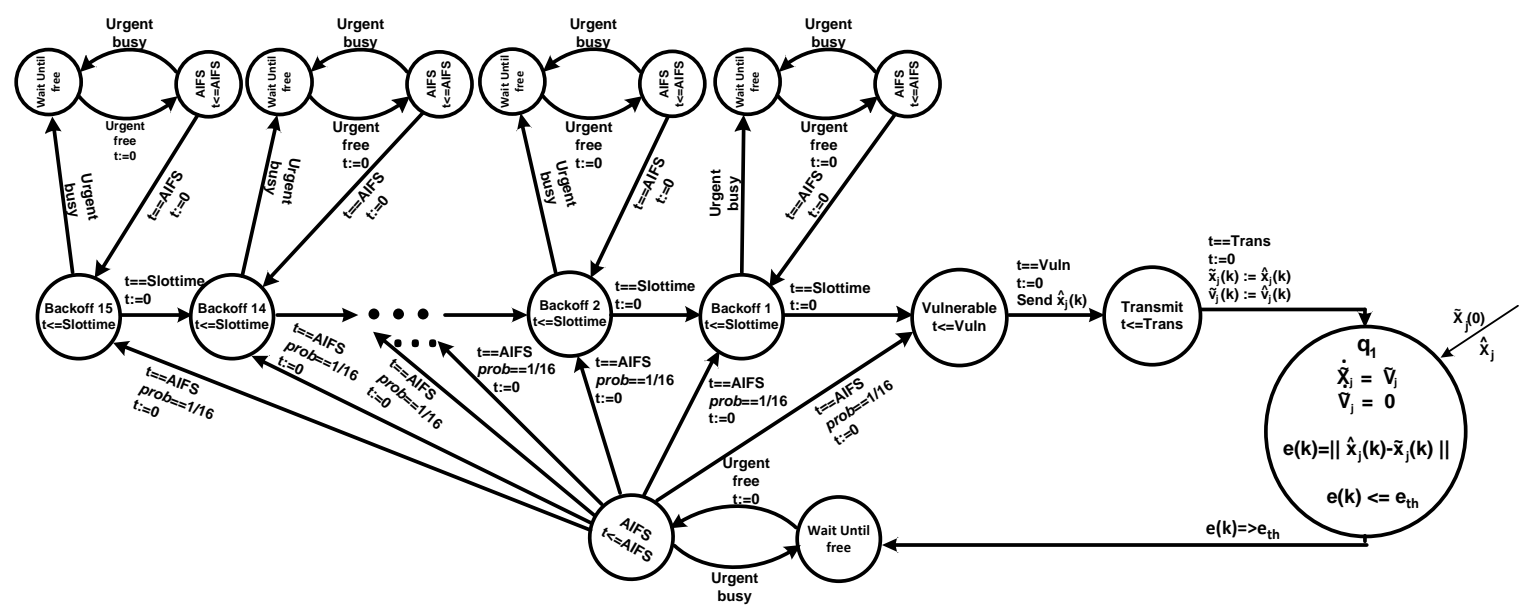

Figure 2-16 Hybrid model for CVS system at vehicle $j$. 
Lemma $2-1: \quad$ The proposed Hybrid model is non-blocking.

Proof: Based on the definitions the set of reachable state in continuous part (S1) is:

$$
\text { reach }=\left\{q_{1}\right\} \times\left\{\tilde{x}(k) \mid e \leq e_{t h}\right\}
$$

Since that the nature of the vehicle speed based on driver reactions is random (because of sudden breaking) we have two possibilities for Trans (transition guard) set, that are:

$$
\begin{aligned}
& \text { Trans }_{1}=\left\{q_{1}\right\} \times\left\{\tilde{x}(k) \mid e>e_{t h}\right\} \\
& \text { Trans }_{2}=\left\{q_{1}\right\} \times\left\{\tilde{x}(k) \mid e=e_{t h}\right\}
\end{aligned}
$$

If $\operatorname{Trans}_{1}$ is the case, it is trivial that Reach $\cap \operatorname{Trans}=\varnothing$ and thus the model is non-blocking. If Trans $_{2}$ is the case, it is obvious that Reach $\cap$ Trans $\neq \emptyset$. Considering the following lemma, because there is a guard between the estimation and communication process $\left(e \geq e_{t h}\right)$ therefore, the Hybrid model is non-blocking. Note that we proved that the communication process is finite timed-automata.

Lemma 2-2: $\quad$ A Hybrid automaton, $H$, is non-blocking if for all, $(q, x) \in$ Reach $\cap$ Trans there exists $\dot{q} \in Q$ such that $(\dot{q}, q) \in E$ and $x \in G(q, q)$.

Proof in [133].

\subsubsection{Modeling Hidden Node Phenomenon}

So far we have assumed a channel which sends free and busy messages to all vehicles; therefore, each vehicle has the state of the entire network. In the case of a limited range of transmission, vehicles are not able to broadcast information to all other vehicles, and only vehicles within a certain distance (communication range) of a transmitting vehicle will receive the transmitted data. It should be noted that in this case vehicles do not have the information of the entire network and they only can listen to the channel within their range. Figure 2-17 presents a simplified illustration. Consider a case where vehicle $\mathrm{B}$ has information to transmit and listens to the channel; if the channel is determined to be free, then vehicle $\mathrm{B}$ will attempt to broadcast its information according to CSMA/CA. However, vehicle D, which is not located within the range of vehicle $\mathrm{B}$, attempts to broadcast its data while vehicle B is transmitting, yet not violating CSMA/CA rules. In such a case, the packets from the two vehicles will collide at the receiver, vehicle $\mathrm{C}$, who is located between and within the range of both senders. These collisions could adversely affect the estimation process if the network is not properly managed. 


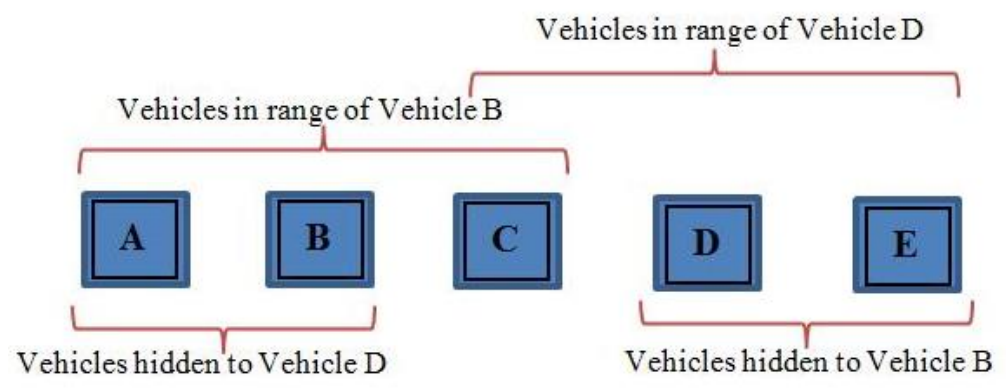

Figure 2-17 Schematic overview of hidden node phenomenon.

In the model of this case (limited range), each vehicle has the information of vehicles within its range, but not of the entire network. Therefore, the authority to make decisions is local. Figure 2-18 shows the proposed model for the receiver part of vehicles. In this model, we embed the channel model inside the receiver (because each vehicle senses the channel based on its range), and each receiver provides free and busy messages only for its transmitter. Each vehicle receives Send $\hat{x}_{i}(k)$ and Sends $s_{i}$ signals only from vehicles located within its range.

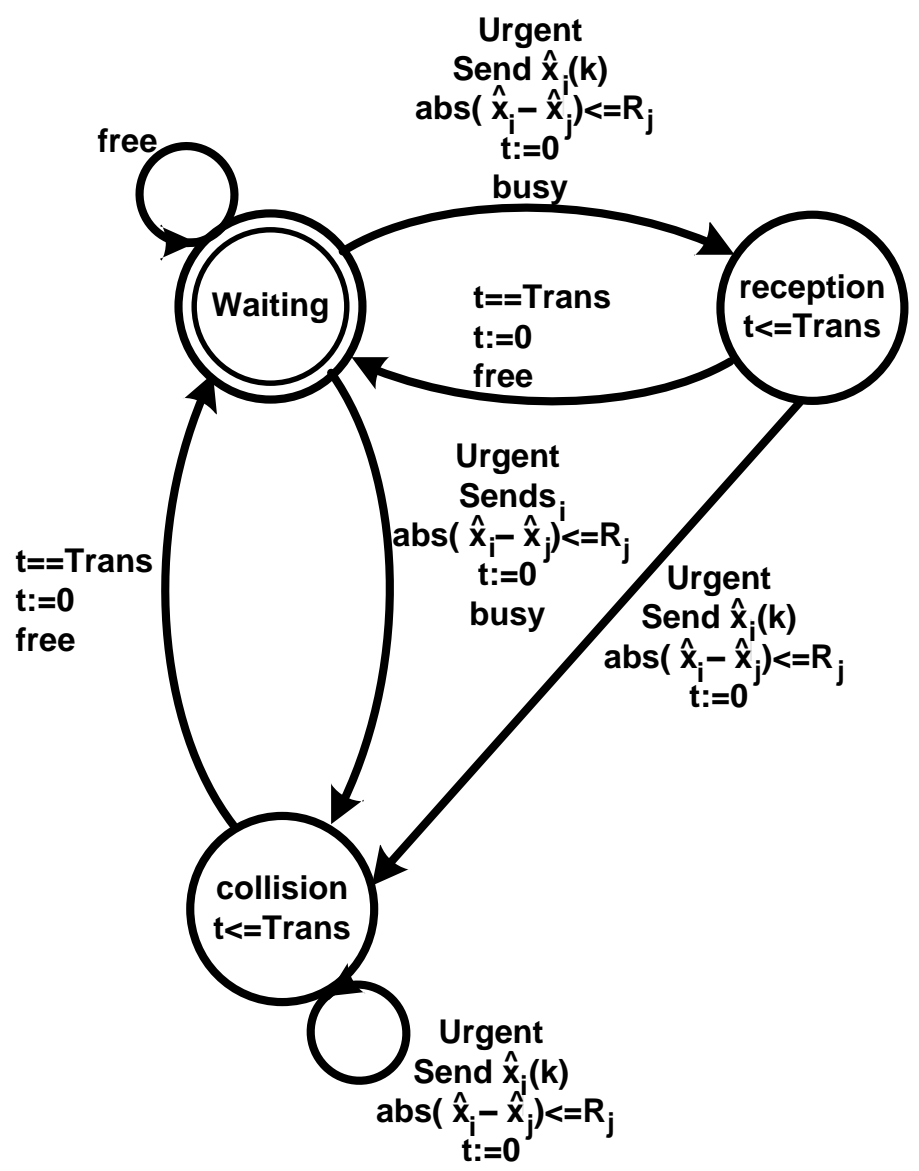

Figure 2-18 Receiver model for vehicle $j$. 
We define a closed sphere around each vehicle to indicate the range $\left(R_{j}\right)$. In this model of receiver, we have three states namely waiting, reception, and collision. In the waiting state, the receiver produces free messages repeatedly, allowing for data to be broadcast. The model for the sender and the hybrid model will be the same as before. Furthermore, because these free and busy messages are not shared with other vehicles, the hidden node collision will be inevitable.

\subsubsection{Model Verification}

To evaluate the accuracy of the model, we compare the results generated by the model and Network Simulator 3 (ns-3) for several different scenarios. We consider the probability of successful transmissions with respect to the number of vehicles, range, and rate of transmission as the measure for this comparison. The model is implemented in a multi-thread program in Java. We study the model for two cases of a fully-connected network (all vehicles in range) and hidden node scenario (in a highway setting).

The ns-3 is an open source discrete event simulator that is maintained by a large community of researchers and developers. It is currently in use by automotive industry for verification of vehicular communication and their related systems. Due to its discrete event nature and the fact that real-time situational awareness runs as a periodic (discrete) repetitive process, it is straightforward to implement all the relevant parts of real-time situational awareness in ns- 3 . To verify the proposed model, we configure ns-3 in a way that it behaves similarly to the model. To this end, an ideal channel was created with no fading and propagation loss. This means that each transmitted packet is successfully delivered to receivers up to a specified distance regardless of phenomenon such as fading. This distance shows the transmission range, thus the packet will not be received at any receiver beyond it. The PHY and MAC layer in ns-3 are configured to mimic DSRC (802.11p in $10 \mathrm{MHz}$ ) in broadcast mode (AdHoc) with a rate of $6 \mathrm{Mbps}$.

All clocks of models are scaled based on a fix slot time (time duration of $13 \mu \mathrm{s}$ ). Hence, other time-dependent model parameters (Table 2-1) are calculated based on this slot-time. The same calculation also is considered for ns-3. For example, one second of simulation time will be 76923 time slots, the packet size in ns-3 is 388 Bytes (324 payload+64 header).

Table 2-1- System Parameters

\begin{tabular}{l|r}
\hline \multicolumn{1}{c||}{ Parameter } & Value \\
\hline \hline Slot Time & $13 \mu$ sec \\
\hline AIFS & $2 \times$ slot time \\
\hline Vulnerable Time & $1 \times$ slot time \\
\hline Transmission time & $38 \times$ slot time \\
\hline Contention Window size & $16 \times$ slot time \\
\hline Packet size in ns-3 & 388 bytes \\
\hline Channel Capacity & 2026 packets \\
\hline \hline
\end{tabular}

Starting the CSMA/CA procedure from sent state instead of a pre-start back-off is the significant implementation issue of ns-3, which causes a completely concurrent collision in all of the transmitted packets. To avoid this issue, each vehicle in ns-3 should initialize its transmission by 
choosing a random delay drawn from a uniform distribution with the size of the contention window.

\subsubsection{Fully Connected Network Scenario}

The probability of successful transmission is calculated by averaging over the number of successfully received packets (the number of times the reception state was visited subtracted by the number of times the collision state was met by a receiver) divided by the number of transmitted packets.

Figure 2-19 depicts the probability of successful transmission according to various numbers of vehicles for constant transmission rate $(50 \mathrm{~Hz})$. As it was expected, the probability of success decreases after the channel becomes saturated.

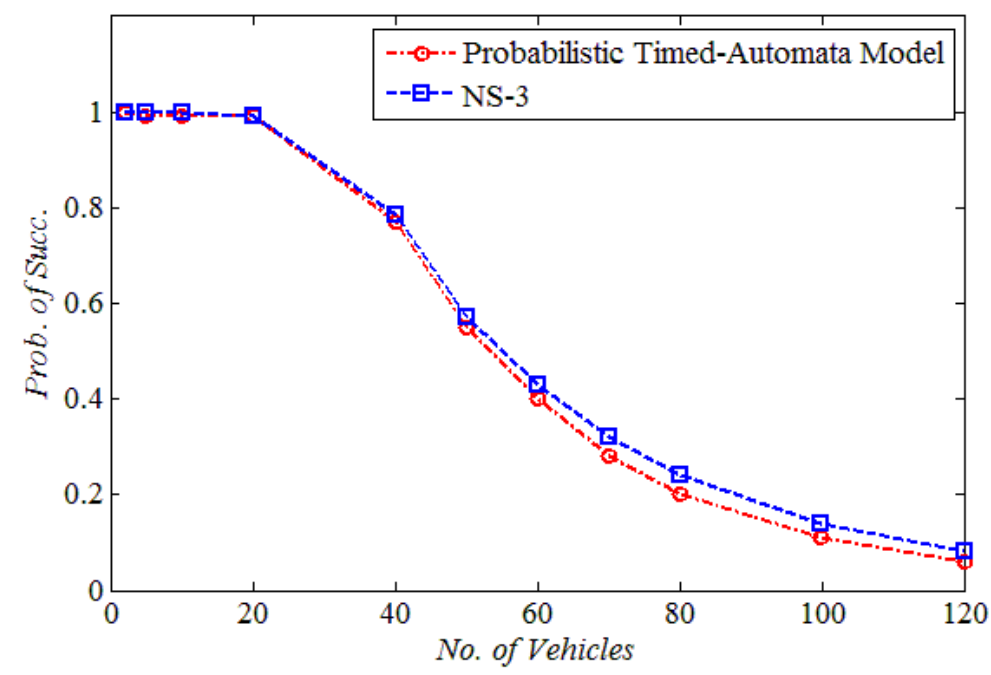

Figure 2-19 Probability of success vs. number of vehicles - all in range scenario.

The probability of successful transmission for different rates is shown in Figure 2-20. The results are shown for three different cases of 40, 50, and 60 vehicles. The channel behavior can be observed while it shows a saturation point for each case, which depends on the number of vehicles, and a unique pattern of decreasing. 


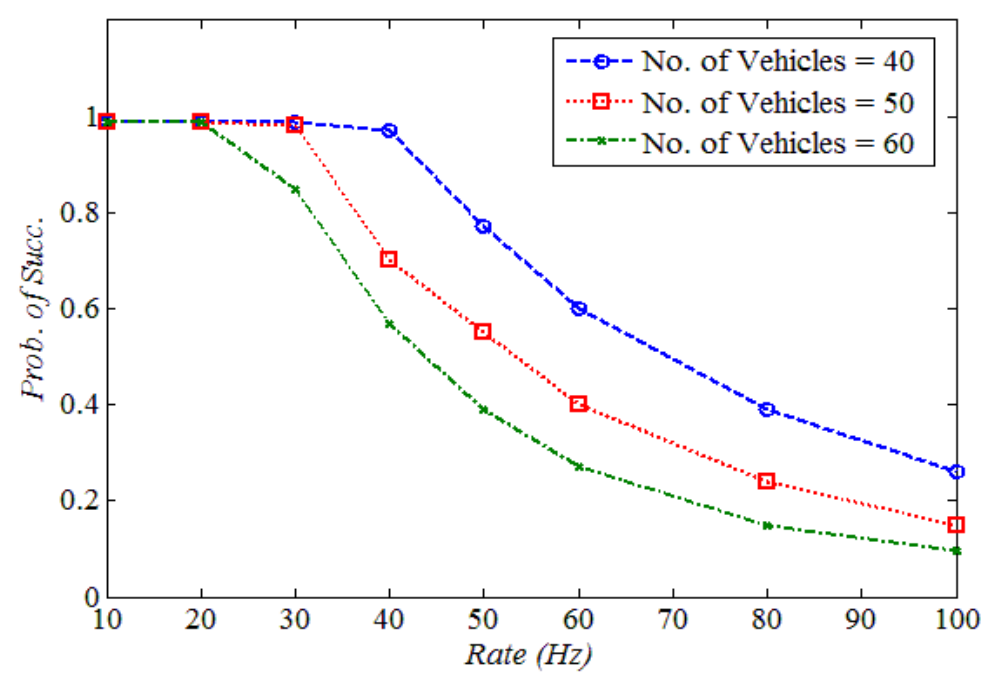

Figure 2-20 Probability of success vs. transmission rate - all in range scenario.

\subsubsection{Hidden Node Scenario}

In this scenario, vehicles are distributed in a highway-like pattern. To investigate the effect of hidden node problem precisely, and avoid effects of edges of the topology, the middle $1 / 5$ of the vehicles are used as the test nodes to calculate the probability of successful transmission (e.g. 50 vehicles in the center out of a highway with 250 vehicles). ns- 3 uses the location of vehicles to create a mobility trace file. The proposed Hybrid model also uses the same reference vector of positions for its estimation process $\left(\hat{X}_{j}\right)$.

Figure 2-21 shows the probability of successful transmission for hidden node scenario with respect to different ranges of transmission. Note that the rate of transmission is fixed to $10 \mathrm{~Hz}$ and the values are calculated over 50 vehicles in the center of a highway with 250 vehicles. As the range of transmission increases, the average probability of successful transmission in a neighborhood around vehicle increases; the reason is that more vehicles are aware of each other's transmission and cooperate with each other in a CSMA manner. It must be noted that the total capacity decreases in this case since the channel is shared by more vehicles when the range of transmission increases. It is only the probability of success for the few transmitted packets that increases. 


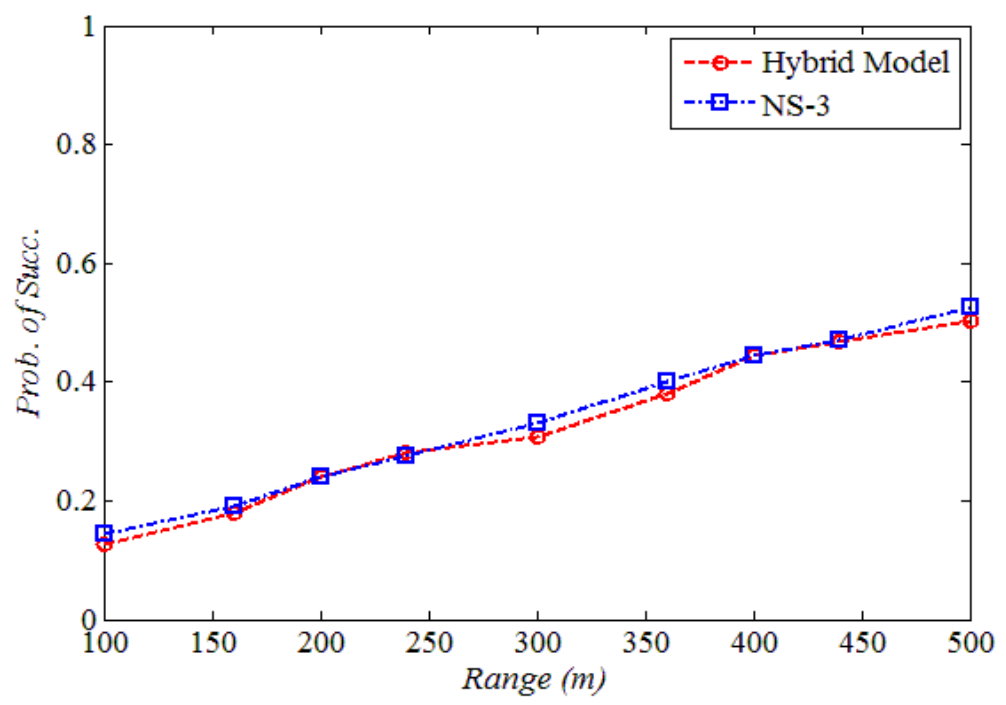

Figure 2-21 Probability of success vs. transmission range - Hidden node scenario.

The mutual effect of transmission rate and range on successful transmission in the case of 50 vehicles is shown in Figure 2-22. Since the capacity of the channel is limited to a constant amount of packets, increasing the rate will improve the probability of successful transmission until it reaches the saturation point of the channel. After that, increasing the rate will increase the number of hidden node collisions which results in less successful transmissions.

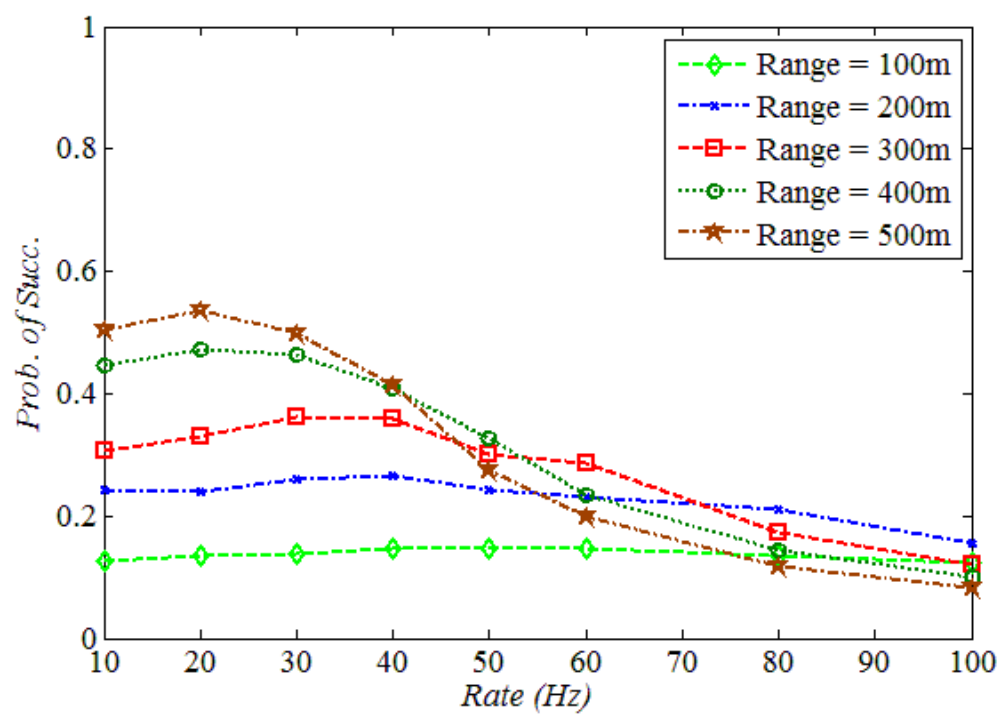

Figure 2-22 Probability of success vs. transmission rate and range - Hidden node scenario.

Another measure used to evaluate the Hybrid model is the rate of message transmission as a function of the estimation error threshold. The reference position vector, $\hat{X}_{j}$, is assumed to perform 
like a GPS device, which updates the speed of the vehicles every $6.5 \mathrm{msec}$ via a zero mean Gaussian distribution for acceleration. Therefore, every $6.5 \mathrm{msec}$ the speed vector will update its value.

Figure 2-23 shows the result for different values of threshold error. It can be observed that the increase of the error threshold affects the rate of transmission inversely (as expected).

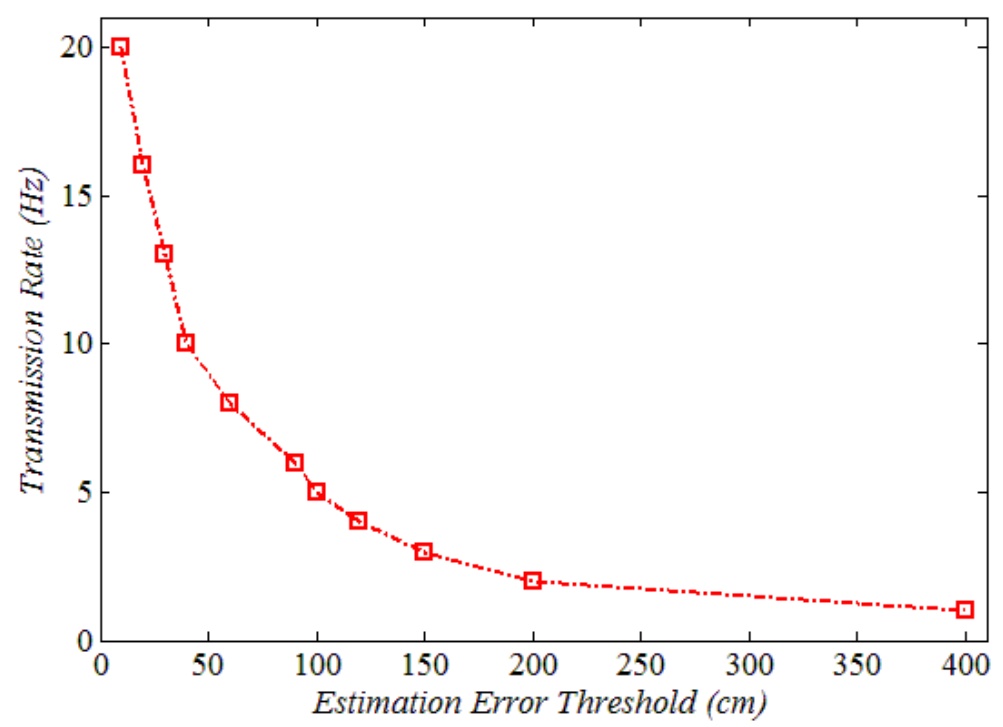

Figure 2-23 Effect of threshold error on transmission rate. 


\section{Chapter 3 Multi-hop Situational Awareness through Network-aware Double-layer Distance-dependent Broadcast}

Intelligent transportation systems (ITS) for vehicles are categorized into three major groups based on their application: comfort/entertainment, efficiency, and safety. While the communication requirements of the comfort applications can be met by traditional technologies such as cellular networks, efficiency and safety applications need a communication infrastructure with lower latency and higher capacity. Vehicle-to-vehicle (V2V) communication (enabled using dedicated short range communication (DSRC) [42]) has emerged as an appropriate platform for such applications. DSRC is a promising technology to be used for creating single-hop and multi-hop networks. Single-hop communication is mostly used in safety applications with strict latency requirements; for example, collision-avoidance systems need real-time awareness of their surroundings. Multi-hop communication is more useful for applications that need to disseminate vehicle or road information to distances much farther than a single-hop transmission range. Use cases for multi-hop communication include both safety applications such as emergency vehicle warning and post-crash warning, as well as efficiency applications like traffic information, vehicle tracking, traffic management, and road monitoring. Due to the vital role of time-critical safety applications, single-hop communication has attracted more attention in the research community. On the other hand, multi-hop communication has mostly been studied for generic applications, without much attention to the specific needs and features of efficiency and safety systems. The problem that we focus on this chapter is the coexistence of critical safety information along with multi-hop communication of general traffic and less critical information.

Most multi-hop safety and efficiency applications rely on information such as traffic congestion, car density, movement dynamics, or road blocks, whose importance depends on how far the recipient is from the source of information. In these applications, as they have a reasonable tolerance for delay, immediate forwarding of the information to very long distances is not beneficial. As a result, a desired information forwarding method would be one that reduces the amount of forwarded information as the distance from the source increases to preserve valuable 
resources. Thus, in this chapter, we pursue this goal by proposing a network-aware double-layer distance-dependent broadcast protocol (N2DCAST). Our protocol ensures that important information is delivered to its point of interest with small latency without overwhelming scarce network capacity.

Broadcast of information in vehicular ad-hoc networks (VANETs) requires strategies to avoid congestion in the communication channel. The rate, range, and message size of transmissions are the most influential factors on channel congestion. A large body of literature exists on determining such parameters for single-hop broadcast (e.g., [27, 32]), yet few solutions have been proposed to address this issue in the multi-hop broadcast. These solutions are based on the two competing variants of beaconless independent transmissions or piggybacking over single-hop beacons [135]. All of these solutions mostly aim to find optimal forwarding procedures with the objective of minimizing redundant rebroadcasts, contention, and collisions. However, our proposed method targets the reduction of latency in consideration of channel load.

In practice, systems that will be designed based on the assumption of existing single-hop beacons are more feasible to become a reality. For example, piggybacking over V2V basic safety messages (BSMs) eliminates the need for creating a whole new high-penetration network. V2V communication is expected to be implemented through government mandates in the next few years [87]. Whereas other types of high-penetration networks created by voluntary participation of vehicles may not succeed in the near future. Even though piggybacking on beacons is not the fastest way of forwarding information, it has the lowest impact on network load because it avoids the overhead of creating new packets. Specifically, the study of the impact of piggybacking on forwarding delay, reception probability, and staleness probability of the received packets, has found that the relationship between increasing the size of piggybacked data and forwarding delay is logarithmic [44]. Furthermore, it has been shown that the channel load is reduced when piggybacking over beacons is used instead of increasing the coverage of single-hop beacons [136].

The contribution of this chapter is a multi-hop broadcast protocol based on piggybacking of aggregated traffic information over BSMs. We enhance our previous distance-sensitive method [137] and append a new distance-dependent forwarding algorithm to it. The forwarding algorithm uses two layers of distance-dependency. The first layer uses a message size control scheme and a mix probabilistic approach to ensure that the forwarding within each hop is as fast as possible. The mix probabilistic approach incorporates network-awareness into the forwarding algorithm. Therefore, packet loss in the network and importance of information is considered in the protocol. The second layer progressively decreases the forwarding rate of subsequent hops as the distance from the source increases to preserve channel load. Directional broadcast of messages, based on application requirements, is achievable by this protocol as there is no assumption or limitation exists over movement directions. The proposed method considerably reduces the overall staleness of information and channel load. Additionally, if the same amount of channel load is utilized as in similar approaches, whenever the network is not threatened by congestion, this method increases the coverage of traffic map.

The rest of this chapter is structured as follows. Section 3.1 describes the system design with a detailed description of the proposed protocol. The formal protocol specification is presented in Section 3.2. The performance evaluation and experimental results are discussed in Section 3.3. 


\subsection{System Design}

In this section, we first motivate our approach and describe the system model; then, the proposed schemes and algorithms are presented, followed by an analysis of their characteristics.

\subsubsection{Performance Metrics}

In general, each broadcast method is desired to have four characteristics: reliability, reachability, minimum delay, and scalability. Reliability and reachability describe how frequent vehicles receive a message containing traffic or safety information and how far (number of hops) the message reaches. Reception rate and delivery ratio are the measures associated with reliability and reachability, respectively. In the definition of reliability and reachability, the delay by which a message reaches an intended receiver is not a factor, as in some applications the information dissemination is better late than never. Latency and staleness are the performance metrics associated with delay for time-critical applications. Each forwarding step has a latency which adds a delay to the message until it reaches the destination. Staleness of a message is equal to the difference between the current timestamp of the receiver and the generation timestamp of that message with respect to a unique time reference. Apart from these three characteristics, scalability shows the ability of the protocol to handle the high load on the channel and can be measured by overhead, forwarder ratio, and channel load.

\subsubsection{Reliability and Reachability}

Given a fully connected network and the existence of frequent single-hop safety beacons (BSMs), all the methods that rely on piggybacking over these beacons are capable of achieving 100-percent reachability. For example, in the counter-based broadcast method when a vehicle broadcasts a message, all the vehicles that receive this message count the number of times they heard the rebroadcasted message. Accordingly, if a vehicle hears the message for the first time, it will set the counter value to one, and if it overhears the same message, the counter will be incremented by one. On the other hand, if the message is not heard between the duration of two consecutive beacons of the vehicle, the counter will be decremented to zero and the message will be retransmitted. Consequently, any mechanism that safeguards the retransmission of a message by at least one vehicle at each hop, can guarantee the spread of that message through the whole network as it is fully connected and the beacons are generated periodically [138]. In our method, each vehicle embeds the received message in its next beacon based on a probability. Therefore, to achieve the same reachability and reliability, the probability of success is defined in a way that ensures the messages are rebroadcasted by at least one vehicle for each beacon period, while the reliability factor of the network is also considered and predetermined (see Figure 3-1). Thus, the expected value of rebroadcasting a message is always greater than one. 


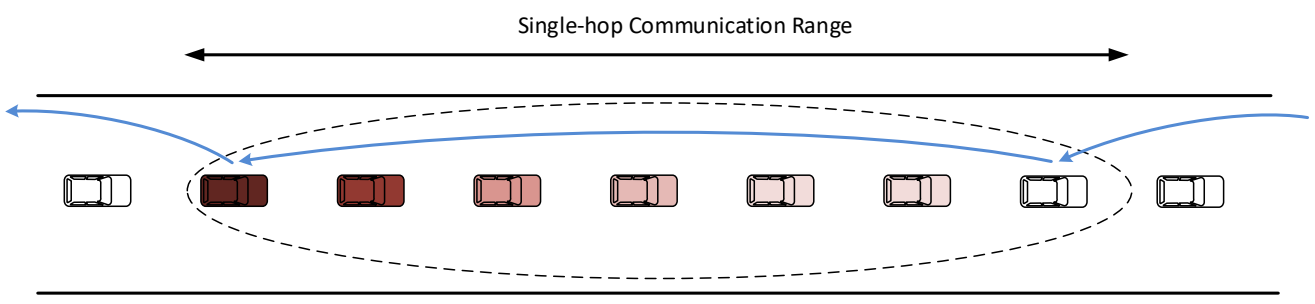

Figure 3-1- Desired multi-hop forwarder

\subsubsection{Scalability}

Since piggybacking over existing beacons is used in our method, overhead is not a proper metric for the scalability measurement since no new packets are generated by our method. Thus, forwarder ratio and channel load are used for this aspect. Forwarder ratio is defined as the ratio of a number of vehicles which rebroadcast a message divided by a total number of vehicles in a single-hop communication range. In general, channel load shows the amount of load put on the channel due to rebroadcast of a message, while in our method channel load means the "added load" due to attaching of multi-hop messages to BSM packets. Moreover, the amount of channel load can affect the performance of any method in terms of staleness and reachability. As it is shown in [32], the information dissemination ratio (IDR) increases with an increase in the channel load up to a peak point, after which it sharply decreases. This finding is a consequence of the fact that putting too much load on the communication channel leads to a high packet loss, which results in an increase of staleness and reduction of reachability. As a matter of fact, if the channel load was not an issue, flooding, which is the most primitive multi-hop broadcast method, would have been the best option. In flooding, each vehicle rebroadcasts every received message immediately, which would have resulted in the highest reachability and lowest possible staleness if no packets were dropped due to the channel congestion [138]. Therefore, a desirable method should not only have a high reachability and low staleness but should also maintain the channel load around the peak point at which the IDR is maximized.

To investigate the effect of our proposed method on channel load, we should first determine the network parameters that affect channel load. As mentioned before, the rate, range, and message size of transmissions are the most influential factors on the channel load. Let $C$ denote the channel capacity (in bits per second) and $r$ denote the rate of transmissions (in Hertz). Furthermore, denote the single-hop communication range (in meters) by $D\left(P_{t x}\right)$, where $P_{t x}$ is the transmission power. The number of vehicles in the communication range of a specific vehicle can be calculated as $N=$ $2 \times\left(\rho \times D\left(P_{t x}\right)\right)$, where $\rho$ is the vehicle density observed by that specific vehicle, defined as the number of vehicles per unit distance in a unidimensional network. Please note that for simplicity of notation and analysis, we are not showing the vehicle identifying subscript of $\rho$ and $N$ in following equations. In a scenario where all the vehicles are in the communication range of each other and use the same parameters, the channel capacity should always satisfy the following condition

$$
C \geq \ell \times r \times N
$$

where $\ell$ is the packet length (in bits) containing the BSM and other piggybacked data. 
Since our method uses piggybacking over single-hop beacons and it is orthogonal to the singlehop safety application, it has no control over the rate or range of the transmissions and they are adjusted by the single-hop safety algorithms. However, the message size can be controlled by our method since each packet contains BSM along with some traffic data units (TDUs). The aggregated traffic information of a hop is referred to as the traffic data unit (TDU). The number of TDUs to be included in each packet is determined by our message size control scheme.

According to the IEEE 802.11p standard, the payload size of a packet is limited to 18,336 bits. However, the payload size of a packet, as found from (3.1) for the given channel capacity, is always less than the standard. In consideration of the fact that packet fragmentation should be avoided as it significantly affects the channel load and congestion. Additionally, the packet length as determined from (3.1) is based on the assumption that the full accessible channel capacity can be utilized. As mentioned before, to maximize the IDR in a CSMA/CA broadcast network, the actual channel load should always be less than the channel capacity. Therefore, the actual packet length supported by such networks should be less than the value of $\ell$. Hence, to avoid channel congestion due to large packets, our message size control scheme uses only a portion of the channel capacity and embeds a certain number of TDUs to each packet.

\subsubsection{Staleness}

As described before, staleness of the state of vehicle $j$ at vehicle $i$ at any given time is the elapsed time from the generation time of the latest received message about vehicle $j$, at vehicle $i$. Note that when new information about vehicle $j$ is received, the staleness at that time equals the latency of delivering the message. Staleness in the state continues to increase until the next message from vehicle $j$ is received. The maximum staleness thus depends on both the source broadcast rate as well as the forwarding latency. This is the staleness value that is reported in our analysis and evaluations. The forwarding algorithm in our protocol ensures that the staleness of the information which is delivered to its point of interest is low, taking into consideration the channel load and network conditions to avoid congestion.

\subsubsection{Algorithm Description}

\subsubsection{Message size control scheme}

As it was mentioned earlier, our message size control scheme uses only a portion of the channel capacity for TDUs. This portion is defined as the channel capacity utilization ratio and denoted by $\beta(0 \leq \beta<1)$, which results in the following packet length:

$$
\ell^{*}=(1+\gamma) \times\left(\ell_{\mathrm{BSM}}+m \times \ell_{\mathrm{TDU}}\right)
$$

where $\gamma$ is the ratio transmission overhead due to the required packetization (i.e., headers, CRC, security, etc.), and $m$ is the maximum allowed number of TDUs to be embedded in a packet. The value of $m$ is dynamically recalculated before each beacon and can be found as

$$
m \leq \frac{\beta \times C}{r \times N \times(1+\gamma) \times \ell_{\mathrm{TDU}}}
$$

where $\ell_{T D U}$ is the length (in bits) of a TDU message. 
The content of each TDU is determined based on the requirements the multi-hop safety and efficiency application. For example, in a traffic information application, each TDU consists of Timestamp, Position Information, Traffic Density, Velocity, Lane Density, and Congestion Condition of its corresponding region. However, the only information that is required by our protocol is timestamp and position information of the original region.

Although each vehicle can transmit at most $m$ TDUs, it can receive and store much more TDUs to be rebroadcasted. Nevertheless, not every vehicle needs to retransmit every TDU. Even if a vehicle should retransmit a TDU, the retransmission can be performed with a delay, based on the level of tolerance of the application. Therefore, the decision of which TDU and the time it should be retransmitted is extremely important and significantly affects the staleness.

\subsubsection{Forwarding algorithm}

The forwarding algorithm uses two different distance-dependent schemes.

\subsection{Inter-hop distance-dependent scheme}

Let $d(i, j)$ and $d_{h o p}(i, j)$ denote the Euclidian distance and hop distance between vehicles $i$ and $j$, respectively, where the hop distance is calculated as

$$
d_{h o p}(i, j)=\left\lfloor\frac{d(i, j)}{D\left(P_{t x}\right)}\right\rfloor
$$

As the importance of traffic information in multi-hop broadcast is dependent on how far the recipient is from the source, rebroadcast of every received TDU is unnecessary. The forwarding rate of subsequent hops should progressively decrease as the distance from the source increases.

Forwarding rate of $\widehat{R_{h}}=\frac{1}{h}$ where $h=d_{h o p}(i, j)$, is used in our previous distance-sensitive method for vehicles that are $h$-hops away. However, this rate can be adjusted based on time requirements of the applications. Only one condition should always be held, which is expressed as

$$
\widehat{R_{1}} \geq \widehat{R_{2}} \geq \cdots \geq \widehat{R_{h}} \geq \cdots
$$

It has been proved in our previous work that the order of complexity of the forwarding rate per vehicle with the distance-dependent scheme is always smaller than $O(N)$, which is the complexity of forwarding without distance-dependency [137].

\subsection{Intra-hop distance-dependent scheme}

To describe the significance of the proposed scheme, it is firstly required to determine the effect that next forwarding vehicle inside the single-hop communication range has on staleness. To give an example, in counter-based rebroadcast method, consider a situation in which $k$ vehicles are following each other and they are all in single-hop communication range of each other. Assume the leading vehicle (i.e., vehicle number one) receives a message from the adjacent hop and rebroadcasts it. Upon the reception of the message, all $(k-1)$ remaining vehicles should decide whether to rebroadcast this message or wait for others to do so. Subsequently, it makes a big difference whether the last vehicle in the row or the vehicle next to the leading vehicle rebroadcasts the message. When the last vehicle relays the message, information is disseminated to a far distance after it, but when the vehicle next to leading vehicle relays the message, this dissemination 
only covers a short distance farther than the previous relay. Staleness consequently increases in the later situation since this rebroadcast suppresses the rebroadcast of other neighboring vehicles.

It can be inferred from the above example that ideally, it is optimal to let the farthest vehicle in communication range to take the responsibility for next retransmission. However, even if a vehicle knows other vehicles exist after it in a communication range and suppresses the rebroadcast for those vehicles to send out the message, they might not even get the message as packet error ratio (PER) always increases by distance. Hence, the next forwarding vehicle should take into account not only the distance from the first transmitter but also the probability of successful reception of the message by further vehicles. The following distance-dependent probabilistic approach is proposed to achieve both objectives.

Since the network conditions are changing and could be different for each vehicle, vehicles should individually decide whether to be the next forwarder or not. This decision making happens probabilistically. Whenever a vehicle receives a message, if the message is received for the first time, a Bernoulli trial with a predefined success probability, will determine whether the message should be rebroadcasted or not. For instance, in a uniform probabilistic rebroadcast method, the success probability is the same for all vehicles. Therefore, each vehicle $i$, independently conducts a Bernoulli trial with success probability of $p_{i}=\frac{\delta}{N}$ to decide whether to forward a message or not, where $N$ is the number of vehicles in a single-hop communication range and $\delta(1 \leq \delta \leq N)$, which is preset based on the requirements of multi-hop safety or traffic application, is defined as the reliability factor of the approach. Taking into account the fact that a higher $\delta$ means a higher chance of rebroadcast because more vehicles decide to include the message as the success probability is equal for all vehicles, $p_{1}=\cdots=p_{i}=\cdots=p_{N}=\frac{\delta}{N}$. Thus, higher $\delta$ leads to increase of the forwarder ratio and channel load.

Even counter-based rebroadcast method implicitly uses the same uniform probabilistic approach as each transmitter in CSMA/CA has to pick a back-off counter based on 802.11 standard for MAC layer. The back-off counter is randomly chosen from a uniform distribution between zero and the size of contention window; while the number of competing transmitters would be the optimal value for the size of the contention window. Therefore, a probabilistic method with the correct choice of the reliability factor is capable of replicating the behavior of counter-based approach, which is a deterministic method and achieves the same staleness and channel load.

In contrast, in our proposed probabilistic distance-dependent approach the success probability of Bernoulli trials are not equal for all vehicles. The success probability of a Bernoulli trial in vehicle $i$, to determine whether to include the received message from vehicle $j$, depends on the distance between two vehicles and the value of PER that has been maintained for that distance. The PER represents the ratio of the number of missed packets to the total number of packets expected to be received during every PER interval. The PER is a local measure calculated using the sequence number contained in each BSM in a receiving vehicle $i$ for a transmitting vehicle $j$, and is denoted by $P E R_{i}(j)$. Recording the received sequence numbers indicates how many packets were lost over the measurement interval. To filter the fluctuations, the PER is calculated by a moving average over a PER window interval, $(w)$, which is divided into sub-windows, $(s w)$. Thus, $=\eta \times s w$, where $\eta$ is an integer number greater than one $(\eta>1)$. The number of missed packets is 
calculated at the end of each sub-window interval. Then, PER is calculated for the last $\eta$ subwindows as follows

$$
P E R_{i}(j)=\frac{\# \text { of missed packets from } j \text { in }\left[s w_{c u r-\eta}, s w_{c u r}\right]}{\text { Total \# of packets from } j \text { in }\left[s w_{c u r-\eta}, s w_{c u r}\right]}
$$

Therefore, the success probability of Bernoulli trial of embedding the TDU forwarded by vehicle $j$ in next beacon of vehicle $i$ is calculated as

$$
p_{i}(j)=\min \left(\frac{\operatorname{ISPF}(d(i, j))}{1-P E R_{i}(j)}, 1\right)
$$

where $\operatorname{ISPF}(d(i, j))$ is the ideal success probability function of rebroadcast for vehicle $i$. If the channel condition was not an issue and no packet would get lost, the success probability would have been equal to the output value of ISPF and could have been calculated only based on the distance of two vehicles as the denominator would always be one. However, in reality due to increase of attenuation and collision, PER increases by distance. Therefore, to compensate for the higher PER which would lower the chance of transmission, the probability of transmission is strengthened. The minimum function assures that the success probability never exceeds one. It can be noted that the Bernoulli trials are not identical due to the success probability of (3.7), though they are still independent. In probability theory this distribution is called Poisson binomial distribution with $N$ trials.

One possible choice of ISPF could have been the Bernoulli trials with identical success probability, like the previous example of uniform probabilistic method. However, closer vehicles would have got higher chance of forwarding since the numerator of the fraction in (3.7) would be the same for all vehicles but the denominator would have got smaller as PER increases by distance.

While the behavior of PER completely depends on the network conditions, our protocol defines the ISPF in a way to compensate the effect of PER to a great extent. As it was mentioned before, it is ideal to let the farthest vehicle in a single-hop communication range to forward the message. Moreover, adequate increase of the numerator of the fraction in (3.7) by distance, can eliminate the negative effect of PER and give farther vehicles higher probability. In our approach, we propose an $I S P F$, which is defined as

$$
\operatorname{ISPF}(d(i, j))=\frac{\left(1-\exp \left(\lambda_{i} \times \frac{d(i, j)}{D\left(P_{t x}\right)}\right)\right)}{1-\exp \left(\lambda_{i}\right)}, \quad \lambda_{i}>0
$$

where $\lambda_{i}$ is the scale parameter that changes adaptively based on the density and average speed of the vehicles in the single-hop communication range of vehicle $i$. It can be noted that since for all $\lambda_{i}>0,\left(1-\exp \left(\lambda_{i}\right)\right)<0$, so the function is monotonically increasing by distance.

As it is shown in [139], distribution of vehicles in a fully connected unidimensional network has an exponential distribution. Consequently, the tail probability of inter-vehicle distance is also exponential, which means the chance of a vehicle existing in farther distances is exponentially 
decreasing. As a result, the function in (3.8) is chosen as a normalized exponentially increasing function to compensate the lower probability of vehicle existence in farther distances, with a higher chance of retransmission. In the free-flow traffic, the value of $\lambda_{i}$ represents the traffic flow (in vehicles per second), while the inter-arrival time is an exponential distribution with parameter $\lambda_{i}$ and the average speed is independent from the traffic flow and density. Therefore, it can be calculated as $\lambda_{i}=\bar{v} \times \rho$, where $\bar{v}$ is the average speed of in-range vehicles [139]. However, in a congested and force-flow traffic state, where the average speed starts to decrease due to formation of queues of vehicles and becomes related to the value of density, the above formula is not valid and cannot be used. Because the value of $\lambda_{i}$ affects the success probability, in such traffic states the value of $\lambda_{i}$ should be adapted based on the channel load condition and required forwarder ratio which is described in the next section.

\subsubsection{Analysis of Algorithm}

It is important to determine the amount of forwarder ratio and channel load as it should be limited and comparable. Since piggybacking over beacons is used, there is no independent message forwarding and so no explicit forwarder exists. Hence, the summation of the average number of TDUs per beacon, divided by the number of in-range vehicles implicitly expresses the forwarder ratio. Consequently, channel load means the amount of extra load added to the channel due to attaching of TDUs to BSM packets. Therefore, the average amount of channel load for each vehicle (in bits per second) is determined by the product of average number of TDUs included inside a BSM per beacon, and the size of each TDU $\left(\ell_{\text {TDU }}\right)$. This value shows the amount of communication overhead which is added to the network by our proposed protocol. Considering the fact that the size of TDU is application dependent, fixed, and equal for all vehicles, calculation of the expected value of the number of TDUs per vehicle per beacon determines both forwarder ratio and channel load.

Let $X_{j}^{i}(r)$ denote the number of TDUs forwarded by vehicle $j$ in its $r$-th beacon, so $\mathbb{E}_{r}\left[X_{j}^{i}(r)\right]$ shows the expected value of number of TDUs per beacon for vehicle $j$. Note that vehicle $j$ is an arbitrary vehicle in the communication range of vehicle $i$. Therefore, $\left(\ell_{\mathrm{TDU}} \times \mathbb{E}_{r}\left[X_{j}^{i}(r)\right]\right)$ shows the average amount of added load for vehicle $j$. Averaging over all vehicles in the communication range of vehicle $i$, represents the forwarder ratio in that range, which is shown as $\mathbb{E}_{j}\left[\mathbb{E}_{r}\left[X_{j}^{i}(r)\right]\right]$ $\forall j \neq i$.

For the purpose of comparison, when each vehicle creates its own TDU, this ratio for the probabilistic approach with identical Bernoulli trials is equal to

$$
\mathbb{E}_{j}\left[\mathbb{E}_{r}\left[X_{j}^{i}(r)\right]\right]_{\text {uniform prob. }}=\frac{N \times\left[N \times \frac{\delta}{N}\right]}{N}=\delta
$$

The higher $\delta$ results in more rebroadcast of the same TDU, which means more reliability but higher channel load, and that is the reason $\delta$ has been called as the reliability factor.

The expected value of number of TDUs per vehicle per beacon in our approach can be found as 
$\mathbb{E}_{j}\left[\mathbb{E}_{r}\left[X_{j}^{i}(r)\right]\right]_{N 2 D C A S T}=\frac{\sum_{j=1}^{N} N \times I S P F(d(i, j))}{N}=\sum_{j=1}^{N} \operatorname{ISPF}(d(i, j)), \forall j \in\{$ in range of $i\}$

where $N$ vehicles exist in a single-hop communication range of $D\left(P_{t x}\right)$. Dividing the communication range into $N$ bins, $D\left(P_{t x}\right) / N$, shows the average space between vehicles. Therefore, the distance of vehicle $i$ from vehicle $j$, which is located at the $k$-th bin, can be approximated by $d(i, j) \cong k \times\left(\frac{D\left(P_{t x}\right)}{N}\right)$. In consequence, the fraction $\left(\frac{d(i, j)}{D\left(P_{t x}\right)}\right)$ can be discretized as $\left(\frac{k}{N}\right)$ and by replacing $\theta_{i}=1-e^{\lambda_{i}}$ in Equation (3.8), it can be rewritten as

$$
\operatorname{ISPF}(d(i, j)) \cong \operatorname{ISPF}(k)=\frac{1-\left(1-\theta_{i}\right)^{\left(\frac{k}{N}\right)}}{\theta_{i}}
$$

Therefore, the expected value can be calculated as

$$
\mathbb{E}_{j}\left[\mathbb{E}_{r}\left[X_{j}^{i}(r)\right]\right]_{N 2 D C A S T} \cong \sum_{k=1}^{N} \operatorname{ISPF}(k)=1+\frac{N}{\theta_{i}}+\frac{1}{\left(1-\theta_{i}\right)^{\frac{1}{N}}-1}
$$

The left side of Equation (3.12) is equal to the reliability factor of the uniform probabilistic approach if the same forwarder ratio is desired. Therefore, in consideration of any preferred reliability factor, $(1 \leq \delta \leq N)$, the value of $\theta_{i}$ can be derived from $\delta=1+\frac{N}{\theta_{i}}+\frac{1}{\left(1-\theta_{i}\right)^{\frac{1}{N}-1}}$. Since this expression does not have a parametric closed form solution for $\theta_{i}$, the value of $\lambda_{i}=\ln \left(1-\theta_{i}\right)$ is numerically approximated before each beacon using the Padé approximation, with respect to the current value of $N$ for vehicle $i$, and the preset value of $\delta$.

\subsection{Formal protocol specification}

A formal specification of the proposed protocol is presented in pseudo-code in Figure 3-2 which shows the program that runs at each vehicle in the form of $<$ event $\rightarrow$ action $>$ pairs. 


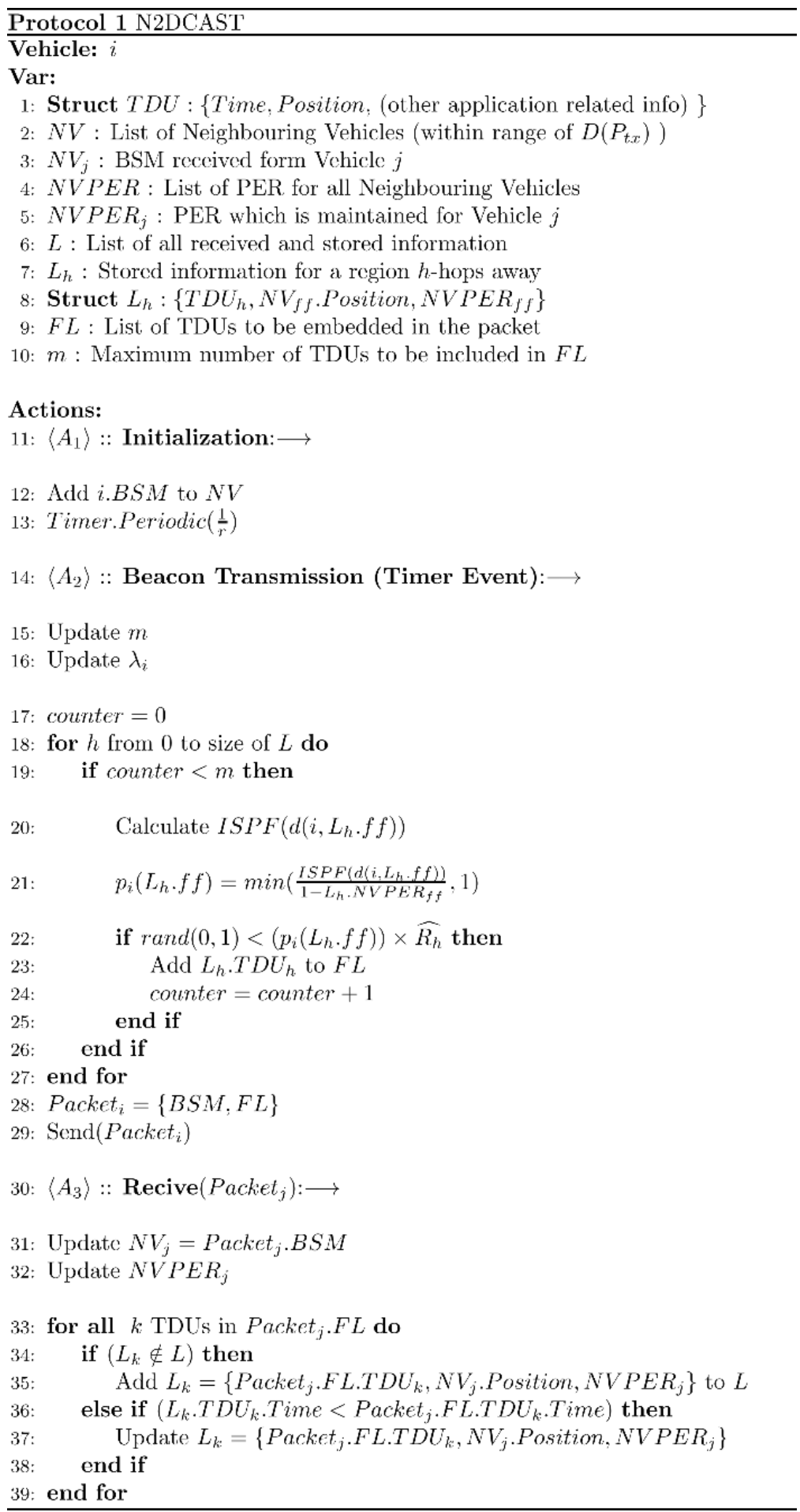

Figure 3-2- Description of N2DCAST protocol.

In the proposed method, each vehicle keeps track of all in-range neighboring vehicles through their beacons in a list which is shown by $N V$. New in-range vehicles are added to the list upon the reception of their first beacon. Moreover, based on a pre-defined time interval, if no packets were 
received from a specific vehicle in the list, the list is updated to remove that vehicle, as it is considered not in the communication range anymore. Therefore, length of $N V$ shows the number of vehicles in-range, which is earlier denoted by $N$. Furthermore, each vehicle carries a list of all TDUs which have been received up to now, and is shown by $L$. An old TDU is replaced at reception of a newer one for the same region. The corresponding TDU for each hop along with other related parameters are stored inside each item of $L$. For example, $L_{0}$ contains the TDU of the communication range of vehicle $i$, and $L_{h}$ has the latest received information for $h$-hops away from it. The extra stored parameters are the position of the first in-range forwarder $(f f)$ which sent the TDU and the PER which has been maintained for it.

Each vehicle transmits its single-hop BSM beacons every $\frac{1}{r}$ seconds. The maximum number of allowed TDUs to be added $(m)$ and the success probability of inclusion of each TDU based on current density is updated before each transmission. Subsequently, each vehicle creates a forwarding list (i.e. $F L$ ), based on the value of $m$ and the previously explained probabilistic distance-dependent approach. TDUs will be added to $F L$ of next beacon if they satisfy all the protocol conditions.

Upon reception of a packet, each vehicle separates the BSM from the TDUs, and then each TDU is used to update the corresponding record in $L$. If the vehicle has never received any TDU for this hop, a new record will be added to the list, and if not, the existing record will be updated with the recently received TDU only when it has a posterior timestamp.

\subsection{Performance Evaluation}

To evaluate the performance of our algorithm, we use ns-3, which is a powerful network simulator platform and has a comprehensive implementation of $\mathrm{V} 2 \mathrm{~V}$ communication. In our ns-3 simulations, DSRC specifications are configured for media access control (MAC) and physical (PHY) layers. The MAC layer protocol is CSMA/CA with enhanced distributed channel access (EDCA) at $5.9 \mathrm{GHz}$ frequency band, and the PHY layer is configured based on $802.11 \mathrm{p}$ protocol with $10 \mathrm{MHz}$ bandwidth and OFDM bit rate of $6 \mathrm{Mbps}$. Other constant parameters are summarized in Table 3-1. In order to focus on the performance of the proposed method, the transmission rate and range are fixed at $r=10 \mathrm{~Hz}$ and $D\left(P_{t x}\right)=500 \mathrm{~m}$, respectively. 
Table 3-1- ns-3 Simulation Parameters

\begin{tabular}{|c|c|c|c|}
\hline Parameter & Value & Parameter & Value \\
\hline Noise Floor & $-99 \mathrm{dBm}$ & $\overline{\text { AIFSN }}$ & 7 \\
\hline $\begin{array}{l}\text { Carrier-sense } \\
\text { Threshold }\end{array}$ & $-94 \mathrm{dBm}$ & $\begin{array}{l}\text { Contention } \\
\text { Window }\end{array}$ & 15 \\
\hline $\begin{array}{l}\text { Packet } \\
\text { Reception } \\
\text { SINR }\end{array}$ & $7 \mathrm{dBm}$ & $\begin{array}{l}\text { Channel } \\
\text { Bandwidth }\end{array}$ & $10 \mathrm{MHz}$ \\
\hline $\begin{array}{l}\text { Transmission } \\
\text { Rate }\end{array}$ & $\begin{array}{r}10 \mathrm{~Hz} \\
\text { (Fixed) }\end{array}$ & $\begin{array}{l}\text { Inter-hop } \\
\text { Forwarding } \\
\text { Rate }\left(\widehat{R_{h}}\right)\end{array}$ & $\frac{1}{h+1}$ \\
\hline $\begin{array}{l}\text { Channel } \\
\text { Capacity } \\
\text { Utilization } \\
\text { Ratio }(\beta)\end{array}$ & 0.05 & $\begin{array}{l}\text { Simulation } \\
\text { Time }\end{array}$ & $100 \mathrm{sec}$ \\
\hline BSM Size & 306 Bytes & TDU Size & 52 Bytes \\
\hline $\begin{array}{l}\text { Packetization } \\
\text { Overhead } \\
\text { Ratio }(\gamma) \\
\end{array}$ & 0.1 & $\begin{array}{l}\text { OFDM Bit } \\
\text { Rate }\end{array}$ & $6 \mathrm{Mbps}$ \\
\hline Range & $\begin{array}{r}500 \\
\text { meters }\end{array}$ & $\begin{array}{l}\text { Transmissi } \\
\text { on Power }\end{array}$ & $10 \mathrm{dBm}$ \\
\hline $\begin{array}{l}\text { PER window } \\
(w)\end{array}$ & $5 \mathrm{sec}$ & $\begin{array}{l}\text { PER sub- } \\
\text { window } \\
(s w)\end{array}$ & $1 \mathrm{sec}$ \\
\hline
\end{tabular}

Since communication channel affects the packet reception due to attenuation and packet collision, to study the performance of the algorithm in terms of the communication channel (i.e. PER), two different channel propagation models are used. The first model is Fix-Range propagation, which is an ideal channel with no attenuation up to a predetermined range (500 meters) and 100\% attenuation and loss after that. The second channel model is Three-Log propagation with Nakagami fading (ns-3 default values are used for the parameters of this model). The transmission power is set to $10 \mathrm{dBm}$, to have an equivalent transmission range of 500 meters (defined as the distance at which at least $10 \%$ of packets are successfully received).

Two topological scenarios are considered to study the effect of vehicle movements. In the first scenario, vehicles are moving with the constant speed of 20 meters per second and uniformly distributed on the first 7 kilometers of a 10-kilometer highway with 4 lanes. Moreover, two different vehicle densities of 0.04 and 0.2 vehicles per meter are used to analyze the effect of message size control scheme. The second scenario is created by the simulator of urban mobility (SUMO) on the same highway, where the speed of vehicles varies from 19 to 25 meters per second.

Finally, as there is no comparable method in the literature, modified versions of the uniform probabilistic method and counter-based method are used.

The averaged recoded PER in each vehicle versus distance, for the entire simulation time is shown in Figure 3-3 and Figure 3-4 for two different channel propagation models, Fix-Range propagation, and Three-Log propagation, respectively. PER curves for the counter-based rebroadcast method with the size control scheme are identical to the curves of our method, thus we plotted only one of 
them. However, to illustrate the effect of message size control on PER, the curves for a counterbased method with no message size control are plotted.

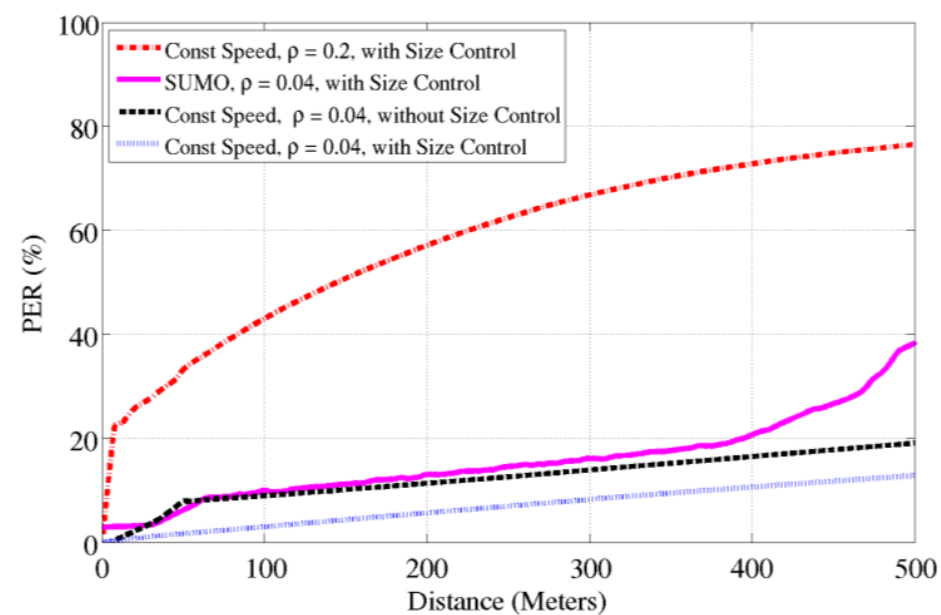

Figure 3-3- PER versus distance for constant speed and SUMO scenarios with two different vehicle densities for Fix-Range channel propagation model. All scenarios, except the one noted, have message size control.

As it is shown in Figure 3-3, although the communication channel is ideal, still some packets were dropped due to the hidden-node phenomenon [106].

Hidden-node happens when two vehicles, which are not in the communication range of each other, transmit simultaneously to a common destination since the channel was sensed idle in both of them. In this case, a collision happens and both packets get dropped at the intended destination. Since communication range of farther vehicles has less overlap, the chance of collision due to hidden-node is higher for them. Furthermore, higher vehicle density means more hidden node collisions because more vehicles try to utilize the channel.

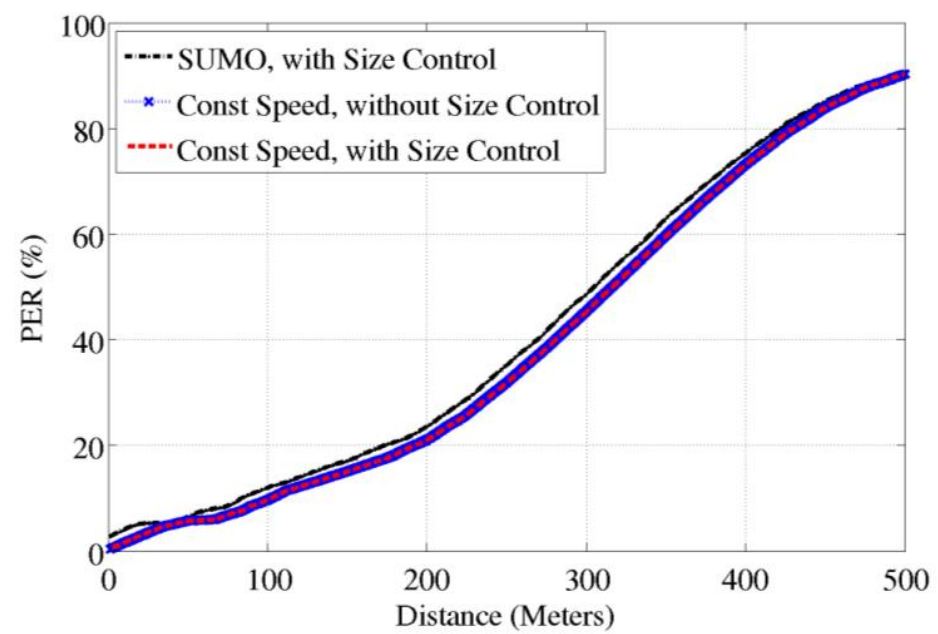

Figure 3-4- PER versus distance for constant speed and SUMO scenarios with Three-Log channel propagation model and density of $\rho=0.04$. 
The success probability of Bernoulli trials versus distance $\left(p_{i}(j)\right)$ is shown in Figure 3-5. Considering the PER curve for each scenario from above figures, each vehicle uses its own ISPF to calculate the probability of rebroadcast for each TDU. The curve of ISPF (Equation (3.8)) versus distance with parameter $\lambda_{i}=3.8$ is also shown in Figure 3-5. This value is approximated with respect to the reliability factor of $\delta=10$ and vehicle density of $\rho=0.04$ vehicles per meter. The success probability of uniform probabilistic method is also shown with the same value of reliability factor and density. As this figure shows, the success probability of rebroadcast does not only depend on ISPF and distance, but also PER and channel conditions. Therefore, the farthest vehicle would not necessarily be the next forwarder as other vehicles may obtain higher probability based on their conditions.

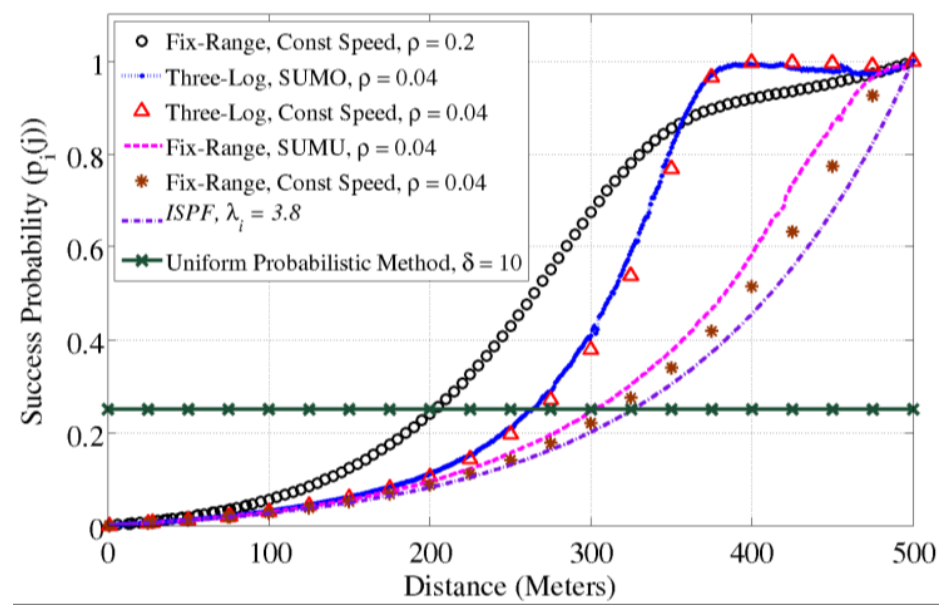

Figure 3-5- Success probability of rebroadcast versus distance for constant speed and SUMO scenarios, two vehicle densities, and two propagation models. The curve of $I S P F$ (Equation (3.8)) versus distance with parameter $\lambda_{i}=3.8$ and uniform probabilistic method with parameter $\delta=10$ are also shown.

Figure 3-6 shows the average number of TDUs per beacon for each vehicle, in probabilistic, counter-based, and N2DCAST rebroadcast methods when all of them use the message size control scheme. This value is equal to $\mathbb{E}_{r}\left[X_{j}^{i}(r)\right]$, which is the expected value of number of TDUs per beacon for vehicle $j$. Therefore, the amount of added channel load for each vehicle can be determined where TDU size is 52 Bytes. The results are only shown for constant speed scenarios since the relative distance of the vehicles is fixed during the simulation runtime. While the scattered values for counter-based method show a random behavior along the road, the probabilistic method and N2DCAST change their behaviors based on the network density measured by each vehicle. The density is lower on the edges of the road so there is less congestion in that area and vehicles get a higher chance to transmit. The forwarder ratio of the entire road is also determined by averaging over all vehicles. As this figure shows, having the same road density and reliability factor, the forwarder ratio and channel load of all three methods are close to each other. 


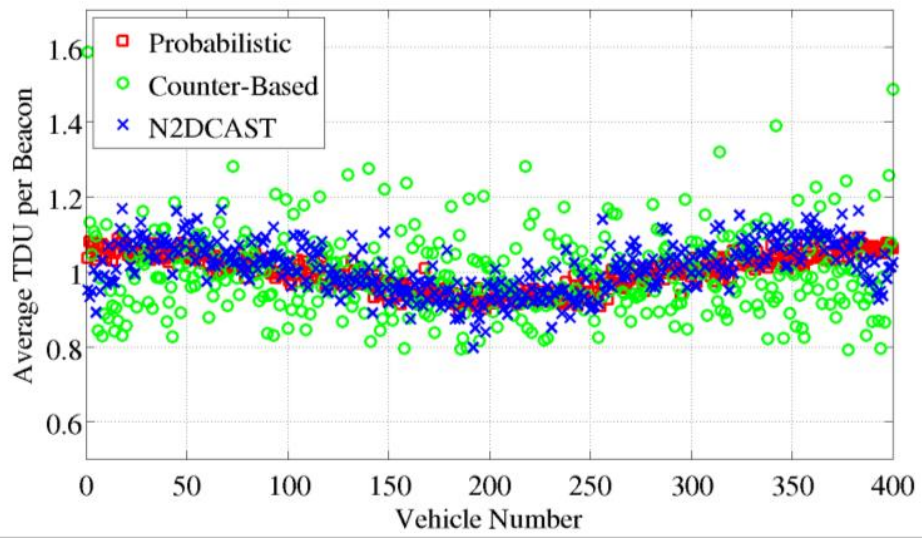

Figure 3-6- Average number of TDUs per beacon for each vehicle along the road for different rebroadcast methods. The forwarder ratio, which is averaged over all vehicles, is $0.9984,0.9958$, and 1.012 for probabilistic, counter-based, and N2DCAST methods, respectively.

Figure 3-7 depicts the staleness results for Fix-Range channel propagation. To make the comparison fair and legitimate, the message size control algorithm and the second layer of our distance-dependent (inter-hop) scheme exist in all of these different methods. The results not only show that our approach outperforms other methods, but also they show that the performance of our method is not affected by vehicle density. Moreover, since the chance of existing vehicles at farther distances is more, the performance is slightly better. Aside from that, these results also imply that by choosing the right value of $\delta$, uniform probabilistic method and counter-based method are equivalent. Furthermore, the significant effect of message size control scheme on staleness is also noticeable in this figure.

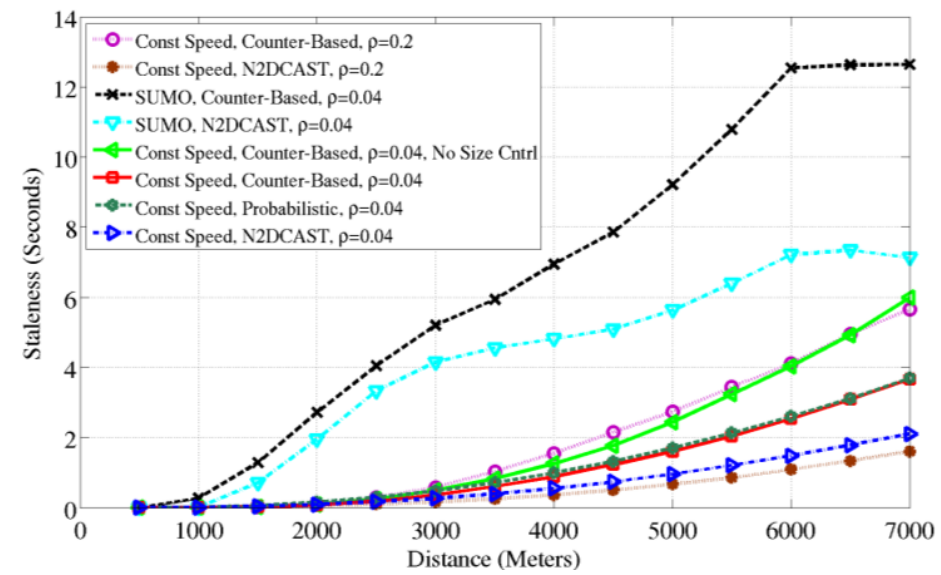

Figure 3-7- Staleness for each hop versus distance for constant speed and SUMO scenarios, two vehicle densities, and Fix-Range propagation model.

All scenarios, except the one noted, have message size control.

The staleness results for Three-Log channel propagation model are shown in Figure 3-8. Each point represents the average value of staleness in all vehicles at that hop. 


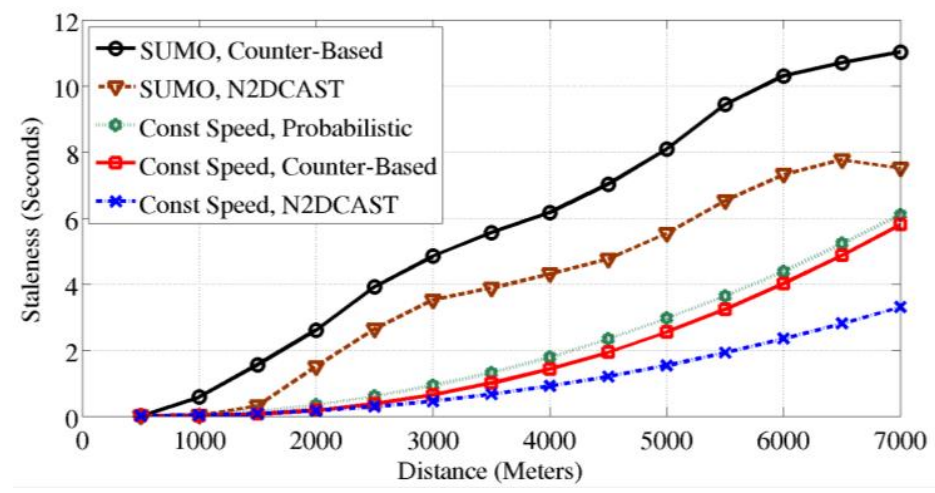

Figure 3-8- Staleness versus distance for constant speed and SUMO scenarios with Three-Log propagation and vehicle density of $\rho=0.04$. 


\section{Chapter 4 Situational Awareness and Hazard Detection for Vulnerable Road Users}

The National Highway Traffic Safety Administration (NHTSA) estimated around five million crashes annually and more than thirty thousand fatalities in the United States in 2011. Despite the noticeable efforts of vehicle manufacturers, Vulnerable Road Users (VRUs) such as pedestrians, people with disabilities, cyclists, motorcyclists, public safety personnel, and road workers still account for a significant portion of accident fatalities [70]. Driver distraction and obstruction invisibility, or VRU unawareness of the severity of imminent danger are among the primary reasons behind these fatalities. Although the number of crash victims has been reduced by almost fifty percent since the 1970s, when safety features in vehicles were gradually improved [87, 140], according to NHTSA, in 2011 traffic crashes caused more than four thousand pedestrian fatalities and resulted in more than seventy-five thousand pedestrian injuries in the United States alone [141]. Moreover, the reports show that around seven hundred cyclists lost their lives and about fifty thousand were injured in traffic crashes. In addition, more than one hundred road workers were killed in the United States during 2011.

On the other hand, preparatory studies show that more than eighty percent of unimpaired traffic accidents are expected to be prevented by safety applications based on V2V communications [87]. The development of V2V safety applications and their standardization has been ongoing for more than a decade, and they are anticipated to be deployed within three years. However, such safety applications are seriously missing for VRUs.

In this chapter, we propose, design, and implement an entire V2P framework which complements and improves our previous prototype in [89]. First, existing literature on VRU safety systems is summarized. We afterward discuss the most common and deadliest crash scenarios, to justify our design approach. Subsequently, the proposed V2P framework is described. The VRU safety module is then explained in detail, including target classification and collision detection algorithms. Furthermore, we propose and evaluate a mitigating solution for congestion and power consumption issues in these systems. The results of our outdoor test scenario for the implemented framework are then presented, for which a Qualcomm DSRC-enabled smartphone and a Hyundai- 
Kia DSRC-equipped vehicle are used (Figure 4-1). Finally, we provide our conclusions and future plans.

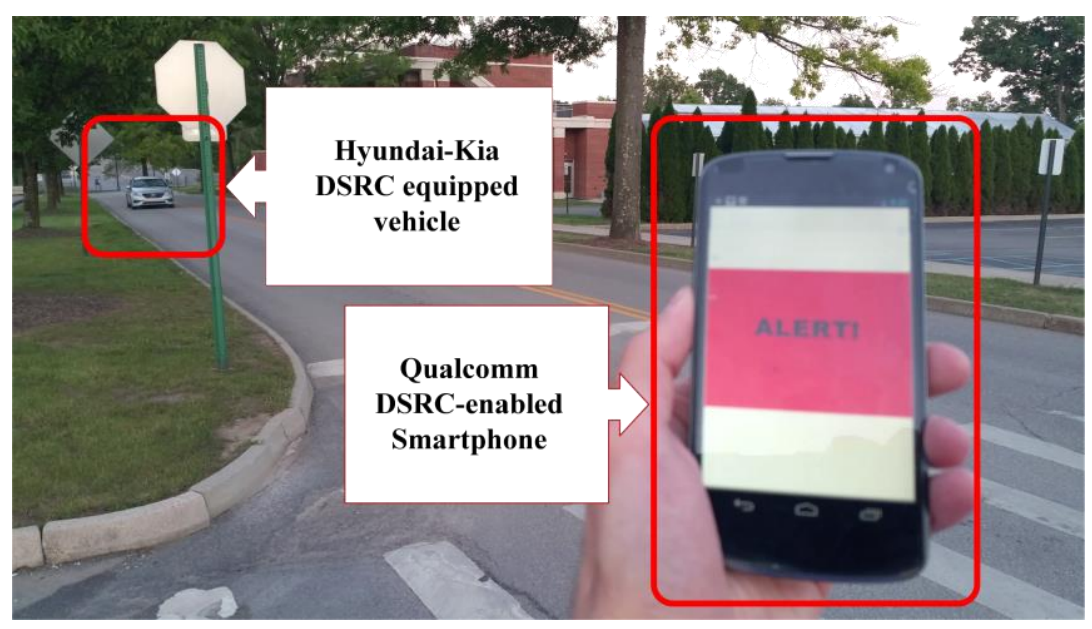

Figure 4-1- Our cooperative VRU safety system

\subsection{Pedestrian Crash Scenarios}

While vehicle to VRU accidents could happen almost anywhere, NHTSA reports the following as the most common and injury-prone crash scenarios [82]:

1) Pedestrian crossing the road in front of straight going vehicle (Figure 4-2-a).

2) Pedestrian crossing the road in front of right-turning vehicle at the intersection (Figure 4-2b).

3) Pedestrian crossing the road in front of left-turning vehicle at the intersection (Figure 4-2c).

4) Pedestrian walking beside the road and vehicle going straight (Figure 4-2-d). 


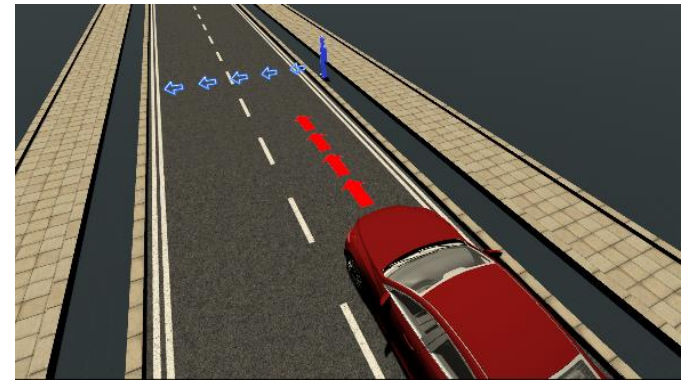

a) Pedestrian crossing

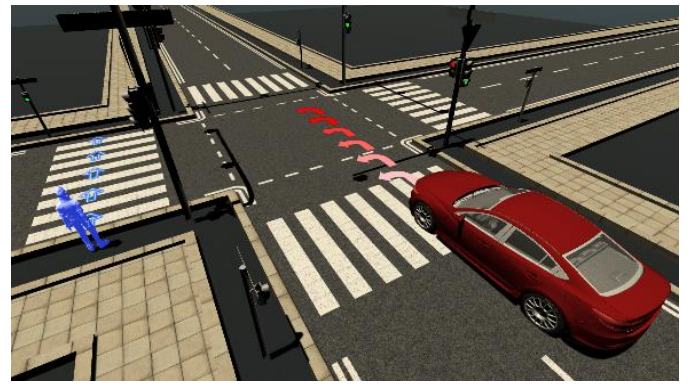

c) Intersection Left Turn

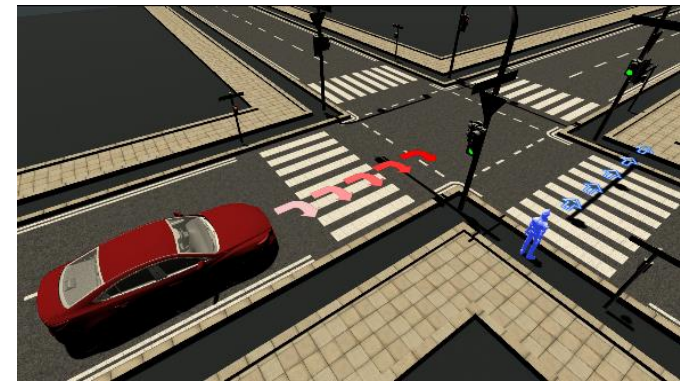

b) Intersection Right Turn

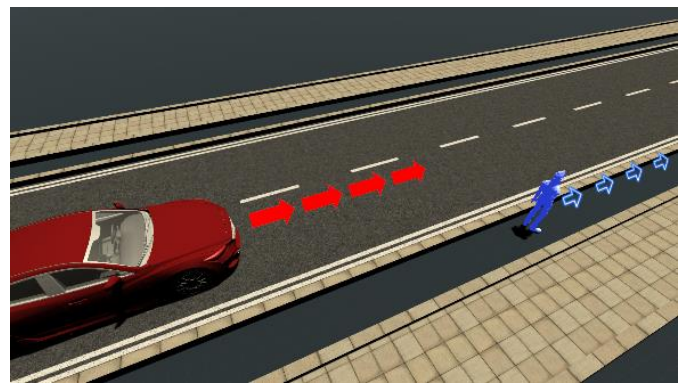

d) Pedestrian beside road

Figure 4-2- the most common and injury-prone vehicle-pedestrian crash scenarios

Although these four scenarios account for only $46 \%$ of all crashes, they are reported for $98 \%$ of fatalities, injuries, and monetary damages of all pedestrian crashes. Therefore, the focus of our VRU cooperative safety module stems from these four scenarios. Based on the NHTSA report, $78 \%$ of accidents happen when pedestrians are crossing improperly, running onto the road, or being distracted. On the vehicle side, $87 \%$ of accidents happen when vehicles are going straight or turning left or right [70].

The most common crash scenario is the first one in which a crossing pedestrian is hit by a straight going vehicle. The second and third scenarios are typical turning accidents at intersections. Although turning accidents consist of the complicated vehicle and pedestrian maneuvers, the injuries are generally less intense. Finally, the fourth scenario, which is a fatal case, involves fast and unforeseeable maneuvers in which pedestrians are walking along the street without even intersecting the path of vehicles.

\subsection{Proposed V2P framework}

As aforementioned, obstructed VRUs cannot be timely detected by sensors such as radars and cameras. Therefore, communication-based cooperative methods with omnidirectional and extended situational awareness range should be employed to address such hazardous scenarios.

Our proposed V2P framework is shown in Figure 4-3, in which the vehicle and VRU are assumed to be equipped with a wireless safety unit (WSU) and a DSRC-enabled smartphone, respectively. The WSU is connected to the controller area network bus (CAN bus) and a GPS receiver. The CAN bus continuously provides the WSU with all sensory data collected by local sensors, such as the odometer, accelerometer, brake status, turn signals, and radar, which express the current state 
of the vehicle. The GPS receiver periodically feeds the location information into the WSU and might also support offline Geographical Information System (GIS) maps, like Google maps, which can help identifying static structures and road geometries. Moreover, both the WSU and the smartphone contain a DSRC communication module, which is capable of communicating SAE J2735 messages, such as basic safety messages (BSMs) and personal safety messages (PSMs). Subsequently, all of this information is fed into a situational awareness subsystem to create the extended real-time map of the surrounding environment.

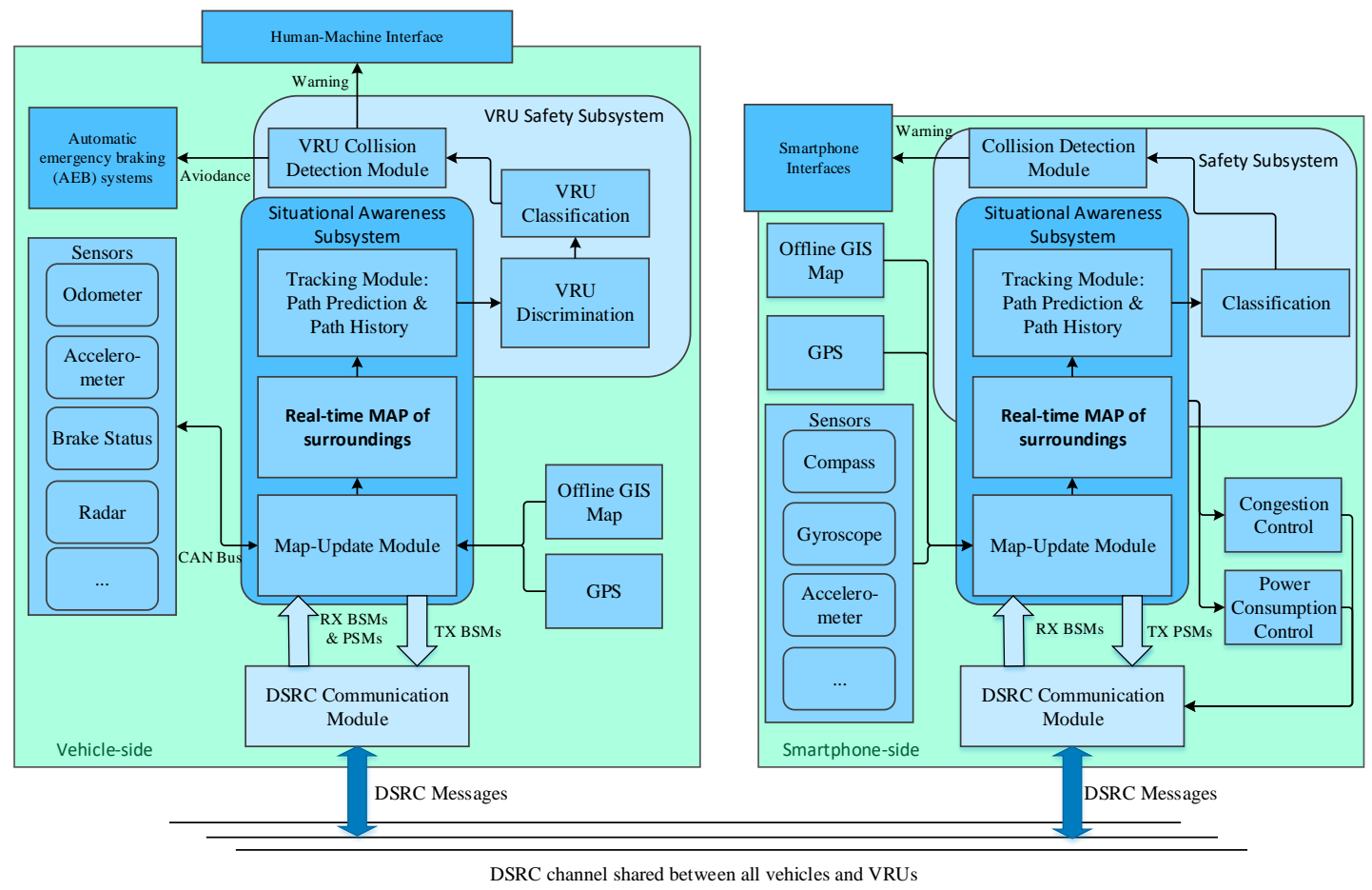

Figure 4-3- Proposed cooperative VRU safety framework

The situational awareness subsystem consists of three components: map-update module, surroundings real-time map, and tracking module. The map-update module is in charge of refreshing the real-time map with the latest received information from sensors, GPS, and DSRC communication module. Thus, the real-time map database keeps records of its surrounding environment, such as all neighboring vehicles, VRUs, and other detected objects. Every map record consists of the latest available information about a particular neighboring entity, such as its position information, speed, the size of the vehicle, or type of VRU. The map is created and updated based on the latest self-position as the origin and the latest heading direction as the $\mathrm{x}$-axis of its coordinate system. In order to formulate this coordinate system, the GPS information, which is in the World Geodetic System 1984 format (WGS 84), is converted into the Earth-Centered, Earth-Fixed format (ECEF). The ECEF format is then transformed into East-North-Up (ENU) coordinates. The ENU coordinates are finally rotated toward the latest heading direction. Additionally, the tracking module utilizes the previous and current map records to construct the path history points and path prediction points for all map elements. This information is then used by safety subsystems to identify pre-crash scenarios. Furthermore, the situational awareness 
subsystem is responsible for delivering the latest information to the communication module to be broadcast to other vehicles and VRUs.

On the vehicle-side, the VRU safety subsystem utilizes the results of the situational awareness subsystem to determine possible hazardous situations and generate appropriate notifications and well-timed signals. This subsystem consists of VRU discrimination component, VRU classification component, and VRU collision detection module. The VRU discrimination component distinguishes between different types of VRUs, as specific VRUs may require different classification or collision detection approaches. This requirement is also considered in PSM that has a field for device user type, which describes the type of non-vehicular road user whose condition information is being transmitted. These types have been exemplified in SAE J2735 as pedestrians, pedal cyclists, public safety workers, and animals. Nevertheless, this information might be unavailable explicitly, especially when the device is a smartphone since they can be carried by pedestrians, cyclists, road workers, and even vehicle passengers. Therefore, whenever the field is blank, both the smartphone and the counterpart vehicle should detect and identify the VRU type to the greatest extent.

Although numerous PSM fields could be helpful in various situations, the most informative candidates are velocity, four-way acceleration set, path history, and path prediction. Velocity could help to differentiate between pedestrians, pedal cyclists, and motorcyclists. It can also speculate the age, disability, and impairment of pedestrians. Four-way acceleration set, which is the set of lateral, longitudinal, and vertical accelerations along with yaw rate could also be used to discriminate between pedestrians and motorized road users. Path history and path prediction of a VRU could determine whether the VRU is crossing the road or staying within the boundaries of the road, which not only can separate riders from pedestrians but also public safety and road workers from other pedestrians. After the VRU type has been determined, the smartphone can include it in the PSM, and the vehicle VRU discrimination component can feed it into the VRU classification component.

The VRU classification component executes a target classification algorithm to label safe, risk, or danger zones based on current vehicle dynamics (Figure 4-4). This information is then used by the collision detection module to generate prompt warnings or activate collision avoidance actuators. The warnings are then interpreted by the human-machine interface (HMI) and could be in the form of visual, auditory, or haptic alarms. The collision avoidance signals trigger evasive mechanisms such as automatic emergency braking (AEB) or crash imminent braking (CIB). The details of our proposed algorithm for VRU classification and collision detection module is provided in next subsection. 


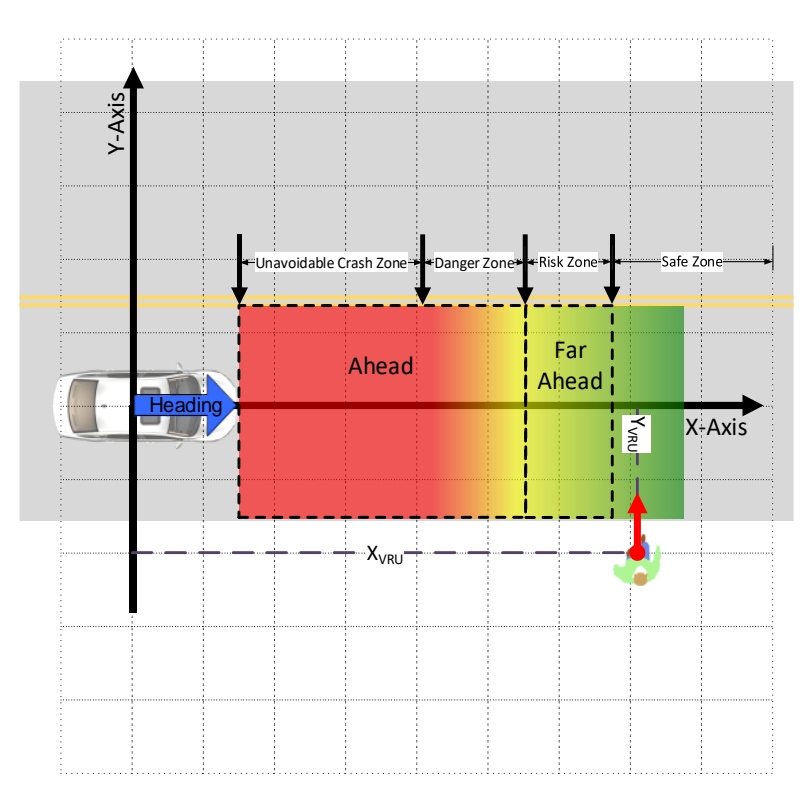

a) Straight path

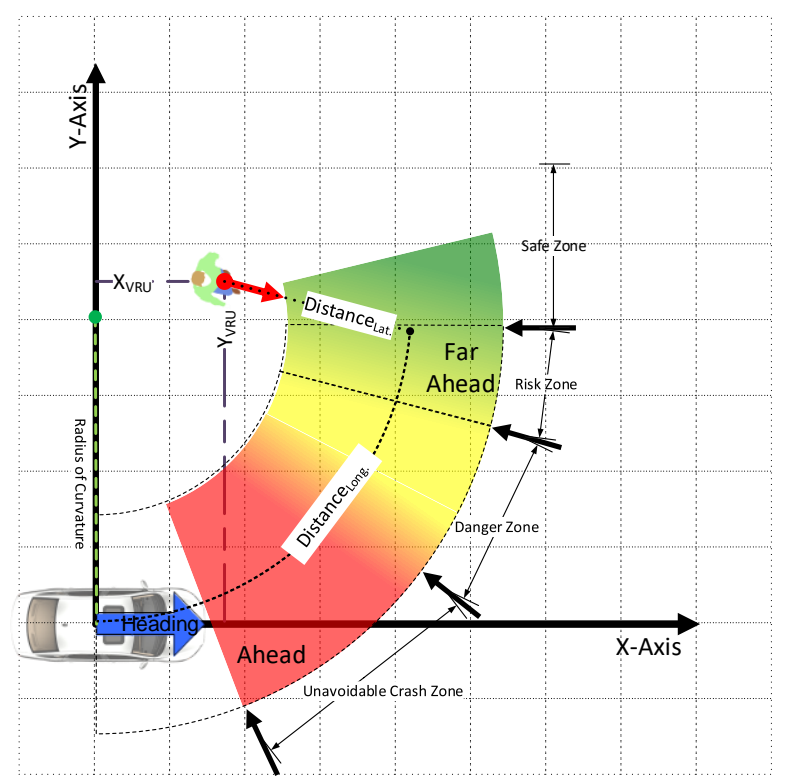

b) Curved path

Figure 4-4- Different severities of possible crashes in straight and curved paths

The safety subsystem of the smartphone is designed conceptually similar to VRU safety subsystem of the vehicle, except that it does not require the discrimination component, has different HMI capabilities, and runs a slightly modified detection algorithm. In addition, smartphone-side contains two extra modules, namely congestion control and power consumption control. The congestion control module mitigates the communication load of DSRC network in crowded and dense areas, and the power consumption control module optimizes battery usage by minimizing the overall safety system duty cycle.

\subsubsection{Target Classification and Collision Detection Algorithms}

We design the target classification and collision detection algorithms to separate and alert the vehicles or VRUs based on their current movement patterns if they might encounter an accident. The classification, detection, and alerting should be prompt enough to prevent crashes while counting for the driver reaction delay. However, too early alarms may be false positive and breed mistrust toward the whole system.

Taking into consideration the kinematic constraints of vehicles and hazardous areas around them, we define different zones based on the severity of possible crashes in our target classification algorithm. Figure 4-4 illustrates these zones conceptually, in which the width of each zone is equal to lane width, and the length of it at each moment is dependent on the speed and acceleration of the vehicle. The target classification algorithm has the responsibility of assigning the corresponding zone labels and updating them periodically.

The unavoidable crash zone is the zone inside which the accident is inevitable, even with the most immediate harshest possible brake. The minimum time-to-stop $\left(\right.$ TT $\left._{\min }\right)$ is defined as the time it 
takes to stop the vehicle with maximum braking deceleration $\left(d_{\max }\right)$, based on current speed $\left(v_{c u r}\right)$, current acceleration $\left(a_{c u r}\right)$, and driver reaction delay $\left(T_{D R D}\right)$.

$$
T T S_{\text {min }}=-\frac{v_{b r k}}{d_{\max }}+T_{D R D}
$$

where $v_{b r k}$ is the speed of vehicle after driver reaction delay and equals to $v_{b r k}=a_{c u r} \times T_{D R D}+$ $v_{c u r}$. The corresponding travelled distance, which is defined as the minimum distance-to-stop $\left(D T S_{\min }\right)$, determines the length of unavoidable crash zone an can be calculated as

$$
D T S_{\min }=D_{D R D}+\left(-\frac{v_{b r k}^{2}}{2 \times d_{\max }}\right)
$$

where $D_{D R D}$ is the traveled distance during driver reaction delay

$$
D_{D R D}=\frac{1}{2} \times a_{c u r} \times T_{D R D}^{2}+v_{c u r} \times T_{D R D}
$$

Coming after is the danger zone, in which a certain forthcoming accident is avoidable if a prompt warning is generated for the driver. Such warning is known as an imminent warning and could be followed up by AEB or CIB signal to prevent the crash. The length of danger zone is specified by the distance traveled during $T_{i w}=T T S_{\min }+T_{\text {guard }}$, which is denoted by the guard distance-tostop $\left(D T S_{\text {guard }}\right)$. $T_{\text {guard }}$ determines how soon the first imminent warning should be generated after a crash is detected, assuming that the driver reaction delay is already considered in $T T S_{\min }$. Therefore, the level of conservatism of method can be adjusted by $T_{\text {guard }}$, considering that there is always a trade-off between the level of conservatism and too early disruptive false alarms.

In spite of this trade-off, the driver should still be informed and advised about a probable accident to react moderately with peace of mind, which results in a smoother braking behavior and more comfortable ride. Accordingly, the region for which the driver should only be advised about the possibility of an accident by an advisory warning is called the risk zone. Therefore, the length of the risk zone is dependent on the time it takes for the vehicle to stop with a moderate braking deceleration $\left(d_{\text {mod }}\right)$, considering driver reaction delay and current vehicle velocity and acceleration. This duration is denoted by $T_{a w}=T_{i w}+T_{\text {mod }}$, in which $T_{\text {mod }}$ is added for the sake of gentle braking. The corresponding travelled distance is defined as the moderate distance-to-stop $\left(D T S_{\text {mod }}\right)$, which explains the length of risk zone. The regions beyond these zones are considered as safe.

The dimensions of each zone should be recalculated frequently. Each VRU is afterward classified in either safe, risk, or danger zone based on its estimated relative lateral and longitudinal positions provided by tracking module. Subsequently, advisory or imminent warnings are then generated for them by collision detection algorithm. The lateral and longitudinal distances are denoted by $D_{\text {lat }}$ and $D_{\text {lon }}$ and computed based on the projection of VRU position on the path of the vehicle. The distance between the VRU and projected point is $D_{\text {lat }}$ and the distance between the projected point and vehicle is $D_{\text {lon }}$. As it is shown in Figure 4-4-a, since in straight roads the vehicle heading is in the same direction with its path, the $D_{\text {lat }}$ and $D_{\text {lon }}$ are equal to $Y_{V R U}$ and $X_{V R U}$, respectively. On the other hand, for curved paths, such as curved roads or turning maneuvers, the heading is 
tangent to the curve of vehicle path as it is shown in Figure 4-4-b. The radius of this curvature $(R)$

is derived by $R=\frac{\psi}{v}$, where $\psi$ and $v$ are yaw rate and velocity, respectively. The intercept of predicted path of VRU and the curve of vehicle path is afterwards used to calculate the lateral and longitudinal distances.

\subsubsection{Congestion Control and Power Consumption Control Mechanisms}

Although the effect of congestion and power consumption issues is not visible in proof-of-concept and prototypes of V2P systems, these two issues along with smartphone positioning inaccuracy are the main challenges in front of realizing a large-scale ubiquitous V2P safety system.

Congestion, which is also investigated in V2V safety systems as the scalability problem, happens when multitudinous communication devices concurrently utilize the wireless channel in the range of each other. As a result, the channel throughput drops drastically, if the rate and range of transmissions by these devices are not properly controlled. Although V2V congestion control has been extensively investigated in the literature, such solutions may not be completely applicable to V2P systems due to the unforeseeable and fast movement patterns of pedestrians. Furthermore, the level of pedestrian density in a specific area could be higher by orders of magnitude in comparison with vehicles.

Power consumption, which primarily happens due to the high battery usage rate of GPS receivers and communication radios, is an exclusive issue of smartphones. Therefore, power consumption control mechanisms are required to optimize battery usage for V2P systems.

In our power consumption control approach, we first leverage the context awareness capability of smartphones to minimize the active duty cycle of the GPS receiver. Therefore, based on the currently available context information the GPS receiver is turned on or off. Whenever the device is in any of the following conditions the GPS should be turned off:

- Stationary (no Gyro variations)

- Indoor (inside building area or no GPS signal)

- Inside vehicle (moves as fast as the vehicle)

- Hiking trails, parks, and areas with no nearby vehicles

The change of these conditions is also detectable by smartphones. Moreover, the communication radios should also be turned off in these conditions, which also helps the congestion problem. Furthermore, the GPS duty cycle could be adjusted based on the required position update rate, which is controlled by our congestion control.

Our congestion control approach adaptively adjusts the transmission rate not only based on the explained context awareness scheme but also considering the surrounding conditions such as existence and distance of an approaching vehicle. Therefore, whenever the above-mentioned context conditions are not met, the smartphone should always listen to the channel and receive BSMs from vehicles. Furthermore, it should start the frequent transmission of its state information 
if an approaching vehicle is detected. Additionally, transmission range should also be controlled with the same criteria. For example, the VRU may reduce its transmission range if it senses many VRUs and few vehicles in its surroundings in order to form clusters and mitigate congestion. Since the location estimation of faster VRUs is more error-prone, the faster VRUs should transmit with a higher rate and range. For instance, a cyclist should transmit its position information farther and more frequent in comparison with a pedestrian. However, exceptions should be made for specific users at higher risk such as public safety and road workers. An evaluation of the proposed congestion mitigation method using ns-3 is presented as a part of the following section.

\subsection{Outdoor Test and Evaluation}

In this section, we implemented and evaluated the proposed V2P framework with a DSRCequipped Hyundai-Kia Sonata and a smartphone which had been DSRC-enabled by Qualcomm (Figure 4-1). Due to occupational safety considerations, in all of our tests, the smartphone was placed on top of a tripod with LOS of vehicle to resemble a stationary pedestrian, except the proofof-concept demonstration (Figure 4-5), which was performed with a real moving pedestrian and a slow speed vehicle $(30 \mathrm{~km} / \mathrm{h})$. The tests were conducted in a small parking lot with square size of approximately $500 \times 100 \mathrm{~m}^{2}$, in West Virginia University campus. The fastest scenario was around $70 \mathrm{~km} / \mathrm{h}$, which gave the safety system around 10 seconds of travel time at 200 meters of distance. This travel time was sufficient for generation of both advisory and imminent warnings (Figure 4-5). Different vehicle speeds were tested to study path prediction and position estimation.

In these tests, the transmission power was set to $10 \mathrm{dBm}$ and $20 \mathrm{dBm}$ for smartphone and WSU, respectively. These power values give roughly 500 meters of two-way situational awareness range.

The driver reaction time was set to $T_{D R D}=2.5 \mathrm{~s}$ and the maximum braking deceleration $d_{\max }=$ $-5.308-0.086 \times v_{b r k}$ [142]. Moreover, the other two parameters of the application are set as follows $T_{\text {gaurd }}=1 \mathrm{~s}$ and $T_{\text {mod }}=2 \mathrm{~s}$. 


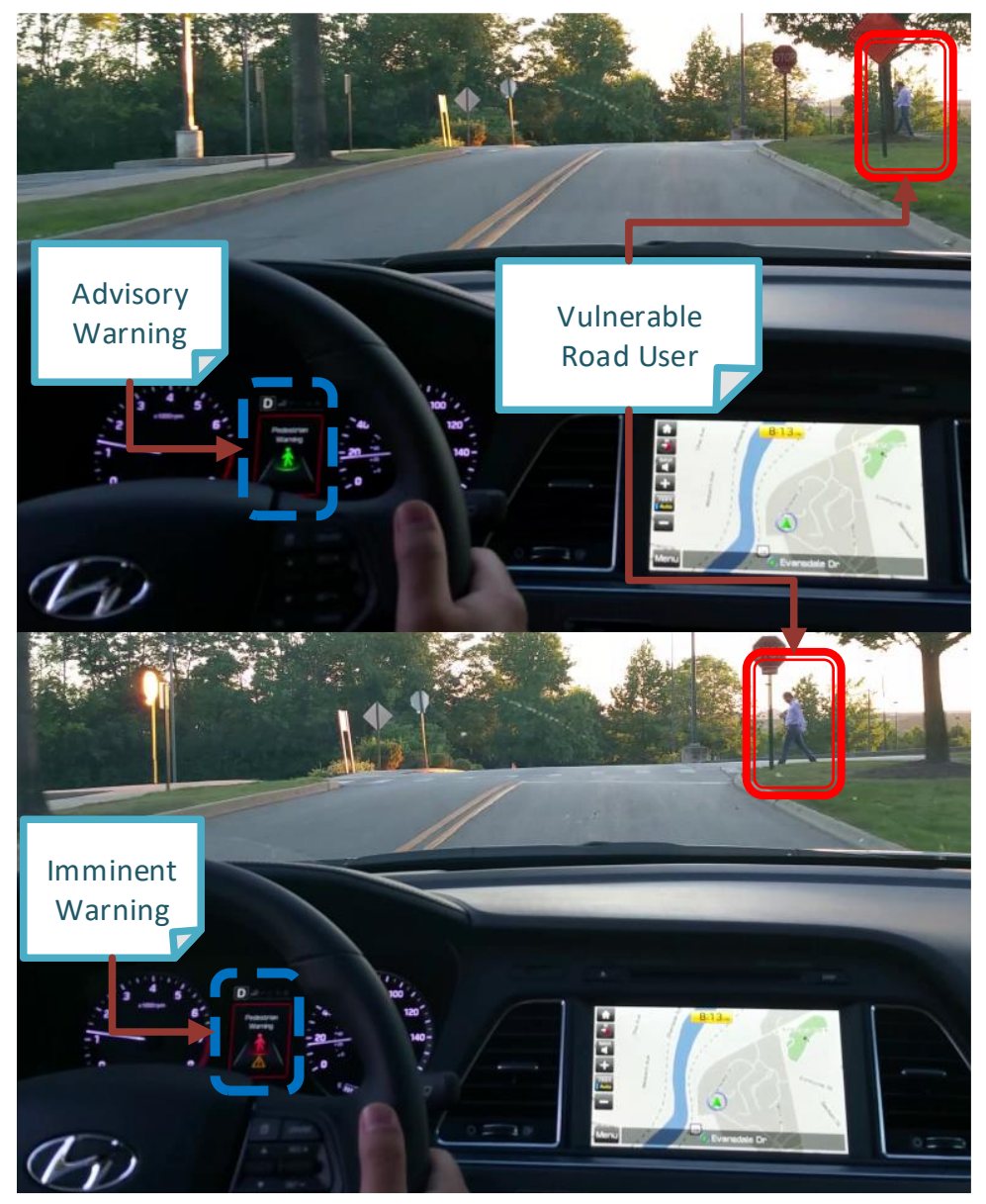

Figure 4-5- Proof-of-concept test of our cooperative VRU safety framework

Figure 4-6 illustrates the relative path of the smartphone from vehicle's real-time map perspective in a straight going vehicle scenario. Since the ENU coordinate system of the vehicle is always originated at the center of the vehicle, even though the smartphone is stationary, the vehicle observes it as if it is moving in the reverse direction. Different colors are used to draw the location of a smartphone, whenever it is classified inside one of the aforementioned zones. 


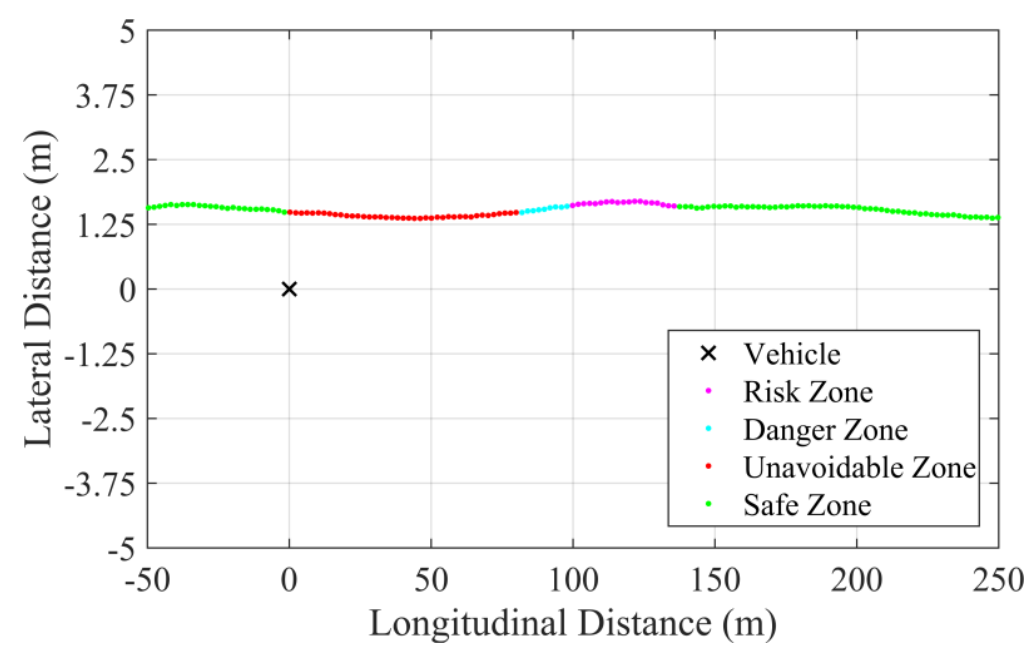

Figure 4-6- Relative path of the smartphone from the vehicle perspective.

The same scenario from the smartphone point of view is also shown in Figure 4-7. Similarly, whenever the smartphone detects itself inside one of the zones in front of the vehicle, it shows the location of the vehicle with the color of that zone.

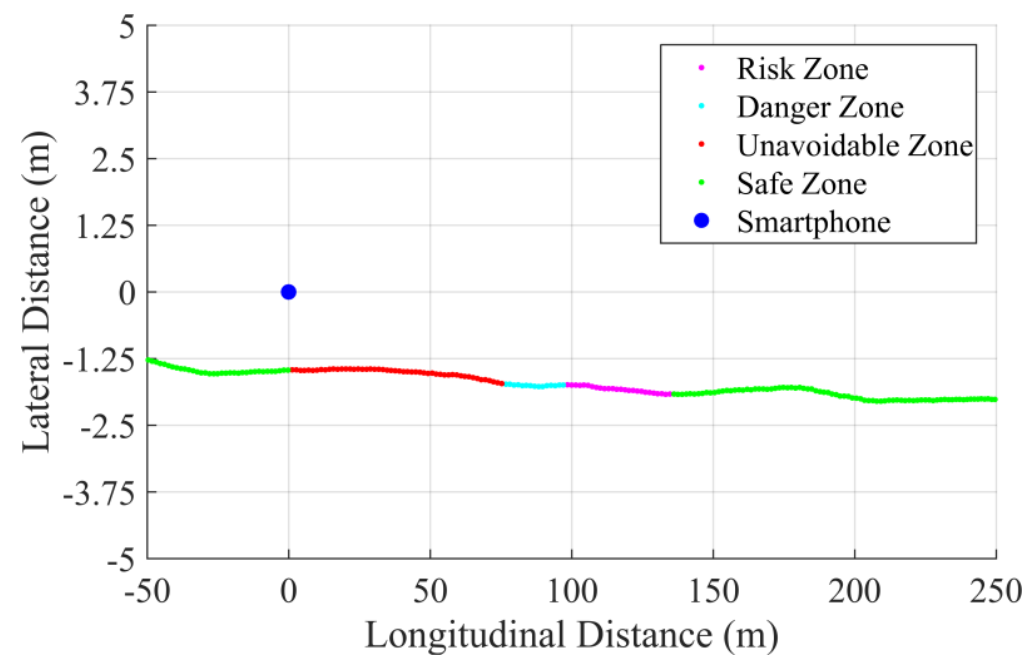

Figure 4-7- Relative path of the vehicle from the smartphone perspective.

The corresponding distance-to-stop of each zone along with the longitudinal distance of vehicle and smartphone for the whole scenario duration is depicted in Figure 4-8. The advisory and imminent warning generations are initiated whenever the $D_{\text {lon }}<D T S_{\text {mod }}$ and $D_{\text {lon }}<D T S_{\text {guard }}$ conditions are met for the first time, respectively. 


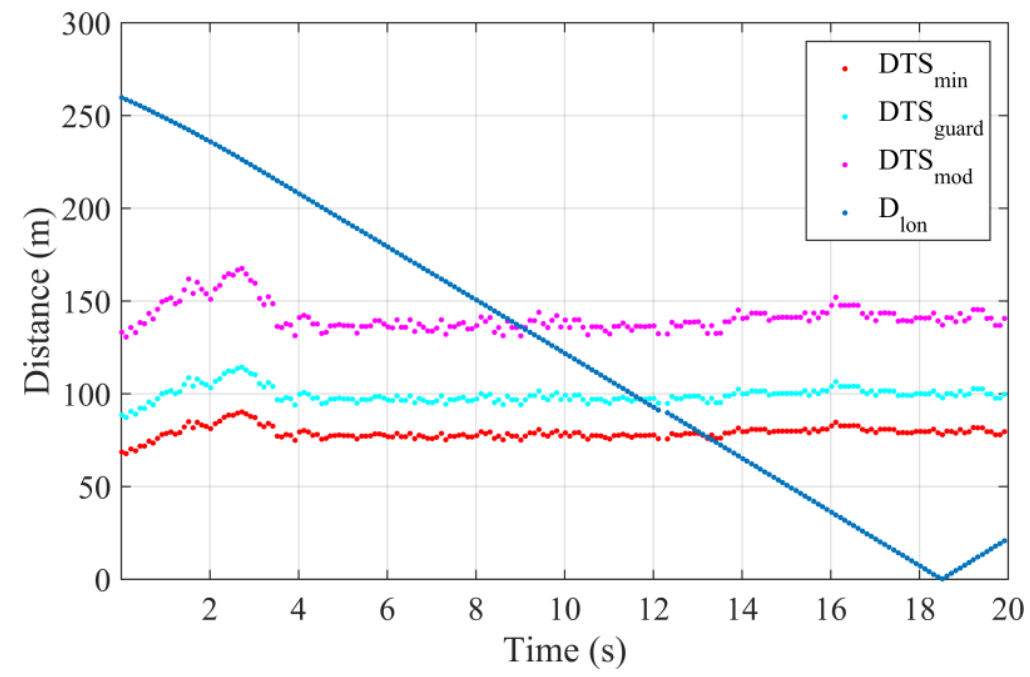

Figure 4-8- Corresponding distance-to-stop of each zone along with the longitudinal distance of vehicle and smartphone.

Equivalent figures for the curved road scenario are shown in Figure 4-9, Figure 4-10, and Figure 4-11.

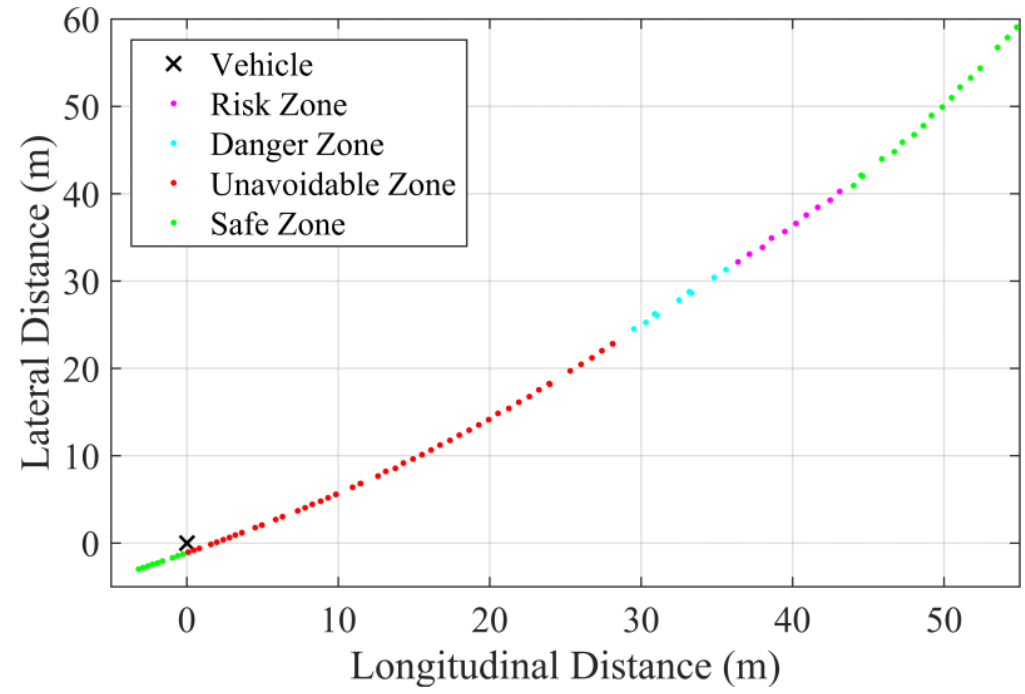

Figure 4-9- Relative path of the smartphone from the vehicle perspective. 


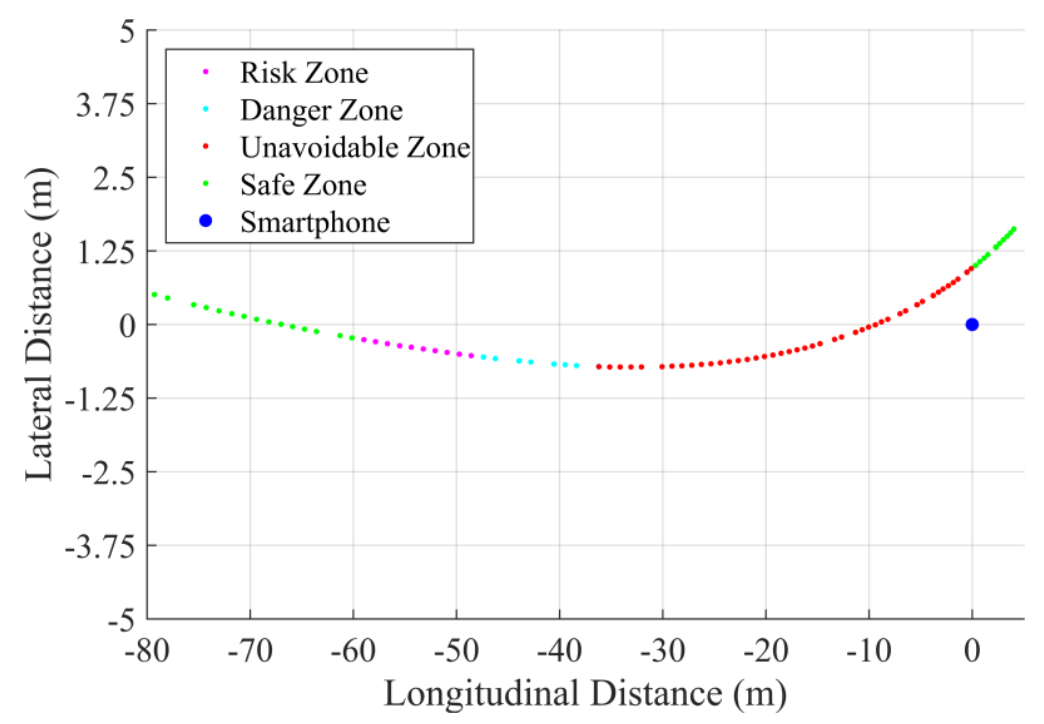

Figure 4-10- Relative path of the vehicle from the smartphone perspective.

Since distance-to-stop is a function of current speed and acceleration, its values are changing in a decreasing manner as the vehicle brakes to stop by the smartphone in the curved road scenario in Figure 4-11.

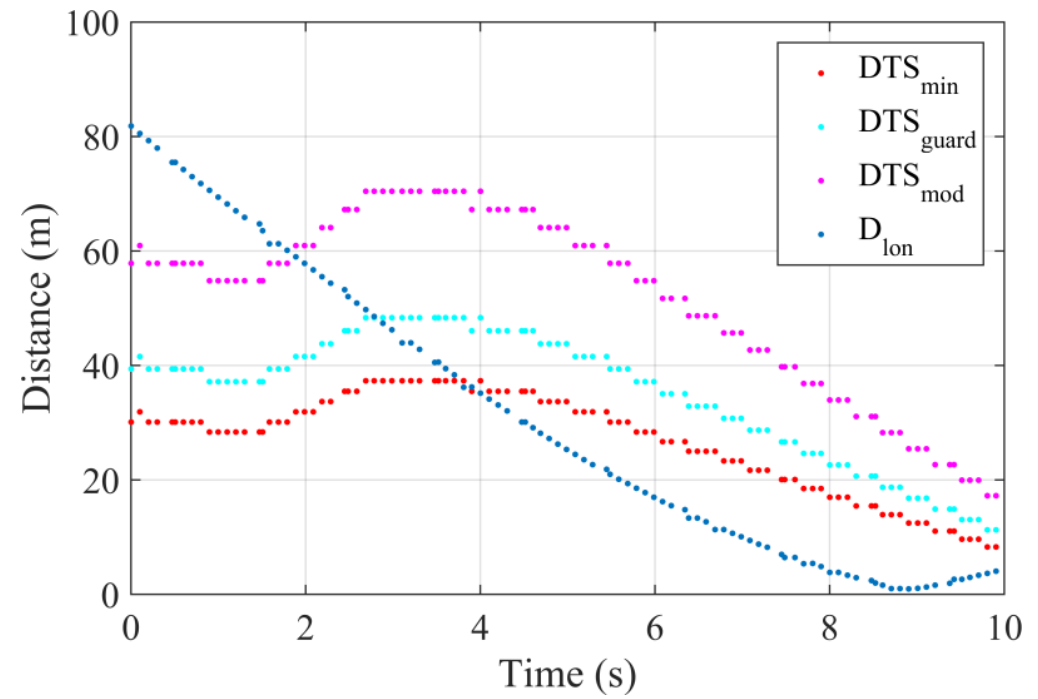

Figure 4-11- Corresponding distance-to-stop of each zone along with the longitudinal distance of vehicle and smartphone.

Furthermore, we use ns-3, which is a multipurpose full-stack network simulator, to evaluate the performance of our congestion mitigation mechanism. The ns-3 includes a comprehensive implementation of DSRC standards. In our simulations, the PHY layer is configured based on 802.11p protocol with $10 \mathrm{MHz}$ bandwidth and OFDM bit rate of $6 \mathrm{Mbps}$. Other constant parameters are summarized in Table 4-1. In order to study the performance of the proposed context awareness mechanism, the transmission rate and range are fixed at $10 \mathrm{~Hz}$ and 500 meters, respectively. In the 
designed congestion scenario, 400 pedestrians are randomly distributed on both sides of a 1200 meters unsignalized straight road and moving across the sidewalks, while 4 vehicles drive from one end to the other.

Table 4-1- ns-3 Simulation Parameters

\begin{tabular}{l|r||l|r}
\hline \hline \multicolumn{1}{c|}{ Parameter } & \multicolumn{1}{|c|}{ Value } & \multicolumn{1}{c}{ Parameter } & Value \\
\hline \hline No. Pedestrians & 400 & AIFSN & 7 \\
\hline No. Vehicles & 4 & $\begin{array}{l}\text { Contention } \\
\text { Window }\end{array}$ \\
\cline { 2 - 4 } $\begin{array}{l}\text { Transmission } \\
\text { Rate }\end{array}$ & $10 \mathrm{~Hz}$ (Fixed) & $\begin{array}{l}\text { Simulation } \\
\text { Time }\end{array}$ & $50 \mathrm{sec}$ \\
\hline Range & 500 meters & $\begin{array}{l}\text { Transmission } \\
\text { Power }\end{array}$ & $15 \mathrm{dBm}$ \\
\hline \hline
\end{tabular}

The scenarios with and without congestion control are compared in terms of Packet Error Ratio (PER) in Figure 4-12. Note that the recorded PER versus distance is averaged for each device, smartphones, and vehicles, over the entire simulation time.

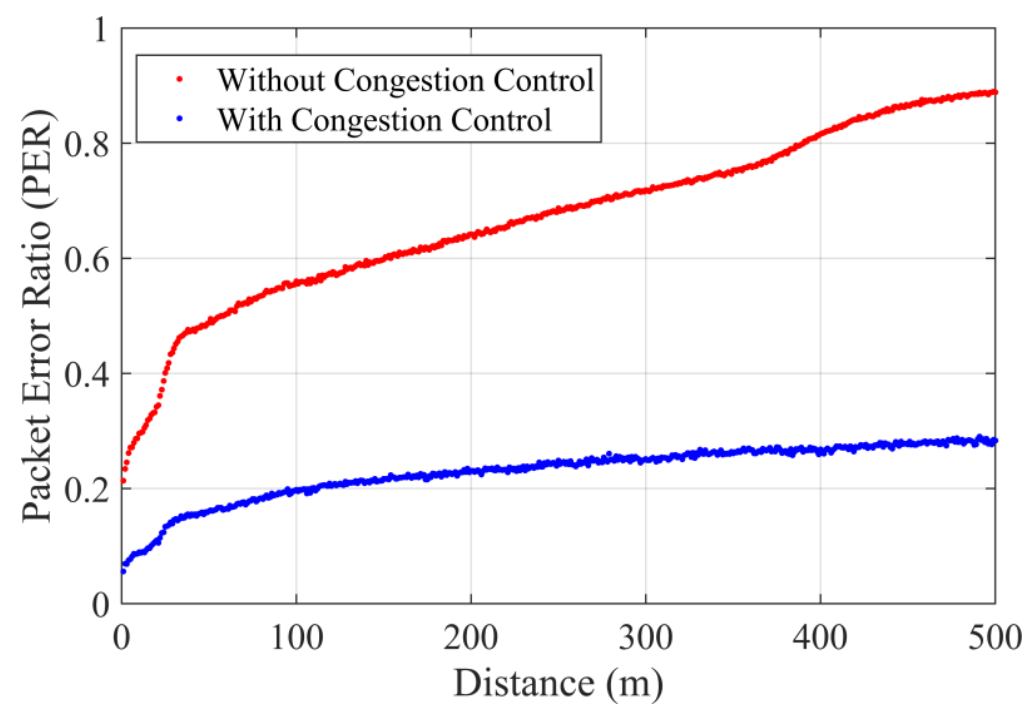

Figure 4-12- Congestion control performance in terms of PER versus distance.

Channel Busy Percentage (CBP) is also recorded in each device during the simulation time. The averaged CBP versus time for both scenarios is shown in Figure 4-13. 


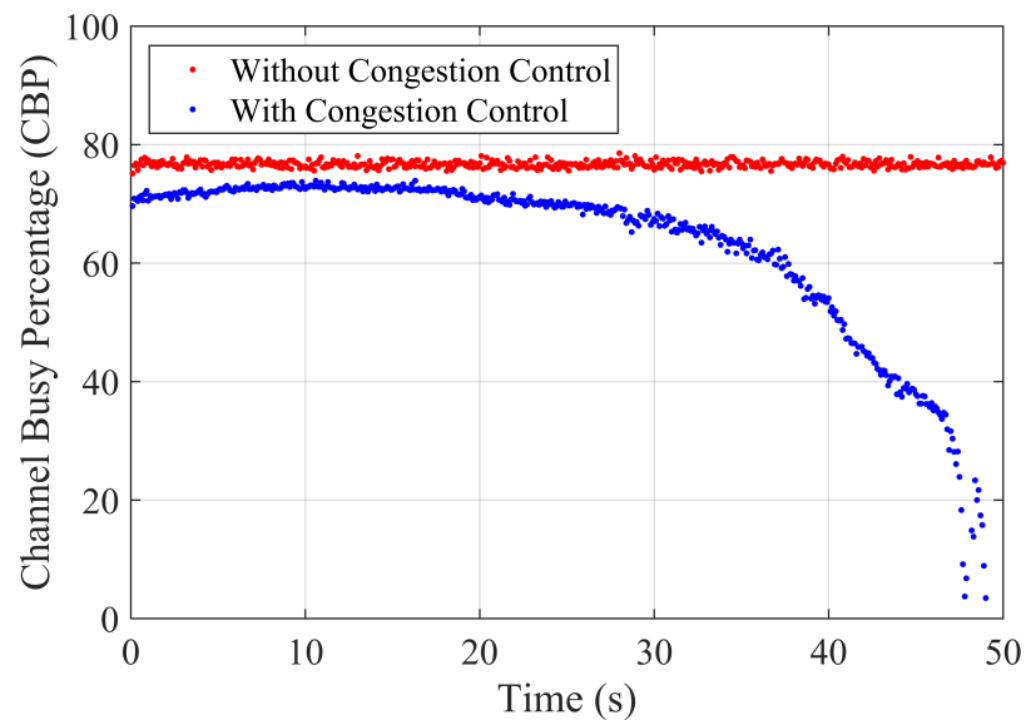

Figure 4-13- Averaged CBP versus time for with and without congestion control scenarios. 


\section{Chapter 5 Situational Awareness Enhancement with Learning-based Driver Behavior Modeling}

Each maneuver of a vehicle is an immediate and direct consequence of its driver's intention. The realization of this intention happens using a specific set of mechanisms, such as steering wheel, pedals, and handles. The utilization of these tools can be directly measured through time-varying patterns of vehicle dynamic parameters such as steering wheel angle, throttle, brake pedal position, lateral position, longitudinal position, velocity, acceleration, heading, yaw rate, and signaling status. However, it is not possible to deterministically assign a maneuver to a specific pattern of these parameters as different maneuvers may have partially similar sections. Therefore, a comprehensive approach is required to discriminate different driving maneuvers based on measured patterns of their parameters.

Among unexpected vehicle maneuvers, a unsignaled lane change is one of the most dangerous scenarios. Between lane change scenarios, cut-out maneuvers to an empty lane are generally safe and can be compensated without any risk. For example, in a CACC platoon, the vehicle behind the leaving vehicle only needs to catch up with its new leader to fill the cut-out gap and the same behavior will then propagate through the rest of the platoon. In contrast, cut-in maneuvers require a more precise tracking and observation as they increase the risk of accidents. Thus, it is welldesired to predict cut-in intention of the driver beforehand to prevent threatening situations.

We specifically concentrate on cut-in maneuvers for CACC platoons, as a vehicle in a stable CACC platoon has to hard brake when another vehicle cuts into the platoon just in front of it. This maneuver can lead to platoon deformation and even chain crash. Therefore, anticipating the cut-in maneuver of the neighboring vehicle and predicting the new future behavior should be an inseparable part of CACC and platooning system design.

Leveraging actual and accurate information of neighboring vehicles through VANETs, by which vehicles communicate their parameters periodically, we can predict driver behavior of a cut-in suspicious vehicle in the adjacent lane. Note that, although the provided dynamics of the adjacent vehicles via communication are noise-free compared to local sensory data, the tradeoff is the 
freshness of such information which is affected by communication latency and packet loss. Therefore, the accuracy of the driver behavior model heavily relies on the network conditions.

We use time series based approaches for our driver behavior modeling and trajectory prediction. A combination of nonlinear autoregressive (NAR), nonlinear autoregressive exogenous (NARX), and recurrent neural networks (RNN) are then trained and used to this end. The outcome of the prediction model is then mapped to an unsafe lane change probability function which is used by a stochastic model predictive controller (Stochastic-MPC) to achieve three primary objectives. First, the controller in each vehicle should minimize the spacing error between the vehicle and the immediate ahead vehicle, while keeping their velocity difference as close as possible to zero. Concurrently, it must respond appropriately to a cut-in maneuver.

\subsection{System Descriptions}

In our framework, the vehicle inside the platoon, which directly affected by the cut-in suspicious vehicle, is referred to as the host vehicle. The immediate vehicle in front of the host vehicle is known as the preceding vehicle, and the first vehicle of the platoon is the leading vehicle or leader. The dangerous area in front of the host vehicle is referred to as bad-set. Although, detection of cutin by the vehicle itself is beneficial to some applications such as lane keep assist system (LKAS) and blind spot warning (BSW), CACC and platooning need the lane change maneuver to be detected remotely by the host vehicle. Therefore, for the CACC platoons, the behavior of the cutin vehicle will be predicted and modeled by the host vehicle and not by itself. The remote lane change detection is required because the host vehicle should react in a timely manner to avoid hazardous situations. V2V communication periodically provides the parameters of the cut-in vehicle through basic safety messages (BSM).

In our model, we assume that the host vehicle, which is in a stable condition in the platoon, periodically receives the BSMs of its surrounding vehicles and continuously traces them prior to any probable cut-in maneuver. From BSM part one of the SAE J2735 standard, we utilize the following parameters for our behavior modeling: Latitude, Longitude, Elevation, Speed, Heading, Steering Wheel Angle, 4-Way Acceleration Set, and Vehicle Size. The latitude, longitude, and elevation represent the location of the center of the vehicle in the WGS-84 coordinate system. The 4-way acceleration set consists of acceleration values in 3 orthogonal directions plus yaw rate, which are calculated based on the assumption that the front of the vehicle is the positive longitudinal axis, the right side of it is the positive lateral axis and clockwise rotation as the positive yaw rate.

In CACC platooning, a safe longitudinal gap must be continuously kept between each two consecutive vehicles. The deviation from the safe gap, which is known as a spacing error, should remain as small as possible to reduce the risk of collision and take advantage of platoon formation, such as lower fuel consumption and higher traffic throughput. As mentioned, bad-set is the dangerous area in front of the vehicle in which the safe gap is violated. In other words, bad-set is a rectangle aligned with the road surface in front of the host vehicle, while its longitudinal dimension depends on the platoon speed and is equal to the desired longitudinal safe gap and its lateral dimension is the lane width. Bad-set longitudinal and lateral dimensions are denoted by $L_{b s}$ and $W_{b s}$, respectively. The front bumper of the host vehicle is always located at the center of the bad-set rear lateral edge (Figure 5-1). 


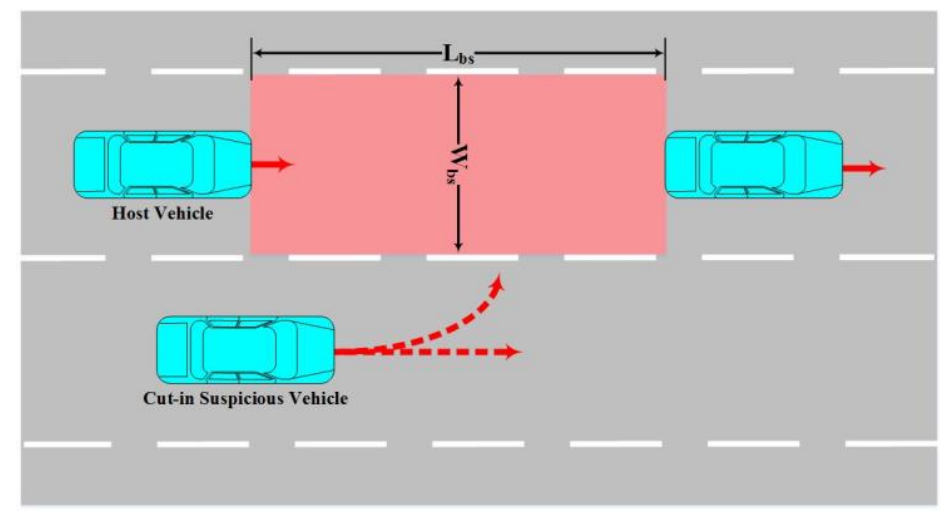

Figure 5-1- Host vehicle bad-set and prospective cut-in maneuver

The goal of the behavior modeling component is to track and predict the trajectory of all of the vehicles in the adjacent lanes of the host vehicle. The model should not only predict the immediate kinematics of the vehicles but also the complete high-level driving maneuvers. Therefore, the position of neighboring vehicles should be predicted for multiple steps based on their current and previous communicated information. The number of required prediction steps is determined by the duration of a complete high-level maneuver and denoted by $S_{m}$. This multi-step prediction is then used to determine the probability of unsafe lane change which is passed to the Stochastic-MPC for better estimation of required spacing gap between two vehicles.

\subsubsection{Problem Statement of Lane Change Maneuver}

Each lane change maneuver consists of four separate phases: Intention phase, Preparation phase, Transition phase, and the Completion phase. The driver who finds enough gap between two vehicles and decides to change its lane is in the intention phase. The intention phase does not have any physical action and the driver only evaluates the possibility of the lane change and assesses the risks of the lane change [143]. The evaluation is subjective and heavily depends on the benefits of this lane change for the driver, his estimation of the behavior of adjacent lane vehicles, the driving habits of the driver, and the prediction of required acceleration and speed for a safe lane change [144]. The preparation phase commences whenever the driver decides to perform the lane change. This phase consists of the adjustment of the vehicle in its current lane to leave enough gap for the lateral movement of next phase. The physical action of the driver in the preparation phase is longitudinal acceleration or deceleration. In the next phase, after the preparation phase adjustments, the lateral acceleration and movement of the vehicle begin to shift into the targeted adjacent lane. The transition phase starts by noticeable lateral acceleration and continues by a lateral deceleration when the vehicle has reached the intended lane. The lateral acceleration and lateral speed are bounded by the comfortable lateral acceleration threshold and the maximum tolerable lateral speed, respectively [145]. To safeguard a smooth transition of the vehicle between lanes, the longitudinal acceleration is bounded by $-0.8 g$ and $2 g$. The last phase is the completion phase in which the vehicle adjusts its position and speed to stay at the center of the lane and uphold a safe gap from its front and behind vehicle. Therefore, our model is designed to not only predict the immediate kinematics of the vehicles in the transition phase but also the complete driving maneuvers from beginning to end. 
Received BSMs from surrounding vehicles are used to convert their coordinates to a rotated EastNorth-Up (ENU) coordinate system with respect to the position and heading of the host vehicle. The $x$ and $y$ coordinates show the relative longitudinal and lateral positions of each neighboring, respectively. The trajectory of each remote vehicle is then modeled as a time series, in which the sequence of received position information from BSMs is considered as the time dependent variable set. In our model, we separate the learning of lateral and longitudinal trajectories of cut-in suspicious vehicle as they are influenced by different control inputs. Moreover, the significant part of longitudinal behavior is performed in the preparation phase prior to start of lateral movement.

By definition, a time series is a sequence of data ordered in time. It is implicitly assumed that the future value of a time series is related to its past and current values. Therefore, prediction methods are used to estimate the future value of the time series based on its observed values. If the undiscovered underlying system that generates a time series is deterministic, prediction models can determine the unknown system equations and estimate the future state of the system. On the other hand, if the underlying system is stochastic, prediction models will characterize the probability distribution function (pdf) of the underlying stochastic system and can approximate the future state based on it. Many linear models such as autoregressive (AR), moving average (MA), and autoregressive exogenous (ARX) have been vastly used for time series prediction; however, they fail to capture the behavior of nonlinear systems. Lane change maneuver which involves a stretch of different driving decisions is an example of such nonlinear models. Therefore, nonlinear time series prediction models are needed to characterize such systems. Artificial neural networks (ANNs) are one of the most famous prediction tools for description and prediction of nonlinear systems and it has been shown that neural networks with hidden units can principally predict any well-behaved function [146]. In this work, we used nonlinear autoregressive (NAR), nonlinear autoregressive exogenous (NARX) and recurrent neural networks (RNN) toward driver behavior and lane change prediction.

\subsection{Neural Networks for Driver Behavior Modeling}

As mentioned, ANNs can characterize and learn nonlinear relationships and patterns between inputs signals and outputs. In the case of time series, in order to handle the dependency of the prediction to a finite set of past values and time varying nature of the input signals, neural network topologies need to be equipped with a short-term memory mechanism which is called the feedback delay. In this work, we use three different types of ANNs with feedback delays, namely NAR, NARX, and RNN.

NAR is a neural network with feedback delay that can be trained and used to predict a time series only from its past values and does not rely on any external inputs. We use NAR model to predict the pattern of input signals to the system, based on the driver behavior. Therefore, the driver behavior model is captured through NARs from the input signals to the vehicle system, which are steering wheel angle, heading, speed, and longitudinal acceleration.

If an exogenous time series that affects the outcome of this time series is available as an external input, another nonlinear model should be used. NARX is a neural network with feedback delay that can be trained and used to predict a time series from its past values and an exogenous one. We use NARX model to predict the longitudinal trajectory of the vehicle during the lane change using some of the previously estimated sequences of input signals as the exogenous input. Therefore, the 
longitudinal motion of the vehicle is modeled using the predicted values of heading, speed, and longitudinal acceleration as external input signals to the system.

Finally, an RNN is adopted to model the lateral trajectory of the vehicle based on the predicted input signals. RNNs are similar to feedforward neural networks, except that each layer has a recurrent feedback delay connection linked to it. These recurrent connections allow the RNN to have an infinite dynamic response to input data of time series, which is different from NARX and NAR as they have finite input responses. The infinite dynamic response creates an internal state for the RNN which allows it to manifest dynamic temporal behavior. Therefore, RNNs can use their internal memory to process arbitrary sequences of inputs. The input signals to our lateral position prediction RNN are steering wheel angle and heading. Using the internal memory, the RNN can distinguish between different maneuvers with partially similar input signals. For example, a steering due to the road curvature might look similar to the one from lane change maneuver, but the RNN learns to distinguish between these two maneuvers by looking at other input signals.

All of the ANN models are batch trained and the training phase is offline. The low computational cost of batch training and insufficiently accessible data for online training are the main reasons for this choice. The details of the training and performance results will be discussed in the evaluation section.

\subsection{CACC Controller Design}

Considering a CACC platoon of $n$ vehicles, the spacing error of the $i$-th following vehicle is defined as [147]

$$
\delta_{i}=x_{i-1}-x_{i}-h v_{i}-L_{i}-d_{0}
$$

for all $i \in\{1,2, \ldots, n\}$, where $x_{i}$ and $v_{i}$ are longitudinal position and velocity of the $i$-th following vehicle and $x_{0}$ stands for the longitudinal position of the lead vehicle; $h$ is the time gap, $L_{i}$ is the length of the vehicle and $d_{0}$ is the minimum desired distance between each vehicle and its preceding vehicle in the platoon. Then, the dynamics of the $i$-th following vehicle is modeled as follows

$$
\begin{gathered}
\dot{\delta}_{i}=v_{i-1}-v_{i}-h \dot{v}_{i} \\
\Delta \dot{v}_{i}=a_{i-1}-a_{i} \\
\dot{a}_{i}=f_{i}\left(a_{i}, v_{i}\right)+g_{i}\left(v_{i}\right) c_{i}
\end{gathered}
$$

where the control input $c_{i}$ represents the throttle of engine for $c_{i}>0$ and the brake input for $c_{i}<$ $0, a_{i}$ is the acceleration of the $i$-th vehicle, and $f_{i}$ and $g_{i}$ are nonlinear functions which represent the longitudinal dynamics of the vehicle. After selection of the proper control law for $c_{i}$ to achieve feedback linearization, the third equation in (5.2) can be rewritten as

$$
\dot{a}_{i}(t)=-\frac{a_{i}(t)}{\zeta_{i}}+\frac{u_{i}(t)}{\zeta_{i}}
$$

where $u_{i}$ is an input signal which will be designed using a model predictive controller (MPC). However, due to the communication delay the acceleration value of preceding vehicle reaches the 
host vehicle with a delay. Denoting the communicated acceleration of $i$-th vehicle at receivers by $\bar{a}_{i}(t)$, the state space equation for a vehicle in a CACC system is derived as follows

$$
\dot{x}_{i}(t)=A_{i} x_{i}(t)+B_{i} u_{i}(t)+G_{i} \bar{a}_{i-1}(t)
$$

with state vector of

$$
x_{i}=\left[\begin{array}{lll}
\delta_{i} & \Delta v_{i} & a_{i}
\end{array}\right]^{T}
$$

and

$$
A_{i}=\left[\begin{array}{rrr}
0 & 1 & -h \\
0 & 0 & -1 \\
0 & 0 & -\frac{1}{\zeta_{i}}
\end{array}\right] \quad B_{i}=\left[\begin{array}{l}
0 \\
0 \\
1 \\
\zeta_{i}
\end{array}\right] \quad G_{i}=\left[\begin{array}{l}
0 \\
1 \\
0
\end{array}\right]
$$

\subsubsection{Model Predictive Controller for CACC}

An MPC is designed for the system presented in (5.4) to achieve three primary objectives. The controller should compute the input signal $u_{i}$ to minimize the spacing error while keeping the velocity as close as possible to the velocity of preceding vehicle. Concurrently, it must respond appropriately to a cut-in maneuver. In order to obtain an optimal control problem, the continuous time dynamics of the system is discretized using the Euler forward method with a time step $T_{s}$ :

$$
x_{i}[k+1]=A_{i}^{k} x_{i}[k]+B_{i}^{k} u_{i}[k]+G_{i}^{k} \bar{a}_{i-1}[k]
$$

where

$$
A_{i}^{k}=\left[\begin{array}{ccc}
1 & T_{s} & -h T_{s} \\
0 & 1 & -T_{s} \\
0 & 0 & 1-\frac{T_{s}}{\zeta_{i}}
\end{array}\right] \quad B_{i}^{k}=\left[\begin{array}{c}
0 \\
0 \\
\frac{T_{s}}{\zeta_{i}}
\end{array}\right] \quad G_{i}^{k}=\left[\begin{array}{c}
0 \\
T_{s} \\
0
\end{array}\right]
$$

Then the cost function of the optimization problem with a control horizon $N$ is defined based on the primary objectives of the controller

$$
J[k]=\sum_{n=1}^{N} c_{\delta} \delta_{i}^{2}[k+n]+c_{v} \Delta v^{2}[k+n]+c_{u} \Delta u^{2}[k+n]
$$

with $c_{\delta}, c_{v}$, and $c_{u}$ are weighting coefficient reflecting the relative importance of each term and

$$
\Delta u[k+n]=u[k+n]-u[k+n-1]
$$

which is an extra term added to the cost function to prevent a large variation of the input signal to the CACC system since having a smooth input signal is interpreted as a comfort ride. Then the output of the controller at each time step is the solution to the following optimization problem: 


$$
\begin{aligned}
& \min _{u} J[k] \\
& \text { s.t. } \\
& u_{\min } \leq u[k+n] \leq u_{\max } \\
& v_{\min } \leq v[k+n] \leq v_{\max }
\end{aligned}
$$

The driver behavior model from the previous section, which predicts the future lateral and longitudinal acceleration of all neighboring vehicles, is then used to solve the optimization problem. The model updates the input signals as soon as it receives a new BSM from the leading vehicle.

\subsubsection{MPC with Lane Change Prediction}

The MPC proposed above maximizes the throughput of a CACC platoon. However, in real traffic scenarios a vehicle might cut-in and enter the platoon unexpectedly so the host vehicle in the platoon must decelerate to a safe gap. Therefore, the controller should respond to a cut-in scenario.

The prediction performance of the ANNs is used to determine the confidence level of each future step prediction. The 90-percentile confidence interval of lateral and longitudinal positions is used for the cut-in probability calculation, which means that with 90 percent probability the predicted point is within this interval for each step. These intervals for each step are then used to calculate the probability of a suspicious vehicle entering the bad-set of the host vehicle in the CACC platoon. Considering both longitudinal and lateral confidence intervals, a rectangle around the predicted point shows the area in which the actual vehicle will be in the future. The most overlapping area of the set of confidence rectangles with bad-set divided by the area of that confidence rectangle and multiplied by the $90 \%$ confidence, shows the probability of having the cut-in vehicle in the bad-set of the host vehicle. Having this probability, we can redefine the spacing error as follows

$$
\delta_{i}=\frac{1}{2-e^{-\alpha P d c}}\left(x_{i-1}-x_{i}\right)-h v_{i}-L_{i}-d_{0}
$$

where $P_{d c}$ is the probability of the cut-in suspicious vehicle being in the bad-set of the host vehicle, and $\alpha$ is a constant control parameter which adjusts the reaction sensitivity of the MPC to a specific cut-in maneuver. When the probability of the predicted lane change is one, the controller doubles the distance to its current preceding vehicle by halving its distance in the spacing error equation. Consequently, the cut-in vehicle has enough room to enter the CACC platoon. On the other hand, when the probability is zero, which means that the suspicious vehicle is not going to cut-in or has safe distance from the host vehicle for its maneuver, the denominator of (5.12) becomes one and the host vehicle keeps the normal safe distance $h v_{i}+d_{0}$ to its preceding vehicle. Figure 5-2 shows the effect of different values of the control parameter $\alpha$ on spacing error coefficient. 


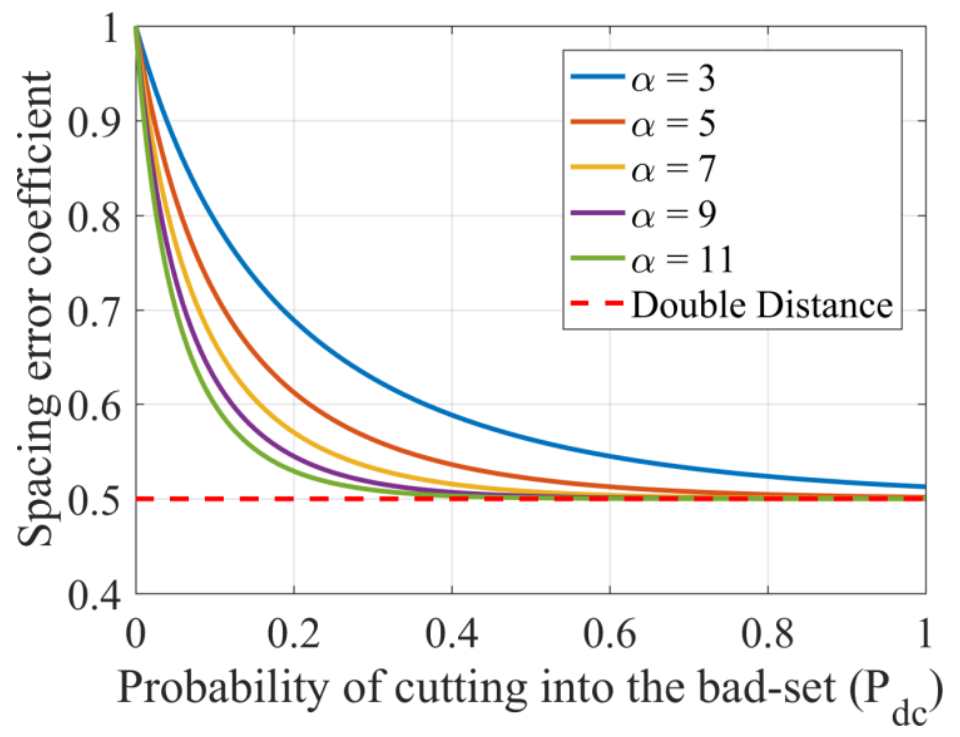

Figure 5-2-Effect of reaction sensitivity parameter $(\alpha)$ on spacing error

\subsection{Performance evaluation}

To evaluate the performance of our method, we extracted 90 lane change maneuvers from the BSMs generated by participating vehicles in the Safety Pilot Model Deployment (SPMD) in Ann Arbor, Michigan [148]. We first present the ANN training and testing process and performance, followed by the MPC results.

\subsubsection{Training, Testing, and Performance of ANNs}

The first phase of our neural networks training process is preprocessing and preparation. In order to use the full capability of neural networks, and since the activation function of each neuron is in the $[-1,1]$ interval, the input signals for all ANNs are normalized to this range. The second step toward the preprocessing is differencing the input signals. The differencing operation is used to remove the linearity in the input signals in order to improve the nonlinearity prediction process. The resulting time series is known as integrated time series. Once the ANN predicts the difference between any two consecutive values in the time series, the value of predicted location can be reconstructed by adding the first actual value to the estimated difference in the series. Subsequently, small variations of input signals, based on the nature of the signal, are removed to smooth the time series and mitigate the effect of noise. Variation smaller than 3 degrees, $0.1 \mathrm{rad}$, $0.1 \mathrm{~m} / \mathrm{s}$, and $0.1 \mathrm{~m} / \mathrm{s}^{2}$ are removed from steering wheel angle, heading, speed, and longitudinal acceleration, respectively. The resulting input signals during one maneuver is shown in Figure 5-3. Finally, the signals from all 90 maneuvers are concatenated to create a long univariate time series for each input signal. 


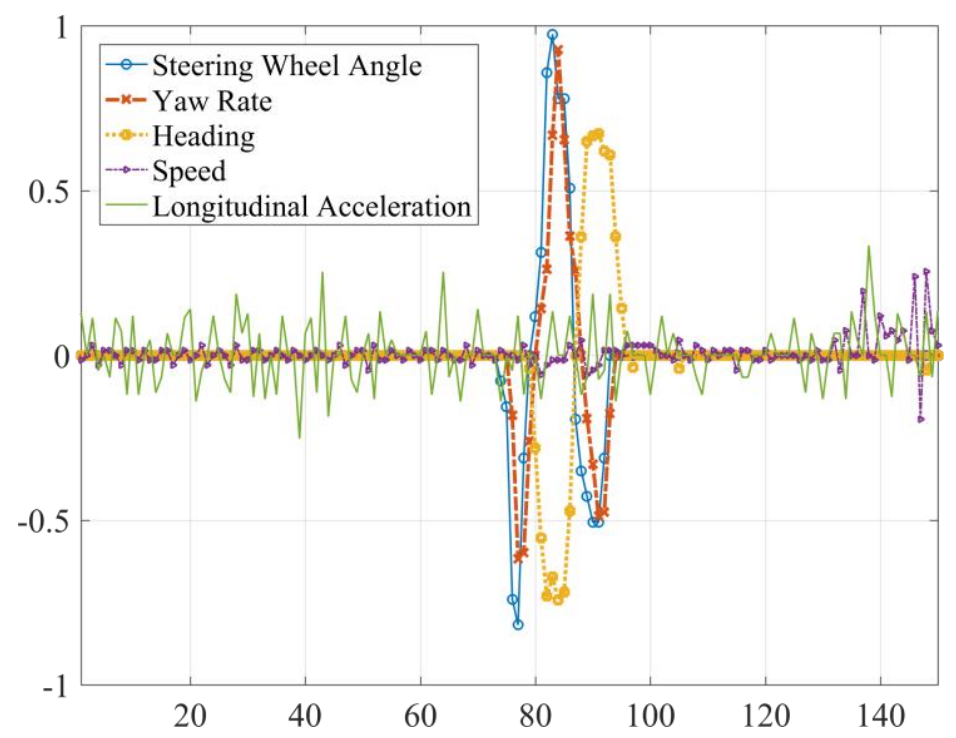

Figure 5-3- Smoothed, Normalized, and integrated input signals of a single lane change maneuver

All of our ANNs have 20 nodes in their hidden layer and 15 step short term memory, which means that they are using the past information of 1.5 seconds for future prediction. The required prediction steps are also set to 10 steps for all of the ANNs, $S_{m}=10$, which means that the we are predicting the behavior of the driver for 1 second in the future, since the driver can change its decision and behavior beyond this time. Finally, a 70-15-15 percent training, cross-validation, and testing data selection is used for ANNs training and performance evaluation.

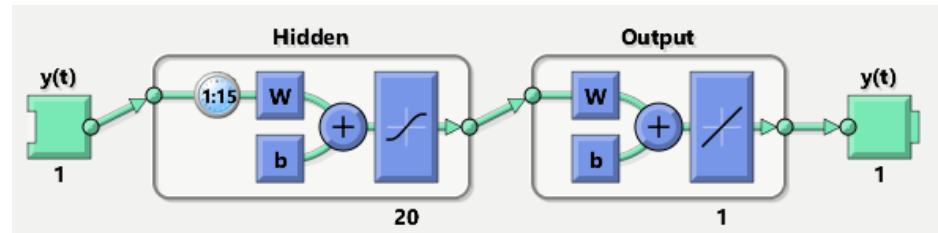

(a)

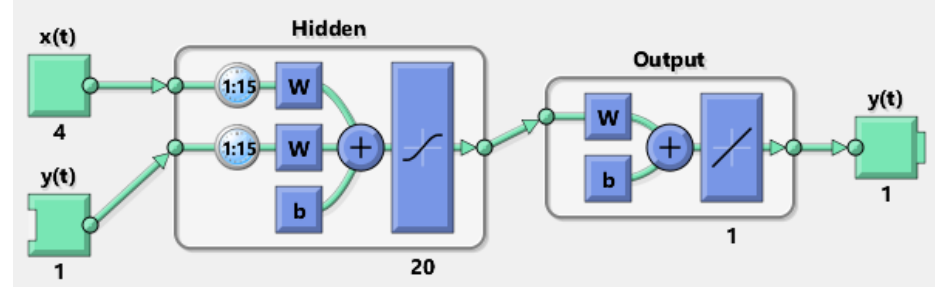

(b)

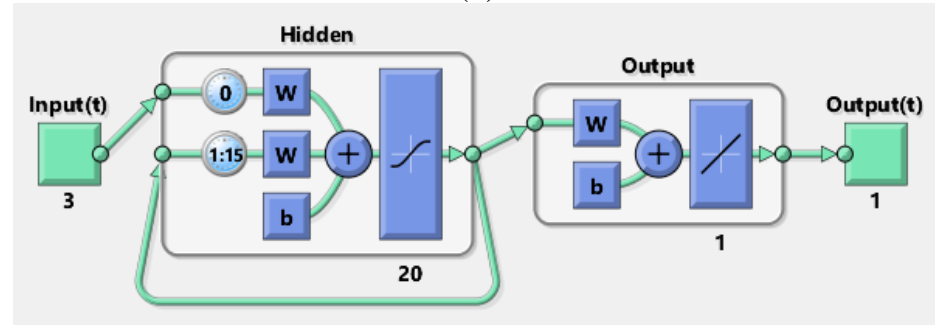

(c)

Figure 5-4- Layer structure of (a) NAR (b) NARX (c) RNN 
Figure 5-4 (a), (b), and (c) show the layer structure of our designed NAR, NARX, and RNN, respectively. As mentioned before, The NAR is used to model the patterns of input signals to the system. These patterns capture the general behavior of drivers during a lane change maneuver. The NARX and RNN are used to model and predict the longitudinal and lateral position of the vehicle, respectively. The longitudinal position of the vehicle is modeled based on the predicted values of heading, speed, and longitudinal acceleration as external inputs. Therefore, the NARX only tries to find the nonlinear relationship between these inputs and the future longitudinal position of the vehicle. On the other hand, the RNN should not only predict the future lateral position of the vehicle but should also distinguish between different lateral maneuvers. Therefore, the lateral model is also trained with some curve road data to be able to differentiate between different lateral movements.

The performance of two trajectory prediction methods, vehicle kinematic model, and our trained RNN, for a lane changing vehicle in terms of lateral position are shown in Figure 5-5. The classic car model has the following kinematic-based differential equations [149]

$$
\left\{\begin{array}{l}
\dot{x}_{i}=v_{i} \cos \theta_{i} \\
\dot{y}_{i}=v_{i} \sin \theta_{i} \\
\dot{\theta}_{i}=\frac{v_{i}}{L_{i}} \tan \phi_{i}
\end{array}\right.
$$

where $x_{i}, y_{i}$, and $v_{i}$ are longitudinal position, lateral position, and velocity of the $i$-th vehicle, respectively. Also $\phi_{i}$ denotes the steering angle and $L_{i}=5$ is the length of the vehicle.

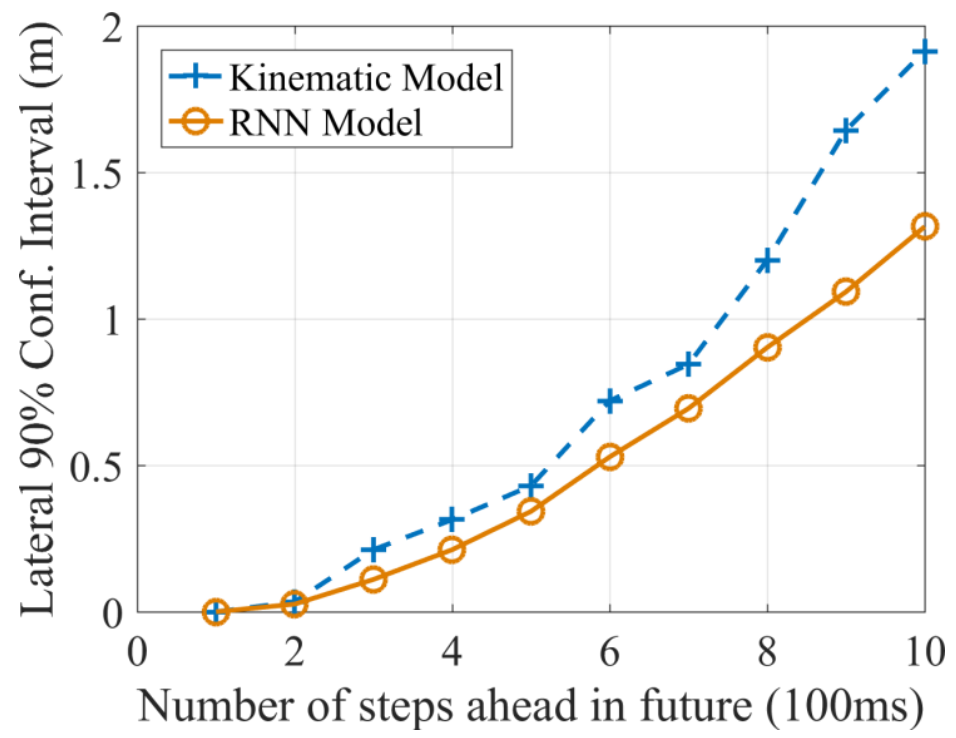

Figure 5-5- Comparison of 90-percentile conf. interval of Lateral position prediction of the Kinematic and RNN models for different prediction steps

Figure 5-6 shows the same comparison for longitudinal position prediction. 


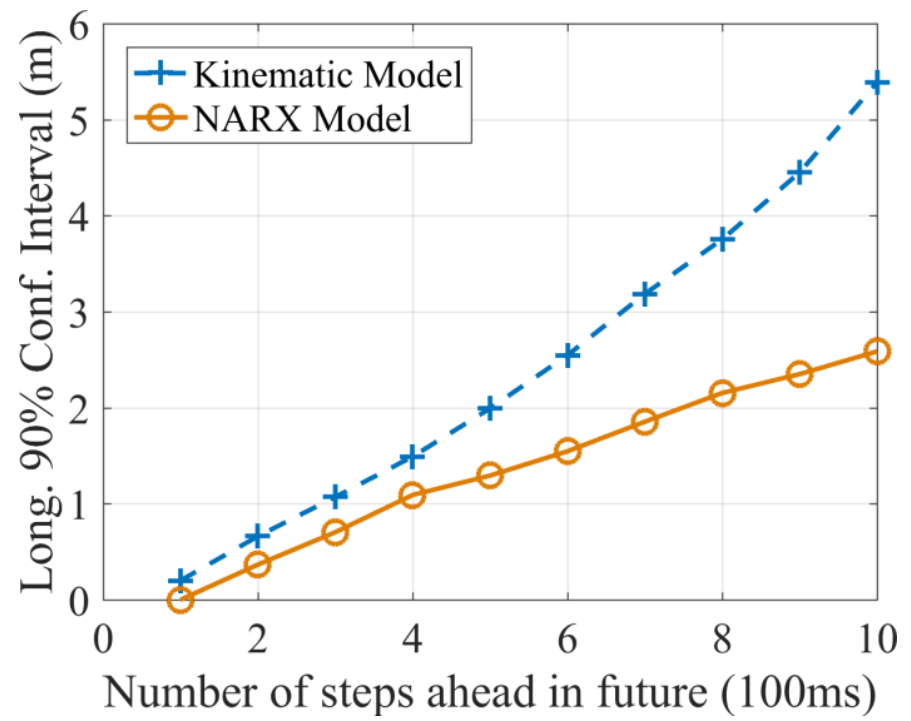

Figure 5-6- Comparison of 90-percentile conf. interval of Longitudinal position prediction of the Kinematic and RNN models for different prediction steps

Finally, figure (a)-(g) shows the snapshots of the performance of the Stochastic-MPC for CACC during a lane change maneuver. The predicted trajectory of the cut-in vehicle for one second is shown with purple circles surrounded by a confidence rectangle for each prediction. The bad-set of the host vehicle is depicted as a green rectangle. The blue dots are showing the actual cut-in maneuver and the red dots are showing the long-term prediction results, which is the prediction beyond the one-second short-term window.

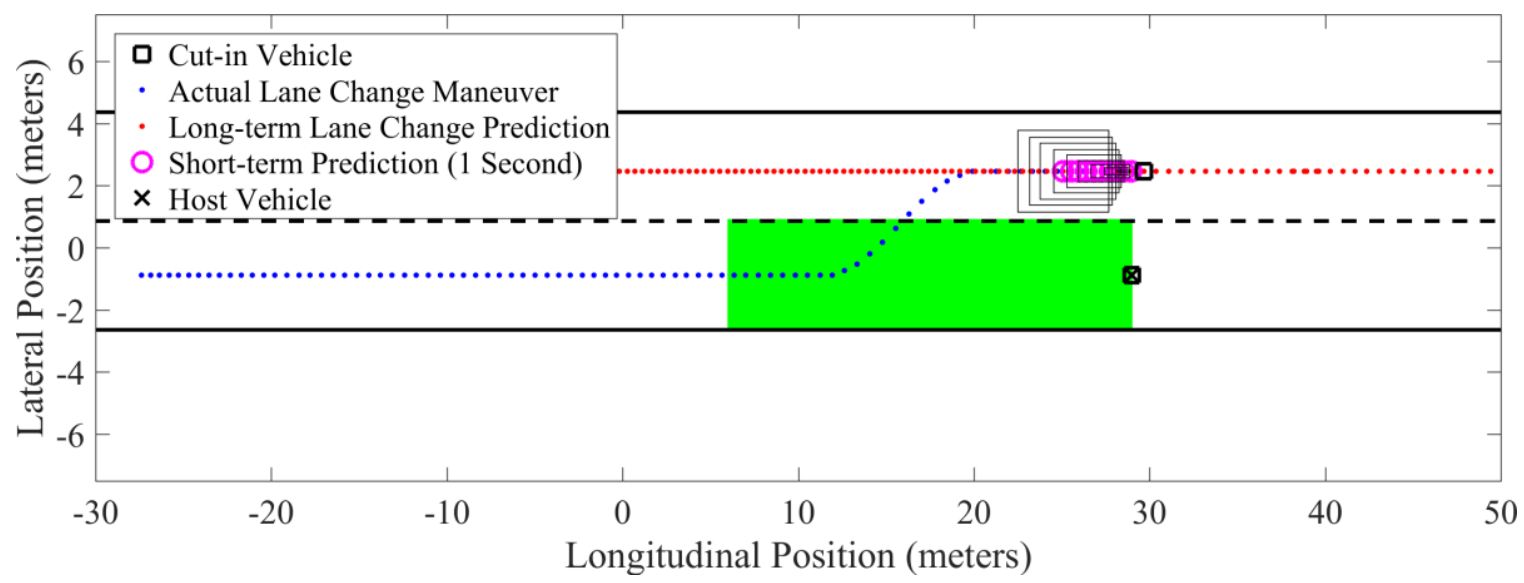

(a) Cut-in vehicle prepares for lane change 


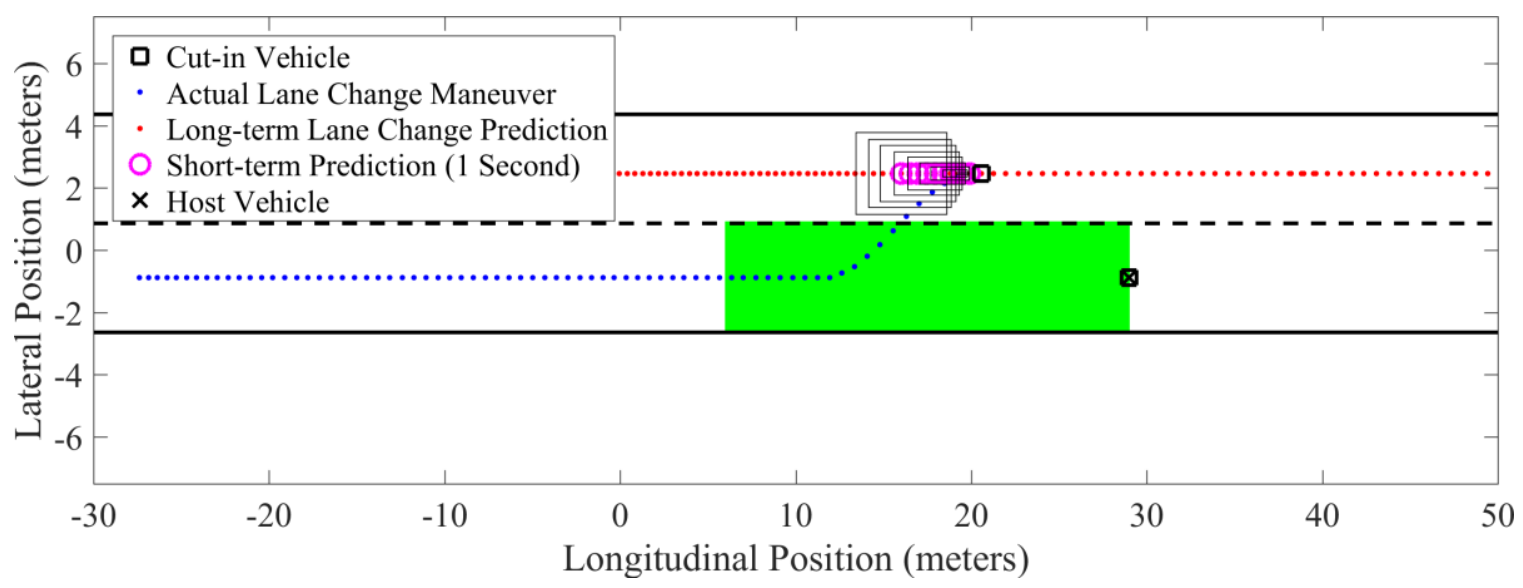

(b) The transition phase of lane change begins

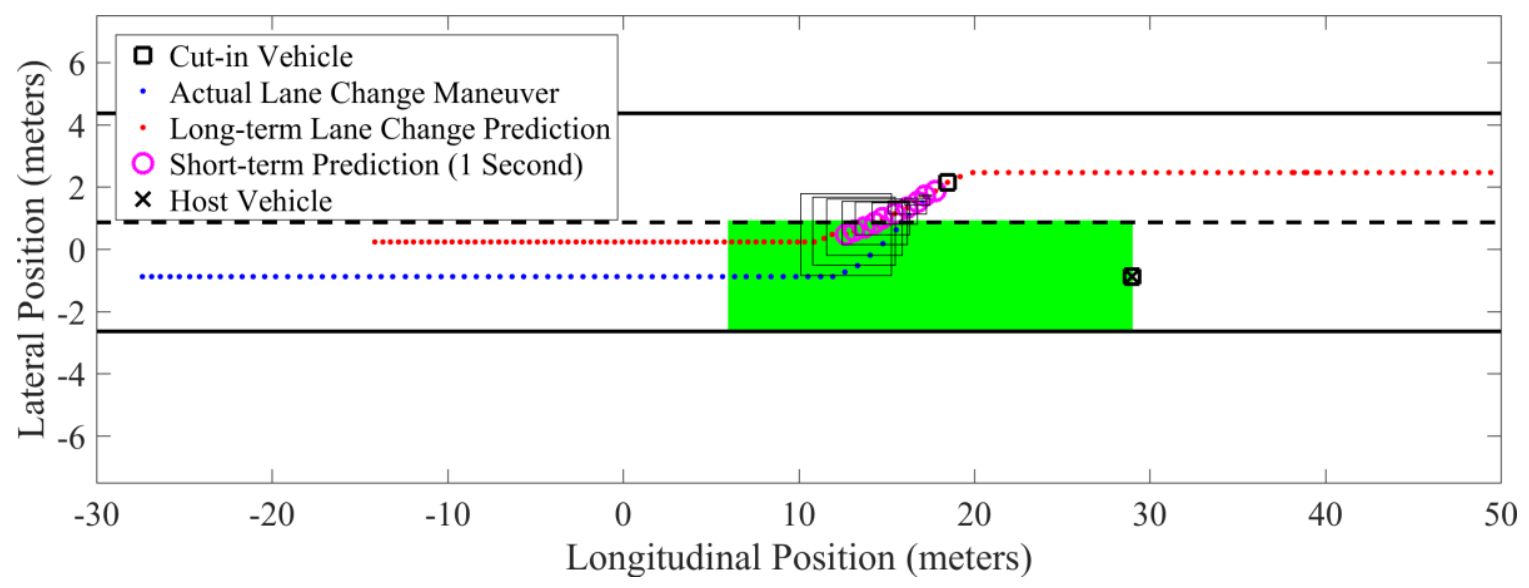

(c) Few steps after commence of lateral movement

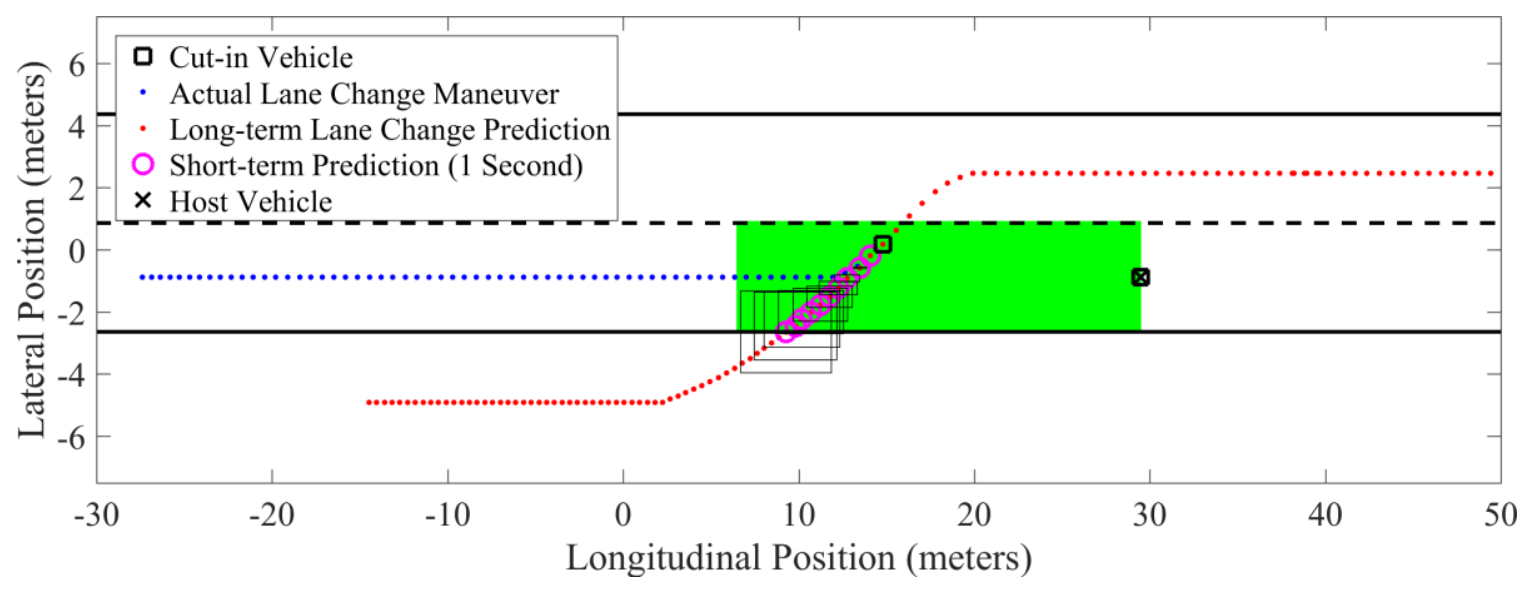

(d) Half-way through lane change, the cut-in vehicles crossed the middle line 


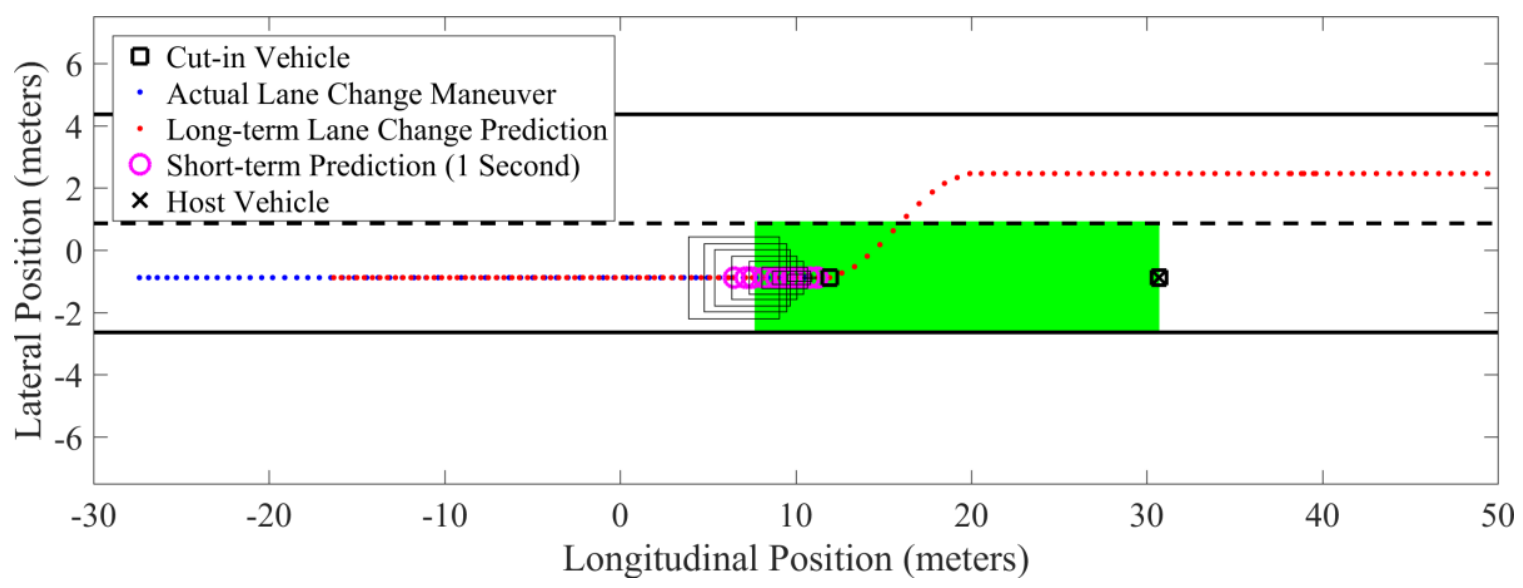

(e) End of transition phase

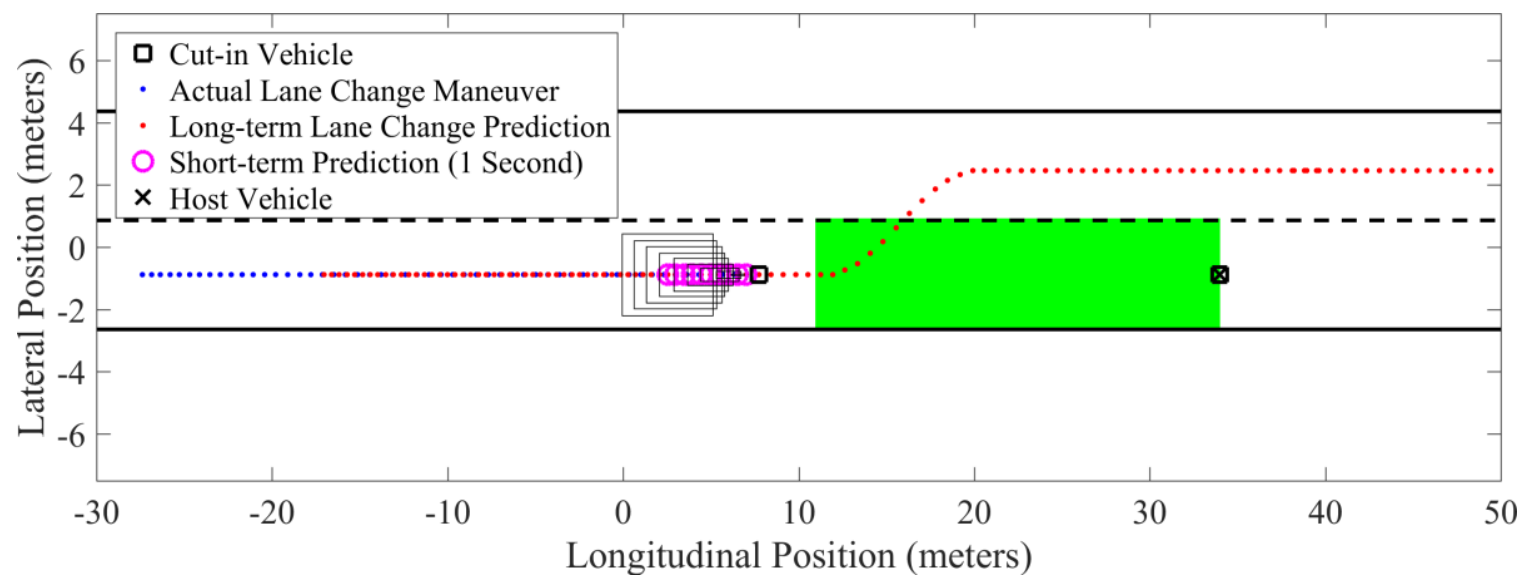

(f) Completion phase for cut-in and adjustment of separation distance by Stochastic-MPC

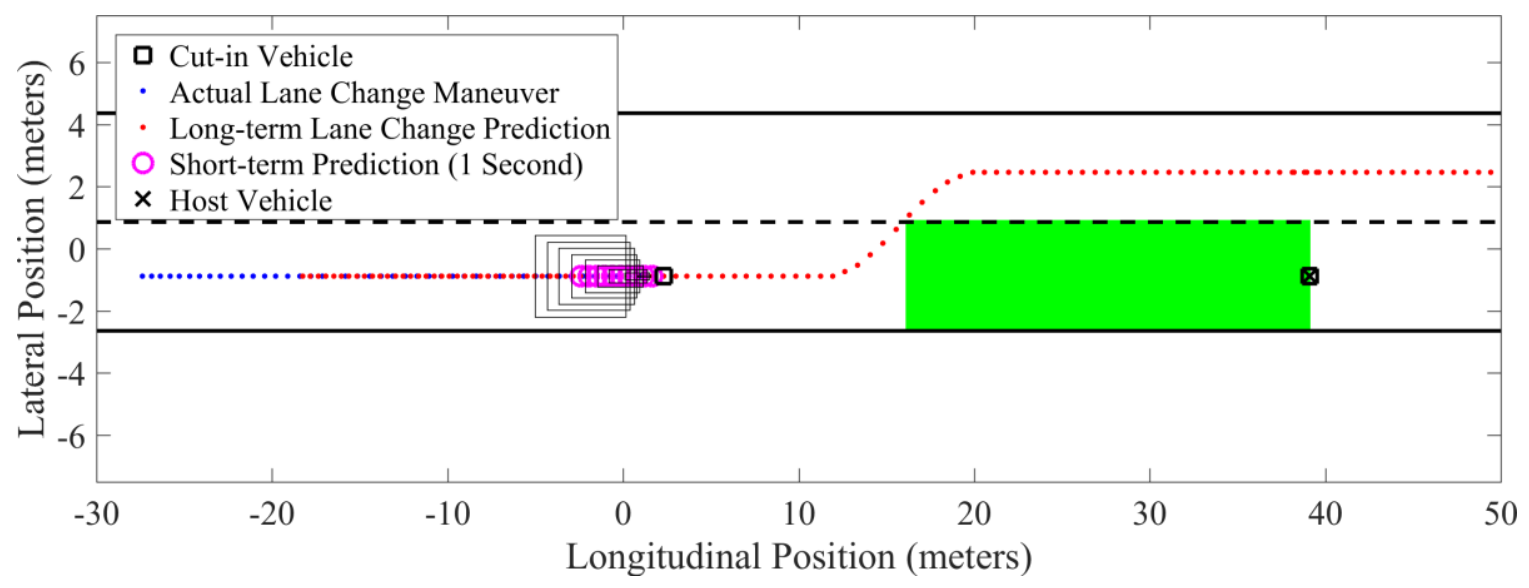

(g) Host vehicle following the cut-in vehicle as the new leading vehicle

Figure 5-7- (a)-(g) Lane change prediction and MPC for CACC 


\section{Chapter 6 Conclusion and Future Works}

In this dissertation, we focused on the enhancement of situational awareness and its applications for Connected and Automated Vehicle systems (CAVs). We first proposed a new modeling scheme for large-scale and small-scale components of the vehicular communication channel in dense vehicular networks. Subsequently, we designed an innovative method for a joint modeling of the situational awareness and communication components of CAVs in a single framework to achieve real-time situational awareness beyond line-of-sight (LOS). Based on these two models, we proposed a novel network-aware broadcast protocol for fast broadcasting of information over multiple hops to extend the range of situational awareness. Afterward, we extended our work to an end-to-end Vehicle-to-Pedestrian (V2P) framework to provide situational awareness and hazard detection for vulnerable road users. Finally, we designed a learning-based driver behavior model and integrate it into the situational awareness component of a platooning system. Consequently, higher accuracy of situational awareness and overall system performance are achieved by exchange of more useful information.

We investigated the effect of vehicular communication on situational awareness in Chapter 2. We first proposed an approach to remove the effect of fading on the deterministic part of the largescale model. The two-ray model was then utilized for path-loss characterization and its parameters were derived from the empirical data based on our proposed method. Furthermore, we studied the usage of $\alpha-\mu$ distribution to model the fading behavior of vehicular networks for the first time and verified its veracity by the K-S goodness-of-fit test. Based on the presented results of this section, $\alpha-\mu$ distribution is observed to be a promising approach to model the fading behavior. Therefore, we are planning to extend the proposed approach for other dense vehicular environments such as intersections and tunnels. We then studied the real-time situational awareness subsystem of NCAS consists of two major components of estimation and communication. These two components have been modeled in a single framework. A PTA model was presented to model the communication component, and a hybrid automaton was proposed to model the entire system. This framework can be extended to study other estimation processes. The remaining parts of the model would remain intact. A rate control scheme can also be modeled as a timed transition out of this state based on network feedback. In addition, the communication part can potentially be enhanced to allow power or range variations due to the application of adaptive power control methods. 
Chapter 3 described a network-aware double-layer distance-dependent protocol to ensure low latency and scalability over multiple hops. The first distance-dependent layer affects the rebroadcast decisions inside a hop and had two parts. The first part was a message size control scheme to utilize a small portion of channel load. The second part was a mix probabilistic rebroadcast method to give a higher probability of message rebroadcast to vehicles that are not only farther from the last sender but also in better communication condition, to guarantee that the forwarding within each hop is as fast as possible. In the second layer, the rate of information forwarding is decreased based on distance from the information source to preserve channel load. The effect was the rapid dissemination of information with a diminishing rate of forwarding. The worst-case boundary of the forwarding delay is still one order of magnitude higher than flooding with any of the techniques such as counter based and probability-based that are likely to have the same latency at the cost of much higher overload. Thus, further improvements might be possible through other distance-dependent methods for the second layer. We also aim to present the performance evaluation of the proposed method by generalizing the road scenarios to intersections and other movement topologies in which density changes quickly. Finally, in our future works, we would like to analyze the impact of our algorithm on the quality of real-time navigation systems and its effect on travel time calculation and rerouting algorithms.

Chapter 4 presented design, implementation, testing, and evaluation of our proposed vehicle-topedestrian safety system. Particular characteristics of VRU movement dynamics, response times, and most fatal crash scenarios were considered in this framework to extend situational awareness and hazard detection capabilities. Furthermore, the proposed framework was designed to satisfy the scalability requirements of cooperative vehicle-VRU networks. Thus, power consumption and channel congestion mechanisms were described based on the particular needs of such networks. The reported practical tests and analyses indicated that our design is a promising VRU crash avoidance and mitigation solution. Our future work includes large-scale trials and empirical parameter optimization of proposed congestion control and power consumption control mechanisms. Although the presented system design seems to provide a cornerstone for VRUs to talk to vehicles for safety, the realization of V2P safety systems is still facing many challenges. Further improvements and investigations in many aspects, such as network congestion, power consumption, localization, security, and availability of DSRC radio in a smartphone, are still required.

In Chapter 5, we designed a learning-based driver behavior model and integrated it into the estimation component of our situational awareness system. We specifically concentrated on cutin maneuvers for CACC platoons, as a vehicle in a stable CACC platoon has to hard brake when another vehicle changes its lane into the platoon just in front of it. Therefore, time series were used to model the trajectory of the vehicle. Nonlinear autoregressive (NAR), Nonlinear autoregressive exogenous (NARX), and recurrent neural networks (RNN) were trained and used for lane change prediction. We then used the outcome of the ANNs as an input to a Stochastic-MPC. Three main goals are achieved with this controller. The controller minimizes the spacing error between the host vehicle and the vehicle in front of it while keeping the velocity as close as possible to the velocity of leading vehicle. Simultaneously, since it knows the probability of lane change from the vehicle in the adjacent lane, it responds appropriately to a cut-in maneuver to avoid harsh braking. This probabilistic approach contributes to the stability of CACC and platooning. The designed controller gives a safe cut-in maneuver opportunity to the cut-in vehicle by increasing the distance with its leading vehicle. In our future works, since the controller and ANNs were designed for lane 
change maneuvers on straight roads, we would like to design and analyze the impact of our method on lane changes in more complex environments such as curvy road or exit ramps. 


\section{References}

[1] J. B. Kenney. Dedicated short-range communications (DSRC) standards in the united states. Proceedings of the IEEE 99(7), pp. 1162-1182. 2011.

[2] Cheng-Xiang Wang, Xiang Cheng and D. I. Laurenson. Vehicle-to-vehicle channel modeling and measurements: Recent advances and future challenges. Communications Magazine, IEEE 47(11), pp. 96-103. 2009.

[3] A. F. Molisch, F. Tufvesson, J. Karedal and C. F. Mecklenbrauker. A survey on vehicle-to-vehicle propagation channels. Wireless Communications, IEEE 16(6), pp. 12-22. 2009.

[4] W. Viriyasitavat, M. Boban, Hsin-Mu Tsai and A. Vasilakos. Vehicular communications: Survey and challenges of channel and propagation models. Vehicular Technology Magazine, IEEE 10(2), pp. 55-66. 2015.

[5] Lin Cheng, B. Henty, Fan Bai and D. D. Stancil. Doppler spread and coherence time of rural and highway vehicleto-vehicle channels at $5.9 \mathrm{GHz}$. Presented at Global Telecommunications Conference, 2008. IEEE GLOBECOM 2008. IEEE. 2008.

[6] J. Kunisch and J. Pamp. Wideband car-to-car radio channel measurements and model at $5.9 \mathrm{GHz}$. Presented at Vehicular Technology Conference, 2008. VTC 2008-Fall. IEEE 68th. 2008.

[7] J. Karedal, N. Czink, A. Paier, F. Tufvesson and A. F. Molisch. Path loss modeling for vehicle-to-vehicle communications. Vehicular Technology, IEEE Transactions On 60(1), pp. 323-328. 2011.

[8] A. Paier, J. Karedal, N. Czink, C. Dumard, T. Zemen, F. Tufvesson, A. Molisch and C. Mecklenbräuker. Characterization of vehicle-to-vehicle radio channels from measurements at $5.2 \mathrm{GHz}$. Wireless Personal Communications 50(1), pp. 19-32. 2009.

[9] A. Paier, J. Karedal, N. Czink, H. Hofstetter, C. Dumard, T. Zemen, F. Tufvesson, A. F. Molisch and C. F. Mecklenbrauker. Car-to-car radio channel measurements at $5 \mathrm{GHz}$ : Pathloss, power-delay profile, and delaydoppler spectrum. Presented at Wireless Communication Systems, 2007. ISWCS 2007. 4th International Symposium On. 2007.

[10] A. Paier, J. Karedal, N. Czink, H. Hofstetter, C. Dumard, T. Zemen, F. Tufvesson, C. F. Mecklenbrauker and A. F. Molisch. First results from car-to-car and car-to-infrastructure radio channel measurements at 5.2GHZ. Presented at Personal, Indoor and Mobile Radio Communications, 2007. PIMRC 2007. IEEE 18th International Symposium On. 2007.

[11] J. Maurer, T. Fugen and W. Wiesbeck. Narrow-band measurement and analysis of the inter-vehicle transmission channel at 5.2 GHz. Presented at Vehicular Technology Conference, 2002. VTC Spring 2002. IEEE 55th. 2002.

[12] G. Acosta-marum and M. Ingram Ann, "Doubly selective vehicle-to-vehicle channel measurements and modeling at $5.9 \mathrm{GHz}$, in Proc. Int. Symp. Wireless Personal Multimedia Commun, 2006.

[13] G. Acosta, K. Tokuda and M. A. Ingram. Measured joint doppler-delay power profiles for vehicle-to-vehicle communications at $2.4 \mathrm{GHz}$. Presented at Global Telecommunications Conference, 2004. GLOBECOM '04. IEEE. 2004.

[14] Jijun Yin, G. Holland, T. ElBatt, Fan Bai and H. Krishnan. DSRC channel fading analysis from empirical measurement. Presented at Communications and Networking in China, 2006. ChinaCom '06. First International Conference On. 2006.

[15] M. Boban, W. Viriyasitavat and O. K. Tonguz, "Modeling vehicle-to-vehicle line of sight channels and its impact on application-layer performance," in Proceeding of the Tenth ACM International Workshop on Vehicular InterNetworking, Systems, and Applications, New York, NY, USA, 2013pp. 91-94.

[16] M. Boban, R. Meireles, J. Barros, O. Tonguz and P. Steenkiste. Exploiting the height of vehicles in vehicular communication. Presented at Vehicular Networking Conference (VNC), 2011 IEEE. 2011.

[17] M. Boban, T. T. V. Vinhoza, M. Ferreira, J. Barros and O. K. Tonguz. Impact of vehicles as obstacles in vehicular ad hoc networks. Selected Areas in Communications, IEEE Journal On 29(1), pp. 15-28. 2011.

[18] R. Meireles, M. Boban, P. Steenkiste, O. Tonguz and J. Barros. Experimental study on the impact of vehicular obstructions in VANETs. Presented at Vehicular Networking Conference (VNC), 2010 IEEE. 2010.

[19] Lin Cheng, B. E. Henty, D. D. Stancil, Fan Bai and P. Mudalige. Mobile vehicle-to-vehicle narrow-band channel measurement and characterization of the $5.9 \mathrm{GHz}$ dedicated short range communication (DSRC) frequency band. Selected Areas in Communications, IEEE Journal On 25(8), pp. 1501-1516. 2007.

[20] Lin Cheng, B. E. Henty, Fan Bai and D. D. Stancil. Highway and rural propagation channel modeling for vehicleto-vehicle communications at $5.9 \mathrm{GHz}$. Presented at Antennas and Propagation Society International Symposium, 2008. AP-S 2008. IEEE. 2008. 
[21] G. P. Grau, D. Pusceddu, S. Rea, O. Brickley, M. Koubek and D. Pesch. Vehicle-2-vehicle communication channel evaluation using the CVIS platform. Presented at Communication Systems Networks and Digital Signal Processing (CSNDSP), 2010 7th International Symposium On. 2010.

[22] V. Taliwal, D. Jiang, H. Mangold, C. Chen and R. Sengupta, "Empirical determination of channel characteristics for DSRC vehicle-to-vehicle communication," in Proceedings of the 1st ACM International Workshop on Vehicular Ad Hoc Networks, Philadelphia, PA, USA, 2004pp. 88.

[23] T. Islam, Yongchang Hu, E. Onur, B. Boltjes and J. F. C. M. de Jongh. Realistic simulation of IEEE 802.11p channel in mobile vehicle to vehicle communication. Presented at Microwave Techniques (COMITE), 20131 Conference On. 2013.

[24]F. Ahmed-Zaid, F. Bai, S. Bai, C. Basnayake, B. Bellur, S. Brovold, G. Brown, L. Caminiti, D. Cunningham, H. Elzein, K. Hong, J. Ivan, D. Jiang, J. Kenney, H. Krishnan, J. Lovell, M. Maile, D. Masselink, E. McGlohon, P. Mudalige, Z. Popovic, V. Rai, J. Stinnett, L. Tellis, K. Tirey and S. VanSickle, "Vehicle safety communications - applications (VSC-A) final report," Tech. Rep. DOT HS 811 492A, September 2011.

[25] The CAMP Vehicle Safety Communications Consortium consisting of BMW, DaimlerChrysler, Ford, GM, Nissan, Toyota, and VW., "Vehicle safety communications project task 3 final report - identify intelligent vehicle safety applications enabled by DSRC," Tech. Rep. DOT HS 809 859, March 2005.

[26] S. Rezaei, R. Sengupta, H. Krishnan, X. Guan and R. Bhatia. Tracking the position of neighboring vehicles using wireless communications. Transportation Research Part C: Emerging Technologies 18(3), pp. 335-350. 2010.

[27]C. Huang, Y. P. Fallah, R. Sengupta and H. Krishnan. Adaptive intervehicle communication control for cooperative safety systems. IEEE Network, 24(1), pp. 6-13. 2010.

[28] Q. Chen, D. Jiang, T. Tielert and L. Delgrossi. Mathematical modeling of channel load in vehicle safety communications. Presented at Vehicular Technology Conference (VTC Fall), 2011 IEEE. 2011.

[29] Y. P. Fallah, C. Huang, R. Sengupta and H. Krishnan. Design of cooperative vehicle safety systems based on tight coupling of communication, computing and physical vehicle dynamics. Presented at Proceedings of the 1st ACM/IEEE International Conference on Cyber-Physical Systems. 2010.

[30] Y. P. Fallah and R. Sengupta. A cyber-physical systems approach to the design of vehicle safety networks. Presented at 2012 32nd International Conference on Distributed Computing Systems Workshops. 2012.

[31] Y. P. Fallah, C. L. Huang, R. Sengupta and H. Krishnan. Analysis of information dissemination in vehicular adhoc networks with application to cooperative vehicle safety systems. IEEE Transactions on Vehicular Technology 60(1), pp. 233-247. 2011.

[32] Y. P. Fallah, C. Huang, R. Sengupta and H. Krishnan. Congestion control based on channel occupancy in vehicular broadcast networks. Presented at IEEE 72nd Vehicular Technology Conference Fall, 2010.

[33] E. Moradi-Pari, A. Tahmasbi-Sarvestani and Y. P. Fallah. Modeling communication and estimation processes of automated crash avoidance systems. Presented at Systems Conference (SysCon), 2013 IEEE International. 2013.

[34] C. G. Cassandras and S. Lafortune, Introduction to Discrete Event Systems. Springer US, 2008.

[35] R. Alur and D. L. Dill. A theory of timed automata. Theor. Comput. Sci. 126(2), pp. 183-235. 1994.

[36] G. Rodriguez-Navas and J. Proenza. Using timed automata for modeling distributed systems with clocks: Challenges and solutions. IEEE Transactions on Software Engineering 39(6), pp. 857-868. 2013.

[37] M. Kwiatkowska, G. Norman and J. Sproston. Probabilistic Model Checking of the IEEE 802.11 Wireless Local Area Network Protocol 2002.

[38] A. Heindl and R. German. Performance modeling of IEEE 802.11 wireless LANs with stochastic petri nets. Performance Evaluation 44(1), pp. 139-164. 2001.

[39] M. Fruth. Probabilistic model checking of contention resolution in the IEEE 802.15. 4 low-rate wireless personal area network protocol. Presented at Leveraging Applications of Formal Methods, Verification and Validation, 2006. ISoLA 2006. Second International Symposium On. 2006.

[40] M. Kwiatkowska, G. Norman, D. Parker and J. Sproston. Performance analysis of probabilistic timed automata using digital clocks. Formal Methods Syst. Des. 29(1), pp. 33-78. 2006.

[41] M. M. I. Taha and Y. M. Y. Hasan. VANET-DSRC protocol for reliable broadcasting of life safety messages. Presented at IEEE International Symposium on Signal Processing and Information Technology, 2007.

[42] K. Dar, M. Bakhouya, J. Gaber, M. Wack and P. Lorenz. Wireless communication technologies for ITS applications [topics in automotive networking]. IEEE Communications Magazine, 48(5), pp. 156-162. 2010.

[43] E. Schoch, F. Kargl, M. Weber and T. Leinmuller. Communication patterns in VANETs. IEEE Communications Magazine, 46(11), pp. 119-125. 2008.

[44] W. K. Wolterink, G. J. Heijenk and G. Karagiannis. Information dissemination in VANETS by piggybacking on beacons - an analysis of the impact of network parameters. Presented at IEEE Vehicular Networking Conference, 2011. 
[45] L. Yang, J. Guo and Y. Wu. Piggyback cooperative repetition for reliable broadcasting of safety messages in VANETs. Presented at 6th IEEE Consumer Communications and Networking Conference, 2009.

[46] S. A. Ahmed, S. H. Ariffin, N. Fisal, S. Syed-Yusof and N. Latif. Survey on broadcasting in VANETs. Research Journal of Applied Sciences, Engineering and Technology 7(18), pp. 3733 - 3739. 2014.

[47] S. Cha. A survey of broadcast protocols for vehicular ad-hoc networks. Smart Computing Review 4(4), pp. 246255. 2014.

[48] M. Chaqfeh, A. Lakas and I. Jawhar. A survey on data dissemination in vehicular ad hoc networks. Vehicular Communications 1(4), pp. 214-225. 2014.

[49] O. Tonguz, N. Wisitpongphan, F. Bai, P. Mudalige and V. Sadekar. Broadcasting in VANET. Presented at Mobile Networking for Vehicular Environments. 2007.

[50] L. Briesemeister and G. Hommel. Role-based multicast in highly mobile but sparsely connected ad hoc networks. Presented at Proceedings of the 1st ACM International Symposium on Mobile Ad Hoc Networking \& Computing. 2000.

[51]Z. D. Chen, H. Kung and D. Vlah. Ad hoc relay wireless networks over moving vehicles on highways. Presented at Proceedings of the 2nd ACM International Symposium on Mobile Ad Hoc Networking \& Computing. 2001.

[52] J. Zhao and G. Cao. VADD: Vehicle-assisted data delivery in vehicular ad hoc networks. Presented at Proceeding of 25th IEEE International Conference on Computer Communications, 2006.

[53] O. K. Tonguz, N. Wisitpongphan and Fan Bai. DV-CAST: A distributed vehicular broadcast protocol for vehicular ad hoc networks. Wireless Communications, IEEE 17(2), pp. 47-57. 2010.

[54] S. Yu and G. Cho. A selective flooding method for propagating emergency messages in vehicle safety communications. Presented at International Conference on Hybrid Information Technology, 2006.

[55] A. Amoroso, M. Ciaschini and M. Roccetti. The farther relay and oracle for VANET. preliminary results. Presented at Proceedings of the 4th Annual International Conference on Wireless Internet. 2008.

[56] M. Sun, W. Feng, T. Lai, K. Yamada, H. Okada and K. Fujimura. GPS-based message broadcasting for intervehicle communication. Presented at Proceedings of International Conference on Parallel Processing, 2000.

[57] A. M. Vegni, A. Stramacci and E. Natalizio. SRB: A selective reliable broadcast protocol for safety applications in VANETs. Presented at International Conference on Selected Topics in Mobile and Wireless Networking, 2012.

[58] G. Korkmaz, E. Ekici, F. Özgüner and Ü Özgüner. Urban multi-hop broadcast protocol for inter-vehicle communication systems. Presented at Proceedings of the 1st ACM International Workshop on Vehicular Ad Hoc Networks. 2004.

[59] G. Korkmaz, E. Ekici and F. Ozguner. An efficient fully ad-hoc multi-hop broadcast protocol for inter-vehicular communication systems. Presented at IEEE International Conference on Communications, 2006.

[60] A. Amoroso, G. Marfia and M. Roccetti. Going realistic and optimal: A distributed multi-hop broadcast algorithm for vehicular safety. Computer Networks 55(10), pp. 2504-2519. 2011.

[61] A. Benaidja, S. Moussaoui and F. Naït-Abdesselam. An optimal broadcast of warning messages in vehicular ad hoc networks. International Journal of Computer and Information Technology 02(05), 2013.

[62] M. N. Mariyasagayam, T. Osafune and M. Lenardi. Enhanced multi-hop vehicular broadcast (MHVB) for active safety applications. Presented at 7th International Conference on ITS Telecommunications, 2007.

[63] Y. Peksen and T. Acarman. Relay of multi-hop safety message based on beaconing in VANET. Presented at IEEE International Conference on Vehicular Electronics and Safety, 2012.

[64] N. Wisitpongphan, O. K. Tonguz, J. S. Parikh, P. Mudalige, F. Bai and V. Sadekar. Broadcast storm mitigation techniques in vehicular ad hoc networks. IEEE Wireless Communications, 14(6), pp. 84-94. 2007.

[65] Y. Sung and M. Lee. Light-weight reliable broadcast message delivery for vehicular ad-hoc networks. Presented at IEEE 75th Vehicular Technology Conference Spring, 2012.

[66] H. ALshaer and E. Horlait. An optimized adaptive broadcast scheme for inter-vehicle communication. Presented at IEEE 61st Vehicular Technology Conference, 2005.

[67]H. Jiang, H. Guo and L. Chen. Reliable and efficient alarm message routing in VANET. Presented at 28th International Conference on Distributed Computing Systems Workshops, 2008.

[68] V. Drabkin, R. Friedman, G. Kliot and M. Segal. RAPID: Reliable probabilistic dissemination in wireless ad-hoc networks. Presented at 26th IEEE International Symposium on Reliable Distributed Systems, 2007.

[69] I. A. Khan, A. Javaid and H. L. Qian. Distance-based dynamically adjusted probabilistic forwarding for wireless mobile ad hoc networks. Presented at 5th IFIP International Conference on Wireless and Optical Communications Networks, 2008.

[70] NHTSA, "National pedestrian crash report," Tech. Rep. DOT HS 810968, June 2008.

[71] C. Zegeer, D. Nabors, D. Gelinne, N. Lefler and M. Bushell, "Pedestrian safety strategic plan: Recommendations for research and product development," FHWA, Tech. Rep. FHWA-10-035, 2010. 
[72] G. Gate, A. Breheret and F. Nashashibi. Centralized fusion for fast people detection in dense environment. Presented at Robotics and Automation, 2009. ICRA '09. IEEE International Conference On. 2009.

[73]T. Gandhi and M. M. Trivedi. Pedestrian protection systems: Issues, survey, and challenges. Intelligent Transportation Systems, IEEE Transactions On 8(3), pp. 413-430. 2007.

[74] M. G. Carpenter, M. T. Moury, J. R. Skvarce, M. Struck, T. D. Zwicky and S. M. Kiger, "Objective tests for forward looking pedestrian crash avoidance/mitigation systems, final report," Tech. Rep. DOT HS 812 040, June 2014.

[75] M. Liebner, F. Klanner and C. Stiller. Active safety for vulnerable road users based on smartphone position data. Presented at Intelligent Vehicles Symposium (IV), 2013 IEEE. 2013.

[76] A. Fackelmeier, C. Morhart and E. Biebl. "Dual frequency methods for identifying hidden targets in road traffic," in Advanced Microsystems for Automotive Applications 2008Anonymous 2008, Available: http://dx.doi.org/10.1007/978-3-540-77980-3 2. DOI: 10.1007/978-3-540-77980-3_2.

[77] R. H. Rasshofer, D. Schwarz, E. Biebl, C. Morhart, O. Scherf and S. Zecha, "Pedestrian protection systems using cooperative sensor technology," in Advanced Microsystems for Automotive Applications 2007Anonymous Springer Berlin Heidelberg, 2007-01-01, pp. 135-145.

[78] C. Sugimoto, Y. Nakamura and T. Hashimoto. Development of pedestrian-to-vehicle communication system prototype for pedestrian safety using both wide-area and direct communication. Presented at Advanced Information Networking and Applications, 2008. AINA 2008. 22nd International Conference On. 2008.

[79] C. Sugimoto, Y. Nakamura and T. Hashimoto. Prototype of pedestrian-to-vehicle communication system for the prevention of pedestrian accidents using both $3 \mathrm{G}$ wireless and WLAN communication. Presented at Wireless Pervasive Computing, 2008. ISWPC 2008. 3rd International Symposium On. 2008.

[80] A. Flach and K. David. A physical analysis of an accident scenario between cars and pedestrians. Presented at Vehicular Technology Conference Fall (VTC 2009-Fall), 2009 IEEE 70th. 2009.

[81] K. David and A. Flach. CAR-2-X and pedestrian safety. Vehicular Technology Magazine, IEEE 5(1), pp. 70-76. 2010.

[82] M. Yanagisawa, E. Swanson and W. G. Najm, "Target crashes and safety benefits estimation methodology for pedestrian crash avoidance/mitigation systems," Tech. Rep. DOT HS 811 998, April 2014.

[83] GM news. (July 2012). GM Developing Wireless Pedestrian Detection Technology. Available: http://media.gm.com/media/us/en/gm/news.detail.html/content/Pages/news/us/en/2012/Jul/0726_pedestrian.htm 1.

[84] J. J. Anaya, P. Merdrignac, O. Shagdar, F. Nashashibi and J. E. Naranjo. Vehicle to pedestrian communications for protection of vulnerable road users. Presented at Intelligent Vehicles Symposium Proceedings, 2014 IEEE. 2014.

[85]F. Bai, D. D. Stancil and H. Krishnan, "Toward understanding characteristics of dedicated short range communications (DSRC) from a perspective of vehicular network engineers," in Proceedings of the Sixteenth Annual International Conference on Mobile Computing and Networking (MobiCom '10), Chicago, Illinois, USA, 2010.

[86] SAE International, "Dedicated Short Range Communications (DSRC) Message Set Dictionary," SAE Standard J2735_201601, Jan. 2016.

[87] NHTSA, "Vehicle-toVehicle communications: Readiness of V2V technology for application," Tech. Rep. DOT HS 812 014, August 2014.

[88] X. Wu, R. Miucic, S. Yang, S. Al-Stouhi, J. Misener, S. Bai and W. h. Chan. Cars talk to phones: A DSRC based vehicle-pedestrian safety system. Presented at Vehicular Technology Conference (VTC Fall), 2014 IEEE 80th. 2014.

[89] A. Tahmasbi-Sarvestani, H. Kazemi, Y. P. Fallah, M. Naserian and et al., "System architecture for cooperative vehicle-pedestrian safety applications using DSRC communication," in SAE Technical Paper 2015-01-0290, 2015.

[90] M. A. Abid, O. Chakroun and S. Cherkaoui. Pedestrian collision avoidance in vehicular networks. Presented at Communications (ICC), 2013 IEEE International Conference On. 2013.

[91] S. Bonnin, F. Kummert and J. Schmüdderich. A generic concept of a system for predicting driving behaviors. Presented at 2012 15th International IEEE Conference on Intelligent Transportation Systems. 2012.

[92] H. M. Mandalia and M. D. D. Salvucci. Using support vector machines for lane-change detection. Presented at Proceedings of the Human Factors and Ergonomics Society Annual Meeting. 2005.

[93]D. Kasper, G. Weidl, T. Dang, G. Breuel, A. Tamke, A. Wedel and W. Rosenstiel. Object-oriented bayesian networks for detection of lane change maneuvers. IEEE Intelligent Transportation Systems Magazine 4(3), pp. 19-31. 2012. 
[94]T. Gindele, S. Brechtel and R. Dillmann. A probabilistic model for estimating driver behaviors and vehicle trajectories in traffic environments. Presented at Intelligent Transportation Systems (ITSC), 2010 13th International IEEE Conference On. 2010.

[95] A. Pentland and A. Liu. Modeling and prediction of human behavior. Neural Comput. 11(1), pp. 229-242. 1999.

[96] N. Kuge, T. Yamamura, O. Shimoyama and A. Liu. A driver behavior recognition method based on a driver model framework. 2000.

[97] H. Berndt, J. Emmert and K. Dietmayer. Continuous driver intention recognition with hidden markov models. Presented at 2008 11th International IEEE Conference on Intelligent Transportation Systems. 2008.

[98] N. Oliver and A. P. Pentland. Graphical models for driver behavior recognition in a smartcar. Presented at Intelligent Vehicles Symposium, 2000. IV 2000. Proceedings of the IEEE. 2000.

[99] M. Mori, C. Miyajima, T. Hirayama, N. Kitaoka and K. Takeda. Integrated modeling of driver gaze and vehicle operation behavior to estimate risk level during lane changes. Presented at 16th International IEEE Conference on Intelligent Transportation Systems (ITSC 2013). 2013.

[100] B. Morris, A. Doshi and M. Trivedi. Lane change intent prediction for driver assistance: On-road design and evaluation. Presented at Intelligent Vehicles Symposium (IV), 2011 IEEE. 2011.

[101] D. Mitrovic. Reliable method for driving events recognition. IEEE Transactions on Intelligent Transportation Systems 6(2), pp. 198-205. 2005.

[102] P. Liu, A. Kurt and Ü özgüner. Trajectory prediction of a lane changing vehicle based on driver behavior estimation and classification. Presented at 17th International IEEE Conference on Intelligent Transportation Systems (ITSC). 2014.

[103] P. Liu and Ü Özgüner. Predictive control of a vehicle convoy considering lane change behavior of the preceding vehicle. Presented at 2015 American Control Conference (ACC). 2015.

[104] R. S. Tomar, S. Verma and G. S. Tomar. Prediction of lane change trajectories through neural network. Presented at Computational Intelligence and Communication Networks (CICN), 2010 International Conference On. 2010.

[105] V. Milanés and S. E. Shladover. Handling cut-in vehicles in strings of cooperative adaptive cruise control vehicles. Journal of Intelligent Transportation Systems 20(2), pp. 178-191. 2016.

[106] E. Moradi-Pari, A. Tahmasbi-Sarvestani and Y. P. Fallah. A hybrid systems approach to modeling real-time situation-awareness component of networked crash avoidance systems. IEEE Systems Journal, PP(99), pp. 1-10. 2014.

[107] A. Tahmasbi-Sarvestani, Y. P. Fallah and V. Kulathumani. Network-aware double-layer distance-dependent broadcast protocol for VANETs. Vehicular Technology, IEEE Transactions On 64(12), pp. 5536-5546. 2015.

[108] W. Viriyasitavat and M. Boban, "Channel models for vehicular communications," in Vehicular Ad Hoc Networks; Standards, Solutions, and Research, First ed.Anonymous Springer, 2015, pp. 335-363.

[109] D. W. Matolak and Qiong Wu. Vehicle-to-vehicle channels: Are we done yet? Presented at GLOBECOM Workshops, 2009 IEEE. 2009.

[110] D. W. Matolak. Channel modeling for vehicle-to-vehicle communications. Communications Magazine, IEEE 46(5), pp. 76-83. 2008.

[111] A. F. Molisch, F. Tufvesson, J. Karedal and C. Mecklenbrauker. Propagation aspects of vehicle-to-vehicle communications - an overview. Presented at Radio and Wireless Symposium, 2009. RWS '09. IEEE. 2009.

[112] S. M. O. Gani, A. Tahmasbi-Sarvestani, M. Fanaei and Y. P. Fallah, "High fidelity DSRC receiver model for ns-3 simulation using large-scale field data," in Ieee Wcnc 2016, Doha, Qatar, 2016.

[113] M. Fanaei, A. Tahmasbi-Sarvestani, Y. P. Fallah, G. Bansal, M. C. Valenti and J. B. Kenney. Adaptive content control for communication amongst cooperative automated vehicles. Presented at Wireless Vehicular Communications (WiVeC), 2014 IEEE 6th International Symposium On. 2014.

[114] IEEE Std 802.11p-2010, "IEEE Standard for Information technology, Telecommunications and information exchange between systems, Local and metropolitan area networks, Specific requirments, Part 11: Wireless LAN Medium and Access Control (MAC) and Physical Layer (PHY) Specifications, Amendment 6: Wireless Access in Vehicular Environments," .

[115] C. Sommer, S. Joerer and F. Dressler. On the applicability of two-ray path loss models for vehicular network simulation. Presented at Vehicular Networking Conference (VNC), 2012 IEEE. 2012.

[116] T. S. Rappaport, Wireless Communications: Principles and Practice. Upper Saddle River, New Jersey: Prentice Hall PTR, 2009.

[117] E. W. Stacy, "A Generalization of the Gamma Distribution," Ann. Math. Statist., vol. 33, pp. 1187-1192, 1962. 
[118] M. D. Yacoub. The $\alpha-\mu$ distribution: A general fading distribution. Presented at Personal, Indoor and Mobile Radio Communications, 2002. the 13th IEEE International Symposium On. 2002.

[119] M. D. Yacoub. The $\alpha-\mu$ distribution: A physical fading model for the stacy distribution. Vehicular Technology, IEEE Transactions On 56(1), pp. 27-34. 2007.

[120] J. Reig and L. Rubio. Estimation of the composite fast fading and shadowing distribution using the logmoments in wireless communications. Wireless Communications, IEEE Transactions On 12(8), pp. 3672-3681. 2013.

[121] S. Kokalj-Filipovic, L. Greenstein, B. Cheng and M. Gruteser, "Methods for extracting V2V propagation models from imperfect RSSI field data," in IEEE 82nd Vehicular Technology Conference (VTC), 2015.

[122] O. Gomes, C. Combes and A. Dussauchoy. Parameter estimation of the generalized gamma distribution. Math. Comput. Simul. 79(4), pp. 955-963. 2008.

[123] J. Reig and L. Rubio. On simple estimators of the $\alpha-\mu$ fading distribution. Communications, IEEE Transactions On 59(12), pp. 3254-3258. 2011.

[124] E. W. Stacy and G. A. Mihram. Parameter estimation for a generalized gamma distribution. Technometrics 7(3), pp. 349-358. 1965.

[125] F. Bouchereau and D. Brady. Method-of-moments parameter estimation for compound fading processes. Communications, IEEE Transactions On 56(2), pp. 166-172. 2008.

[126] A. Dogandzic and Jinghua Jin. Maximum likelihood estimation of statistical properties of composite gammalognormal fading channels. Signal Processing, IEEE Transactions On 52(10), pp. 2940-2945. 2004.

[127] M. Abramowitz and I. A. Stegun, Handbook of Mathematical Functions: With Formulas, Graphs, and Mathematical Tables. Dover Books on Mathematics, 1972.

[128] A. Papoulis and S. U. Pillai, Probability, Random Variables and Stochastic Processes. McGraw-Hill, 2002.

[129] K. D. Kim and P. R. Kumar. Cyber-Physical systems: A perspective at the centennial. Proceedings of the IEEE 100(Special Centennial Issue), pp. 1287-1308. 2012.

[130] P. Derler, E. A. Lee and A. Sangiovanni Vincentelli. Modeling Cyber-Physical systems. Proceedings of the IEEE 100(1), pp. 13-28. 2012.

[131] E. A. Lee. Cyber physical systems: Design challenges. Presented at 2008 11th IEEE International Symposium on Object and Component-Oriented Real-Time Distributed Computing (ISORC). 2008.

[132] O. Maler, Z. Manna and A. Pnueli. From timed to hybrid systems. Presented at Real-Time: Theory in Practice. 1991.

[133] J. Lygeros, C. Tomlin and S. Sastry. Hybrid systems: Modeling, analysis and control. Preprint 1999.

[134] S. Rezaei and R. Sengupta. Kalman filter-based integration of DGPS and vehicle sensors for localization. IEEE Transactions on Control Systems Technology 15(6), pp. 1080-1088. 2007.

[135] D. Rossi, R. Fracchia and M. Meo. VANETs: Why use beaconing at all? Presented at IEEE International Conference on Communications, 2008.

[136] J. Mittag, F. Thomas, J. Harri and H. Hartenstein. A comparison of single- and multi-hop beaconing in VANETs. Presented at Proceedings of the Sixth ACM International Workshop on VehiculAr InterNETworking. 2009.

[137] V. Kulathumani, R. A. Moparthi and Y. P. Fallah, "VCAST: Scalable Dissemination of Vehicular Information with Distance-Sensitive Precision," International Journal of Distributed Sensor Networks, vol. 2013, 2013.

[138] S. Ni, Y. Tseng, Y. Chen and J. Sheu. The broadcast storm problem in a mobile ad hoc network. Presented at Proceedings of the 5th Annual ACM/IEEE International Conference on Mobile Computing and Networking. 1999.

[139] S. Yousefi, E. Altman, R. El-Azouzi and M. Fathy. Analytical model for connectivity in vehicular ad hoc networks. IEEE Transactions on Vehicular Technology, 57(6), pp. 3341-3356. 2008.

[140] NHTSA, "Analysis of pedestrian crashes," Tech. Rep. DOT HS 809 585, April 2003.

[141] NHTSA, "Pedestrians, traffic safety facts," Tech. Rep. DOT HS 811 748, August 2013.

[142] S. B. McLaughlin, J. M. Hankey, T. A. Dingus and S. G. Klauer, "Development of an FCW algorithm evaluation methodology with evaluation of three alert algorithms," Tech. Rep. DOT HS 811 145, June 2009.

[143] A. Kanaris, E. B. Kosmatopoulos and P. A. Loannou. Strategies and spacing requirements for lane changing and merging in automated highway systems. IEEE Transactions on Vehicular Technology 50(6), pp. 1568-1581. 2001.

[144] B. K. Chaurasia and S. Verma. Haste induced behavior and VANET communication. Presented at Vehicular Electronics and Safety (ICVES), 2009 IEEE International Conference On. 2009.

[145] D. Caveney. Cooperative vehicular safety applications. IEEE Control Systems 30(4), pp. 38-53. 2010. 
[146] P. Auer, H. Burgsteiner and W. Maass. A learning rule for very simple universal approximators consisting of a single layer of perceptrons. Neural Networks 21(5), pp. 786-795. 2008.

[147] G. Guo and W. Yue. Sampled-data cooperative adaptive cruise control of vehicles with sensor failures. IEEE Transactions on Intelligent Transportation Systems 15(6), pp. 2404-2418. 2014.

[148] NHTSA, "Safety pilot model deployment," Tech. Rep. DOT HS 812 171, June 2015. 2015.

[149] J. Laumond, S. Sekhavat and F. Lamiraux. "Guidelines in nonholonomic motion planning for mobile robots," in Robot Motion Planning and ControlAnonymous 1998, . 